\title{
FEYNMAN AMPLITUDES, COACTION PRINCIPLE, AND COSMIC GALOIS GROUP
}

\author{
FRANCIS BROWN
}

ABstract. The first part of a set of notes based on lectures given at the IHES in May 2015 on Feynman amplitudes and motivic periods.

0.1. Some motivation for physicists. Scattering amplitudes are ubiquitous in high energy physics and have been intensively studied from at least three angles:

(1) in phenomenology, where amplitudes in quantum field theory are obtained as a sum of Feynman integrals associated to graphs which represent interactions between fundamental particles. This presents a huge computational challenge with important applications to collider experiments.

(2) in superstring perturbation theory, where amplitudes are expressed as integrals over moduli spaces of curves with marked points.

(3) in various modern approaches, most notably in the planar limit of $N=$ 4 SYM, which avoid the use of Feynman graphs altogether and seek to construct the amplitude directly, either via the bootstrap method, or via geometric approaches such as on-shell diagrams or the amplituhedron.

The goal of these notes is to study a new kind of structure which is potentially satisfied by amplitudes in all three situations. To motivate it, consider first the case of the dilogarithm function, defined for $|z|<1$ by the sum

$$
\operatorname{Li}_{2}(z)=\sum_{n \geq 1} \frac{z^{n}}{n^{2}} .
$$

It is an iterated integral over the projective line minus three points, and is the universal function describing amplitudes at one loop. Chen's general theory of iterated integrals [26] naturally associates to it a coproduct $\Delta^{\text {it }}$ satisfying

$$
\Delta^{\mathrm{it}} \mathrm{Li}_{2}(z)=1 \otimes \mathrm{Li}_{2}(z)+\mathrm{Li}_{1}(z) \otimes \log (z)+\mathrm{Li}_{2}(z) \otimes 1
$$

where $\operatorname{Li}_{1}(z)=-\log (1-z)$. Suitably interpreted, this coproduct encodes both the differential equation $\frac{\partial}{\partial z} \operatorname{Li}_{2}(z)=\operatorname{Li}_{1}(z) d \log (z)$, and also the action of monodromy $\mathrm{Li}_{2}(z) \mapsto \mathrm{Li}_{2}(z)+2 \pi i \log (z)$ as $z$ winds around the point $z=1$ in the positive direction. It is well-known that Feynman integrals and amplitudes of different orders can be related both with respect to differentiation, and also with respect to branch cuts, and so it comes as no surprise that the coproduct $\Delta^{\text {it }}$ has found many uses in high-energy physics via the so-called method of symbols [36].

Now consider the much more subtle situation when $z=1$. Then $\operatorname{Li}_{2}(1)=\zeta(2)$ is simply a number and all the structure described above seems to be lost. It can, however, be retrieved by replacing $\zeta(2)$ with a 'motivic period' $\zeta^{\mathfrak{m}}(2)$, which as a first approximation, can be simply thought of as a matrix of numbers (in this case, 
a two by two matrix). It now satisfies a coaction, rather than a coproduct:

$$
\Delta \zeta^{\mathfrak{m}}(2)=\zeta^{\mathfrak{m}}(2) \otimes 1
$$

The dilogarithm can also be promoted a motivic version $\mathrm{Li}_{2}^{\mathfrak{m}}(z)$ in a similar manner, and has a (unipotent) coaction:

$$
\Delta \mathrm{Li}_{2}^{\mathfrak{m}}(z)=\mathrm{Li}_{2}^{\mathfrak{m}}(z) \otimes 1+\mathrm{Li}_{1}^{\mathfrak{m}}(z) \otimes \log ^{\mathfrak{u}}(z)+1 \otimes \operatorname{Li}_{2}^{\mathfrak{u}}(z)
$$

which is valid both for $z$ viewed as a function, and also for any algebraic values of $z$, including $z=1$ (in which case, $\operatorname{Li}_{2}^{\mathfrak{m}}(1)=\zeta^{\mathfrak{m}}(2)$ and $\log ^{\mathfrak{u}}(1)=\operatorname{Li}_{2}^{\mathfrak{u}}(1)=0$ ). The quantities on the right-hand side of the tensor product are of a different nature from those on the left, and could be called unipotent de Rham periods. This coaction is a much deeper structure than the coproduct $\Delta^{\text {it }}$. Motivic periods have a natural homomorphism per (called the 'period map') to the complex numbers: for example, $\operatorname{per}\left(\operatorname{Li}_{2}^{\mathfrak{m}}(z)\right)=\operatorname{Li}_{2}(z)$.

If we imagine that Feynman integrals and scattering amplitudes more generally have canonical 'motivic' versions, as one certainly expects, then they inherit a coaction, and it is natural to ask how this coaction relates to the structure of amplitudes. Indeed, any of the three situations described above should generate a space $H$ of motivic periods, and a corresponding algebra $A$ of de Rham periods. A general 'coaction principle' would be the equation

$$
\Delta H \subset H \otimes A \text {. }
$$

In other words, the class of amplitudes is stable under the coaction. An equivalent way to phrase this is in terms of group theory. Indeed, $A$ is naturally a Hopf algebra, and defines a pro-algebraic group $C$, a projective limit of algebraic matrix groups, whose elements are homomorphisms from $A$ to a commutative ring. The equation (0.1) is equivalent to a linear group action of $C$ on $H$ :

$$
C \times H \longrightarrow H \text {. }
$$

In other words, the space of amplitudes in the theory are stable under the action of a group, which could be called a 'cosmic' Galois group, to borrow a phrase from Cartier [24].

What possible evidence is there for such a structure? Taking each of the three situations in turn, we find the following:

(1) In quantum field theory, Panzer and Schnetz computed every known amplitude in massless $\phi^{4}$ theory, and, assuming the 'period conjecture' replaced them with their motivic versions [15], generating an algebra $H$. Remarkably, they found that the coaction principle (0.1) holds in every case. Evidence in $[1,2]$ suggests that Feynman amplitudes of small graphs with non-trivial masses and momenta also satisfy a similar property.

(2) In string perturbation theory, Stieberger and Schlotterer [47] replaced the multiple zeta values in the expansion of the genus zero open string with their motivic versions [15]. They found that the coaction gives rise to a spectacular factorisation of the amplitude, which is invisible on the level of numbers. Similarly, the closed genus zero string can be expressed in terms of single-valued multiple zeta values [53], whose motivic versions are also known to be closed under the coaction (0.1).

(3) Several features of the hexagon function bootstrap [25] are expressible in terms of a coaction principle (0.1). Here, $H$ is a subspace of the space of 
motivic iterated integrals on the moduli space of curves $\mathcal{M}_{0,6}$ of genus 0 with six marked points [17] $\$ 10.6 .2$, and equation (0.1) is equivalent to 'first $n$ entries' constraints on the iterated integrals.

In all these settings, we believe that a version of the coaction principle (0.1) holds, after possibly enlarging the space of integrals or amplitudes under consideration. It is also important to note that in the first setting, the coaction principle holds graph by graph, whereas in the third setting, it operates on the entire amplitude, i.e., the sum of all graphs (these two statements are by no means equivalent). It would be interesting in (1) to study the case of gauge theories: the (three) known coefficients of the perturbative expansion of the anomalous magnetic dipole moment of the electron also appear to exhibit a coaction principle.

In this paper, we concentrate only on the first setting. Our first goal, then, is to define canonical motivic Feynman integrals for a large class of graphs in perturbative quantum field theory in an even number of space-time dimensions. We then develop tools to prove that, after enlarging the space of motivic integrals under consideration slightly, the coaction principle (0.1) does indeed hold. This is just the first step in a programme to study amplitudes via the representation theory of groups.

The accompanying paper [17] develops methods for studying motivic periods including a decomposition map which generalises the $f$-alphabet construction used in [47] for multiple zeta values, and general notions of symbols which go beyond the polylogarithmic setting. In particular, it provides tools to compare amplitudes at different weights via seemingly paradoxical operations such as 'differentiation with respect to $\zeta(3)^{\prime}$, and to extract the essential information contained in an amplitude in a basis-independent manner.

0.2. Statement of results. To any Feynman graph $G$ one associates a Feynman integral, which is given by a possibly divergent projective integral of the general parametric form

$$
I_{G}(q, m)=\int_{\sigma_{G}} \omega_{G} \quad \text { where } \quad \omega_{G}=\frac{P\left(\alpha_{e}\right) \Omega_{G}}{\Psi_{G}^{A} \Xi_{G}(q, m)^{B}} .
$$

Here $\Psi_{G}, \Xi_{G}(q, m)$, and $P\left(\alpha_{e}\right)$ are certain polynomials in variables $\alpha_{e}$ indexed by the edges of $G, \Omega_{G}$ is defined in (1.12), $A, B \in \mathbb{Z}$, and $\sigma_{G}$ is the domain where all $\alpha_{e} \geq 0$. These quantities are involved in predictions for particle collider experiments. The polynomial $P$ allows for the possibility of numerator polynomials in the loop momentum representation, and therefore covers the case of Feynman integrals in gauge theories (before renormalisation). It is immediate from the integral representation (0.3), when it converges, that amplitudes are families of periods [40], depending on kinematic data such as particle momenta $q=\left(q_{i}\right)_{i}$ and masses $m=\left(m_{e}\right)_{e}$. A deep idea, originating with Grothendieck, is that there should exist a Galois theory of periods [5, 6], extending the classical Galois theory of algebraic numbers. We shall apply these ideas to the integrals of the form (0.3).

The first problem, when trying to set up a Galois theory of periods, is that one immediately runs into difficult conjectures concerning motives. A simple way around this is to work in a category of systems of realisations ${ }^{1}$ and the second part of these notes [17] explain how this can be done without difficulty. In brief, the objects in a category of realisations $\mathcal{H}(S)$ on a smooth scheme $S$ over $\mathbb{Q}$ are

\footnotetext{
${ }^{1}$ We shall abusively use the word 'motive' to signify an object in such a category which is the image of the cohomology of an algebraic variety.
} 
triples $\mathcal{V}=\left(\mathcal{V}_{B}, \mathcal{V}_{d R}, c\right)$ where $\mathcal{V}_{B}$ is a local system of $\mathbb{Q}$-vector spaces over $S(\mathbb{C})$; $\mathcal{V}_{d R}$ is an algebraic vector bundle with integrable connection on $S$ and regular singularities at infinity, and $c$ is a Riemann-Hilbert correspondence between $\mathcal{V}_{B}$ and $\mathcal{V}_{d R}$. This data should define a variation of mixed Hodge structures on $S$. A further subtlety, which has nothing to do with questions about motives, is how to interpret any given family of integrals, such as (0.3), as a period of the cohomology of an algebraic variety since this can involve choices. It turns out that it can be done canonically for Feynman amplitudes.

Theorem 0.1. For any Feynman graph $G$ with generic kinematics $q, m$, there is a canonical way to associate to a convergent integral (0.3):

(i). an object $\operatorname{mot}_{G}$ in $\mathcal{H}(S)$, where $S$ is a Zariski open in a space of kinematics,

(ii). a de Rham class $\left[\omega_{G}\right]$ in the generic fiber of $\left(\operatorname{mot}_{G}\right)_{d R}$,

(iii). a Betti class $\left[\sigma_{G}\right]$ in a certain (Euclidean) fiber of $\left(\operatorname{mot}_{G}\right)_{B}^{\vee}$, such that the integral (0.3) is the period

$$
\sigma_{G}\left(c\left(\omega_{G}\right)\right)=I_{G}(q, m) .
$$

The object $\operatorname{mot}_{G}$ is defined as a compatible system of cohomology groups (namely, Betti and de Rham) of a family of pairs of algebraic varieties

$$
\left(\mathbb{P}^{G} \backslash Y_{G}, D \backslash\left(D \cap Y_{G}\right)\right)
$$

where $\mathbb{P}^{G}$ is a blow up of projective space along linear subvarieties, $Y_{G}$ is the strict transform of the zero loci of $\Psi_{G}$ and $\Xi_{G}(q, m)$, and $D$ is a certain strict normal crossing divisor independent of $q, m$. This theorem generalises a result in $[10]$ which treats a family of cases with no kinematic dependence $(B=0$ and $S$ is a point).

This theorem enables us to replace the Feynman integral $I_{G}(q, m)$ with a canonical 'motivic version' $I_{G}^{\mathfrak{m}}(q, m)=\left[\operatorname{mot}(G), \sigma_{G}, \omega_{G}\right]^{\mathfrak{m}}$ which is defined as a matrix coefficient of the torsor of isomorphisms between two fiber functors on $\mathcal{H}(S)$. The integral itself (0.3) can be retrieved from it by applying the period homomorphism. The motivic integral now carries the action of an affine group scheme which is the Tannaka group of $\mathcal{H}(S)$ with respect to the de Rham fiber functor at the generic point. This group factors through a certain quotient which acts faithfully on the motivic periods of $\operatorname{mot}_{G}$ relative to $\sigma_{G}$, where $G$ has at most $Q$ external momenta and $M$ possible non-zero masses, hereafter called 'of type $(Q, M)$ '. This quotient is denoted by $C_{Q, M}$ and could be called a cosmic Galois group, following [24]. In this way, every convergent integral $(0.3)$ is replaced by a finite-dimensional representation of $C_{Q, M}$, and this enables us to assign an array of new invariants to amplitudes. Examples include: a weight filtration ${ }^{2}$, a canonical connection (differential equation with regular singularities), Hodge polynomials, Galois conjugates, and various measures of complexity such as the rank, dimension, and unipotency degree. These ideas are explored in the second half of these notes [17]. In particular, we generalise the notion of the ' $f$-alphabet' decomposition of multiple zeta values, which has various applications in physics, to all periods. The next step in this programme is to formulate conjectures which relate topological invariants of graphs to the above-mentioned invariants of their motivic periods.

\footnotetext{
${ }^{2}$ This provides a rigorous meaning to many statements in the physics literature referring to the 'transcendental weight' of amplitudes
} 
We can subsequently define $\mathcal{F} \mathcal{P}_{Q, M}^{\mathfrak{m}}$ to be the vector space spanned by all motivic periods of motic ${ }^{3}$ Feynman graphs of type $(Q, M)$ relative to $\sigma_{G}$. The group action

$$
C_{Q, M} \times \mathcal{F} \mathcal{P}_{Q, M}^{\mathfrak{m}} \longrightarrow \mathcal{F P}_{Q, M}^{\mathfrak{m}}
$$

can be expressed equivalently as a coaction

$$
\Delta: \mathcal{F} \mathcal{P}_{Q, M}^{\mathfrak{m}} \longrightarrow \mathcal{F P}_{Q, M}^{\mathfrak{m}} \otimes_{k_{Q, M}} \mathcal{F P}_{Q, M}^{\mathfrak{d r}}
$$

where $\mathcal{F} \mathcal{P}_{Q, M}^{\mathfrak{d r}}$ is the ring generated by the de Rham periods of $\operatorname{mot}_{G}$. Note that the formula we gave for the motivic dilogarithm in the first paragarph involved the unipotent coaction and unipotent de Rham periods for simplicity (the full coaction involves powers of $\mathbb{L}^{\mathfrak{d r}}$, see [17], last lines of $\left.\$ 10\right)$. A key point is that there is a general formula for this coaction in terms of the cohomology $\left(\operatorname{mot}_{G}\right)_{d R}$, and this can be computed explicitly in some cases. The apparently unphysical case of graphs with no masses or momenta plays a special role in this theory. Indeed, $\mathcal{F} \mathcal{P}_{0,0}^{\mathfrak{m}}$ is an algebra, and each $\mathcal{F P}_{Q, M}^{\mathfrak{m}}$ is a module over it:

$$
\mathcal{F} \mathcal{P}_{0,0}^{\mathfrak{m}} \times \mathcal{F} \mathcal{P}_{Q, M}^{\mathfrak{m}} \longrightarrow \mathcal{F} \mathcal{P}_{Q, M}^{\mathfrak{m}} .
$$

0.3. Product structure and stability. There is a priori no reason whatsoever for the action of $C_{Q, M}$ to preserve the space of motivic amplitudes, which form a small subfamily of integrals (0.3) with highly specific numerators. ${ }^{4}$ However, using the formalism of motivic periods, Panzer and Schnetz computed the Galois action on a huge family of amplitudes in $\phi^{4}$ theory of type $(0,0)$ and verified, astonishingly, that they are preserved by $C_{0,0}$ in all cases. The motivation for these lectures was to try to understand this extraordinary fact.

The theory outlined in these notes is best explained by the following conjecture.

Conjecture 1. The motivic periods of a graph $G$ of type $(Q, M)$ are regularised versions of motivic integrals of the form (0.3). Those of weight $\leq k$ are in the algebra generated by regularised motivic periods of graph minors of $G$ with at most $k+1$ edges.

This conjecture means that the Galois conjugates of a motivic amplitude of low weight of a graph should be a regularised motivic period of its sub-quotient graphs of the form (0.3). Since there are few graphs with a bounded number of edges, this provides a constraint on amplitudes to all orders. We call this the small graphs principle. The upshot is that the topology of a graph constrains the Galois theory of its amplitudes. In the case $(Q, M)=(0,0)$, this theory partially explains the observations of Panzer and Schnetz.

What is presently lacking for a proof of this conjecture is a suitable notion of regularisation for motivic periods. ${ }^{5}$ In these notes, we prove a weaker, but more

\footnotetext{
${ }^{3}$ This notion is defined in $\S 3$ and generalises the notion of one-particle irreducible.

${ }^{4}$ From now on, we shall loosely call amplitude a Feynman integral of the form (0.3) with a specific numerator, which arises, for instance, from the Feynman rules of a given quantum field theory, as opposed to an arbitrary integral with that shape. In the literature, the word amplitude is often reserved to describe the sum of all Feynman integrals at a given loop order. We shall call the latter the 'full amplitude' in accordance with some authors.

${ }^{5}$ Unfortunately, when writing the technical background [17] for these notes, I had not forseen that convergent Feynman integrals might require the theory of limiting mixed Hodge structures, and a corresponding notion of limiting motivic period, and so it was not discussed. I do not believe that this, or conjecture 1 , should pose any major difficulties.
} 
precise version of this conjecture, in which we replace the word 'regularised' with 'affine', which has a technical meaning (theorem 8.11). It implies the

Theorem 0.2. The vector space $W_{k} \mathcal{F P}_{Q, M}^{\mathfrak{m}}$ is finite-dimensional. In particular, the vector space generated by convergent integrals (0.3) which are of bounded weight ${ }^{6}$, for $G$ of any fixed type $(Q, M)$, is finite-dimensional.

This theorem is non-trivial since there are infinitely many graphs, and therefore implies infinitely many relations between periods of different graphs.

We also show that the weight-graded pieces of $\operatorname{mot}(G)$ in weight $\leq k$ are indeed generated by minors of $G$ with at most $\leq k+1$ edges, and we completely determine $W_{2} \mathcal{F} \mathcal{P}_{0,0}^{\mathfrak{m}}$. These simple facts already imply strong and concrete constraints on amplitudes to all loop orders - for example, the last statement gives a non-trivial and rather subtle condition on amplitudes which has been verified numerically for nine loop scalar graphs by Panzer and Schnetz.

At the heart of this theory is a set of identities for graph polynomials. For the usual graph (Kirchhoff) polynomial it takes the form of a partial factorisation

$$
\Psi_{G}=\Psi_{\gamma} \Psi_{G / \gamma}+R_{\gamma, G}^{\Psi}
$$

where $\gamma \subset G$ is any subgraph (defined by a subset of edges of $G$ ), and $R_{\gamma, G}^{\Psi}$ is a remainder term of higher degree in the variables of $\Psi_{\gamma}$ than $\operatorname{deg}\left(\Psi_{\gamma}\right)$. This identity has been known for some time and is used in the parametric theory of renormalisation [21], although only in the special case when $\gamma$ is a divergent subgraph. It generalises in two different ways for the graph polynomial $\Xi_{G}(q, m)$, which we call the ultraviolet and infra-red factorisations. This requires some genericity assumptions on the external kinematics. The infra-red factorisation identities are new.

The geometric incarnation of these identities implies that the open strata of $(0.4)$

$$
D_{i} \backslash\left(Y_{G} \cap D_{i}\right)
$$

are products of varieties of the same type. This was already observed in [10] in the case $(Q, M)=(0,0)$ mentioned above. Such a family of varieties defines a type of operad in the category of schemes over $\mathbb{Z}$. Although we shall barely mention operads in these notes, the notion of 'operad in the category of motives' imposes strong constraints on its possible periods, and merits further study. ${ }^{7}$ A similar theory to the one described here should hold more generally for any family of varieties with this product-structure.

We are still very far from exploiting all the consequences of this geometric structure underlying amplitudes. In fact, the product structure on the faces of $(0.4)$ is such a rigid constraint that it almost completely determines the polynomials $\Psi_{G}$ and $\Xi_{G}(q, m)$, as we prove in $\S 4$.

0.4. Contents. In $\S 1$ we recall some basic notions relating to Feynman graphs and graph polynomials. In $\S 2$ we prove factorisation theorems for graph polynomials. In $\S 3$ we study the notion of a motic subgraph of a Feynman graph. These are in oneto-one correspondence with the locus where the domain of integration $\sigma_{G}$ meets the singularities of the Feynman integrand and subsume both ultraviolet and infra-red

\footnotetext{
${ }^{6}$ let us call a period of weight $\leq k$ if it is the image of a motivic period of weight $\leq k$ under the period homomorphism

${ }^{7}$ A similar example of such a system of stratified varieties with product structure are the moduli spaces $\mathfrak{M}_{g, n}$ of curves of genus $g$ with $n$ marked points.
} 
type divergences. In $\S 4$ we consider a Hopf algebra of graphs where the coproduct ranges over motic subgraphs and characterize graph polynomials by their partial factorisation properties. The motic Hopf algebra is a generalisation of the ConnesKreimer coproduct for scalar graphs since it also takes into account certain infra-red subdivergences. In $\S 5$ we study blow-ups of projective space along linear subspaces, and define some affine models which generalise the partial compactifications of moduli spaces $\mathfrak{M}_{0, n}^{\delta}$ of the author's thesis. In $\S 6$ we define the graph motive and prove its recursive product structure. In $\S 7$ we define the motivic amplitude of a Feynman graph, and prove some stability results in $\S 8$. In $\S 9$ we focus on the case of graphs with no kinematic dependence and prove some modest results in the direction of the coaction conjecture of [46]. Although they are of limited physical significance, this family of graphs plays an important and central role in the theory. In $\S 10$ we discuss applications of the cosmic Galois group in the case of graphs with general kinematics and state some conjectures and problems for further study.

The appendix $\S 12$ provides a fully worked example of such a graph using the methods described here. It requires some technical tools which are set out in $\S 11$.

0.5. Relation to other work. A number of expressions in this paper have appeared in the literature with possibly different meanings. They are listed below:

- 'Cosmic Galois group'. The phrase 'cosmic Galois group' was invented by P. Cartier. Later, Connes and Marcolli made a precise definition of a cosmic Galois group in relation to renormalisation, in the papers [27, 28]. It is not clear if it is at all related to the groups defined here.

- 'Motivic amplitude'. This phrase occurs with a different definition in [34], where it means a certain tensor of elements in a field, and only makes sense in the mixed Tate case. This notion can be retrieved as a very special case of the symbol (defined in [17]) of de Rham motivic amplitudes.

- 'Motivic multiple zeta value'. There are two versions of motivic multiple zeta values in use, an earlier one due to Goncharov, for which the motivic version of $\zeta(2)$ vanishes, and which do not posses a period map, and another for which $\zeta^{\mathfrak{m}}(2)$ is non-zero. Sending $\zeta^{\mathfrak{m}}(2)$ to zero would destroy much of the structure in amplitudes discovered in [46].

It is also important to emphasize that motivic amplitudes do not form a Hopf algebra. The main coaction formula (0.6) is asymmetric - on one side we have motivic periods which have a well-defined map to numbers, and on the other, de Rham periods which do not. However we can associate symbols to de Rham periods (in the differentially unipotent case), and also single-valued periods [17].

0.6. Acknowledgements. This work was partly funded by ERC grant 257638. I owe many thanks to the participants of my lecture series at IHES for their interest and many pertinent questions, especially Pierre Cartier and Joseph Oesterlé. Many thanks to Oliver Schnetz and Erik Panzer for their remarkable computations, which motivated this project, and also to Clément Dupont and Lance Dixon for valuable feedback.

\section{Scalar Feynman GRAPHS AND SymanZIK POLYNOMials}

We first recall some basic definitions of scalar Feynman graphs, before describing the corresponding integrals in parametric form. The reader should be aware that our conventions occasionally differ from the standard ones in a few minor details. 
1.1. Feynman graphs. A Feynman graph is a graph $G$ defined by

$$
\left(V_{G}, E_{G}, E_{G}^{\mathrm{ext}}\right)
$$

where $V_{G}$ is the set of vertices of $G, E_{G}$ is the set of internal edges of $G$, and $E^{\text {ext }}$ is a set of external half-edges (also known as legs). Their endpoints are encoded by maps $\partial: E_{G} \rightarrow \operatorname{Sym}^{2} V_{G}$ and $\partial: E_{G}^{\text {ext }} \rightarrow V_{G}$. We shall assume that the vertices with external legs (image of $E_{G}^{\text {ext }}$ in $V_{G}$ ) lie in a single connected component of $G$.

A Feynman graph additionally comes with kinematic data:

- a particle mass $m_{e} \in \mathbb{R}$ for every internal edge $e \in E_{G}$,

- a momentum $q_{i} \in \mathbb{R}^{d}$ for every external half-edge $i \in E_{G}^{\text {ext }}$,

where $d \geq 0$, the dimension of space-time, is fixed. The internal edges of $G$ are labelled if there is a bijection $E_{G} \leftrightarrow S$ with a fixed set $S$. The external half-edges will be oriented inwards, so all momenta are incoming and are subject to momentum conservation

$$
\sum_{i \in E_{G}^{\mathrm{ext}}} q_{i}=0 .
$$

Some of the internal masses $m_{e}$ will be zero. Let $M_{G} \subset E_{G}$ denote the set of internal edges $e$ of $G$ for which $m_{e} \neq 0$. In our figures, the mass-carrying edges in $M_{G}$ will be drawn with a doubled edge.

In this paper, a subgraph $H$ of $G$ will be a graph defined by a triple $\left(V_{H}, E_{H}, E_{H}^{\text {ext }}\right)$ where $V_{H} \subset V_{G}, E_{H} \subset E_{G}$ and either $E_{H}^{\text {ext }}=E_{G}^{\text {ext }}$ or $E_{H}^{\text {ext }}=\emptyset$. Note that for $H$ to be a Feynman subgraph, the extra condition that the vertices $E_{H}^{\text {ext }}$ lie in a single connected component of $H$ must also hold. This guarantees that momentum conservation holds for every component of $H$. The particle masses of a Feynman subgraph $H \subset G$ are determined by the following condition:

$$
\begin{array}{lcll}
\text { either } & E_{H}^{\text {ext }}=E_{G}^{\text {ext }} & \text { and } & M_{H}=M_{G} \\
\text { or } & E_{H}^{\text {ext }}=\emptyset & \text { and } & M_{H}=\emptyset .
\end{array}
$$

In the former case, $H$ contains all massive edges of $G$, and inherits the corresponding masses. In the latter case, $H$ is viewed as a massless diagram and $m_{e}=0$ for all $e \in E_{H}$. Thus in these notes, a Feynman subgraph either meets all external legs in a single connected component, and contains all massive edges; or is considered to be massless with no external momenta.

Note that all external legs correspond to a potentially non-zero momentum; external legs which would ordinarily be considered to have zero incoming momentum will simply be omitted. This forces our graphs $G$ to have vertices of varying degrees: a graph $G$ is said to be in $\phi^{n}$ if every vertex has degree $\leq n$.

A tadpole, or self-edge, is a subgraph of $G$ of the form $(\{v\},\{v, v\}, \emptyset)$.

We shall use the following notation for the basic combinatorial invariants of $G$ :

$$
\begin{array}{ll}
h_{G}=h^{1}(G) & \text { the loop number of } G \\
\kappa_{G}=h^{0}(G) & \text { the number of connected components of } G \\
N_{G}=\left|E_{G}\right| & \text { the number of edges of } G .
\end{array}
$$

They do not depend on the external legs of $G$. Euler's formula states that

$$
N_{G}-V_{G}=h_{G}-\kappa_{G}
$$


We define the following equivalence relation on Feynman graphs. If a vertex $v \in V_{G}$ has several incoming momenta $q_{1}, \ldots, q_{n}$ we can replace it with a single incoming momentum $q_{1}+\ldots+q_{n}$ :
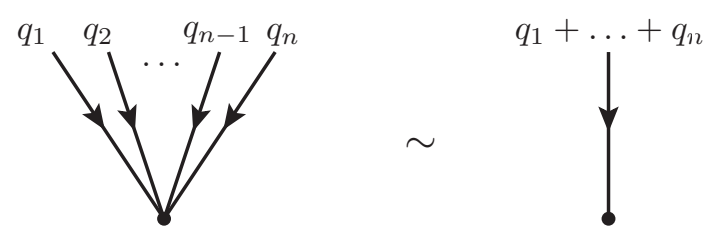

Our notion of Feynman subgraph respects this equivalence relation. The graph polynomials defined below will only depend on equivalence classes.

We say that a Feynman graph is of type $(Q, M)$ if it is equivalent to a graph with at most $Q$ external kinematic parameters, and at most $M$ non-zero particle masses. We shall call a graph one-particle irreducible, or 1PI, if every connected component is 2 -edge connected (i.e. deleting any edge causes the loop number to drop).

Example 1.1. The following Feynman graph will be our basic example to illustrate the ideas in this paper. It will be referred to several times throughout this text.

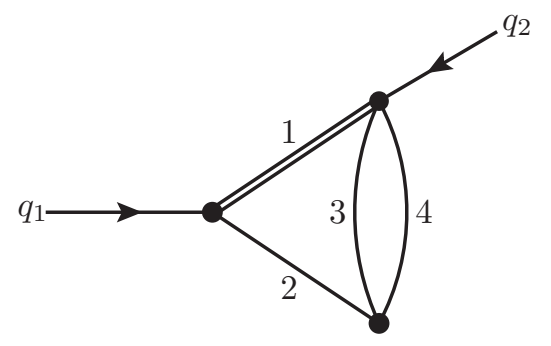

This graph has a single non-zero mass, namely $m_{1}$, and $m_{2}=m_{3}=m_{4}=0$. Momentum conservation demands that $q_{1}=-q_{2}$. The bottom right vertex, which meets edges $2,3,4$ has zero incoming external momentum.

1.2. Graph polynomials. Let $G$ be a Feynman graph. Recall that a tree is a connected graph $T$ with $h_{T}=0$. A forest is any graph $T$ with $h_{T}=0$.

Definition 1.2. A spanning $k$-tree of $G$ is a subgraph $T=T_{1} \cup \ldots \cup T_{k} \subset G$ which has exactly $k$ components $T_{i}$ such that $T_{i}$ is a tree and $V_{T}=V_{G}$.

A spanning 1-tree is simply called a spanning tree.

Definition 1.3. Let $G$ be a connected Feynman graph. The Kirchhoff polynomial (or 1st Symanzik polynomial) is the polynomial in $\mathbb{Z}\left[\alpha_{e}, e \in E_{G}\right]$ defined by

$$
\Psi_{G}=\sum_{T \subset G} \prod_{e \notin T} \alpha_{e}
$$

where the sum is over all spanning trees $T$ of $G$. If $G$ has several connected components $G_{1}, \ldots, G_{n}$ we shall define

$$
\Psi_{G}=\prod_{i=1}^{n} \Psi_{G_{i}}
$$


Note that one sometimes takes (1.3) as the general definition of graph polynomial. It differs from (1.4) and vanishes if $G$ has more than one connected component.

The second Symanzik polynomial is defined for connected $G$ by

$$
\Phi_{G}(q)=\sum_{T_{1} \cup T_{2} \subset G}\left(q^{T_{1}}\right)^{2} \prod_{e \notin T_{1} \cup T_{2}} \alpha_{e},
$$

where the sum is over all spanning 2 -trees $T=T_{1} \cup T_{2}$ of $G$ and $q^{T_{1}}=\sum_{i \in E_{T_{1}}^{\text {ext }}} q_{i}$ is the total momentum entering $T_{1}$. It equals $-q^{T_{2}}$ by momentum conservation (1.1). If $G$ has several connected components $G_{0}, G_{1}, \ldots, G_{n}$, then by our definition of a Feynman graph, exactly one component, say $G_{0}$, contains all external momenta.

In this case we define

$$
\Phi_{G}(q)=\Phi_{G_{0}}(q) \prod_{i \geq 1} \Psi_{G_{i}}
$$

Applying Euler's formula (1.2) to $G$ and a spanning $\kappa_{T}$-tree $T \subset G$ implies that

$$
N_{G}-N_{T}=h_{G}+\kappa_{T}-\kappa_{G} .
$$

Since the right-hand side is independent of $T, \Psi_{G}$ and $\Phi_{G}$ are homogeneous with respect to Schwinger parameters and have the following degrees (in the $\alpha_{e}$ ):

$$
\begin{aligned}
\operatorname{deg} \Psi_{G} & =h_{G} \\
\operatorname{deg} \Phi_{G}(q) & =h_{G}+1
\end{aligned}
$$

These equations can also be deduced from the contraction-deletion relations which are stated below. It is a crucial fact for the arithmetic of Feynman integrals that the coefficients of every monomial in $\Psi_{G}$ are only 0 or 1 . Furthermore, $\Psi_{G}$ and $\Phi_{G}$ are of degree at most one in every Schwinger parameter $\alpha_{e}$, but these two facts play a minor role in these notes. See [18], [22] for applications of these facts.

Definition 1.4. Let $G$ be a Feyman graph. Define

$$
\Xi_{G}(q, m)=\Phi_{G}(q)+\left(\sum_{e \in E_{G}} m_{e}^{2} \alpha_{e}\right) \Psi_{G}
$$

By (1.8), the polynomial $\Xi_{G}$ is homogeneous in the $\alpha_{e}$ of degree $h_{G}+1$.

Since the graph polynomials $\Psi_{G}, \Phi_{G}(q), \Xi_{G}(q, m)$ only depend on total momentum flow, they are well-defined on equivalence classes of graphs.

Example 1.5. Let $G$ be the graph of example 1.1. Then we have

$$
\begin{aligned}
\Psi_{G} & =\alpha_{1} \alpha_{3}+\alpha_{1} \alpha_{4}+\alpha_{2} \alpha_{3}+\alpha_{2} \alpha_{4}+\alpha_{3} \alpha_{4} \\
\Phi_{G}(q) & =q_{1}^{2}\left(\alpha_{1} \alpha_{2} \alpha_{3}+\alpha_{1} \alpha_{2} \alpha_{4}+\alpha_{1} \alpha_{3} \alpha_{4}\right) \\
\Xi_{G}(q, m) & =q_{1}^{2}\left(\alpha_{1} \alpha_{2} \alpha_{3}+\alpha_{1} \alpha_{2} \alpha_{4}+\alpha_{1} \alpha_{3} \alpha_{4}\right)+m_{1}^{2} \alpha_{1} \Psi_{G}
\end{aligned}
$$

Remark 1.6. The reader is warned that in the literature, the polynomial (1.9) is often written with the opposite sign (or equivalently, all momentum terms $q$ are replaced by $i q$ ). To find one's bearings, consider the following familiar bubble diagram with two equal masses $m_{1}=m_{2}=m$ : 


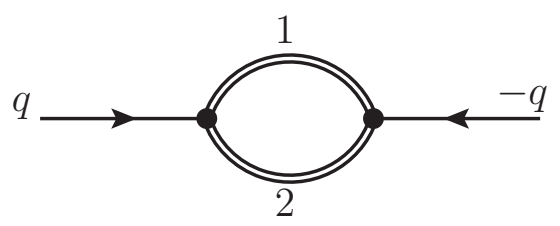

It satisfies

$$
\Xi_{G}(q, m)=q^{2} \alpha_{1} \alpha_{2}+m^{2}\left(\alpha_{1}+\alpha_{2}\right)^{2} .
$$

Its discriminant is $4 m^{2}+q^{2}$, and so its Landau singularity occurs at $q^{2}=-4 m^{2}$. With our chosen sign convention, it will lie outside the Euclidean region where $q, m$ are real. This, and other, typical physical infra-red singularities (where, for example, terms in $\Xi_{G}(q, m)$ cancel out altogether) will be excluded from the present set-up (although certain other types of infra-red singularities will be allowed). However, they can still be treated in the present theory on a graph by graph basis after analytic continuation in the space of kinematics $(q, m)$. That the analytic continuation exists follows from the fact that the discriminant locus is algebraic of codimension $\geq 1$, and its complement is connected in the analytic topology. Therefore there exists a path from a point in the Euclidean region to an open subset of the region $q \in i \mathbb{R}$, along which the integral is analytic.

1.3. Feynman integral in projective space. Let $d \in 2 \mathbb{N}$ be an even integer, which denotes the dimension of space-time. Here it will always be fixed, and will be dropped from the notation. Our version of the Feynman integral in parametric form differs marginally the usual presentation. In order to kill two birds (namely, the case with no kinematics, and the case with non-trivial kinematics) with one stone we shall take the following definition, after omitting certain pre-factors (see [21] $\S 3$ of a more rigorous derivation from first principles):

$$
I_{G}(q, m)=\int_{\sigma} \omega_{G}(q, m)
$$

where

$$
\omega_{G}(q, m)=\frac{1}{\Psi_{G}^{d / 2}}\left(\frac{\Psi_{G}}{\Xi_{G}(q, m)}\right)^{N_{G}-h_{G} d / 2} \Omega_{G}
$$

and

$$
\Omega_{G}=\sum_{i=1}^{N_{G}}(-1)^{i} \alpha_{i} d \alpha_{1} \wedge \ldots \wedge \widehat{d \alpha_{i}} \wedge \ldots \wedge d \alpha_{N_{G}}
$$

where $\widehat{d \alpha_{i}}$ means that the term $d \alpha_{i}$ is omitted. Note that the form $\omega_{G}$ is homogeneous of degree 0 , which follows from (1.8). Finally, let

$$
\sigma \subset \mathbb{P}^{N_{G}-1}(\mathbb{R})
$$

be the coordinate simplex defined in projective coordinates by

$$
\sigma=\left\{\left(\alpha_{1}: \ldots: \alpha_{N_{G}}\right) \in \mathbb{P}^{N_{G}-1}(\mathbb{R}): \alpha_{i} \geq 0\right\} .
$$

The integral (1.10) may not necessarily converge. Necessary and sufficient conditions for its convergence, in a certain kinematic region, will be stated below. The derivation of the parametric form of the Feynman integral (1.10) from its momentum space representation using the Schwinger trick is nicely explained in [44]. 
Remark 1.7. The integral (1.10) is a drastic simplification in certain situations. For example, if $d=4$ and $G$ is primitive overall log-divergent $\left(N_{G}=2 h_{G}\right)$, then the second factor in $\omega_{G}(q, m)$ drops out and it has no dependence on external masses or momenta. For such a graph, we obtain

$$
I_{G}=\int_{\sigma} \frac{\Omega_{G}}{\Psi_{G}^{2}} .
$$

In the case of the wheel with three spokes, this equals $6 \zeta(3)$ which is its residue (coefficient of $1 / \varepsilon$ in dimensional regularisation). The full vertex function is

$$
\int_{\sigma} \frac{\log \left(\Xi_{G}(q, m) / \Xi_{G}\left(q_{0}, m_{0}\right)\right)}{\Psi_{G}^{2}} \Omega_{G}
$$

where $q_{0}, m_{0}$ is a chosen renormalisation point. Such integrals, and their derivation, are discussed at length in [21]. They can also be viewed as period integrals either by writing the log in the numerator as an integral, or by differentiating with respect to a renormalisation scale as in [21] to make the integrand algebraic. It is highly likely that the theory described in this paper also extends to this situation, but a discussion of renormalisation would have made the present paper overly lengthy.

1.4. Edge subgraphs and their quotients. Let $G=\left(V_{G}, E_{G}, E_{G}^{\text {ext }}\right)$ be a Feynman graph. A set of internal edges $\gamma \subset E_{G}$ defines a subgraph of $G$ as follows. Write $E_{\gamma}=\gamma$ and let $V_{\gamma}$ be the set of endpoints of elements of $E_{\gamma}$.

Definition 1.8. A set of edges $\gamma \subset E_{G}$ is momentum-spanning if $\partial E_{G}^{\text {ext }} \subset V_{\gamma}$, and the vertices $E_{G}^{\text {ext }}$ lie in a single connected component of the graph $\left(V_{\gamma}, E_{\gamma}\right)$.

We define the subgraph associated to $\gamma \subset E_{G}$ by

$$
\left(V_{\gamma}, E_{\gamma}, E_{\gamma}^{\mathrm{ext}}\right)
$$

where $E_{\gamma}^{\text {ext }}=E_{G}^{\text {ext }}$ if $\gamma$ is momentum-spanning, and $E_{\gamma}^{\text {ext }}=\emptyset$ otherwise. Thus the Feynman graph (1.14) inherits all external momenta of $G$ if it is momentumspanning and has no external momenta otherwise. We shall call (1.14) the edgesubgraph associated to $\gamma$, and denote it also by $\gamma$ when no confusion arises.

The quotient of $G$ by an edge-subgraph $\gamma$ is defined by

$$
G / \gamma=\left(V_{G} / \sim,\left(E_{G} \backslash \gamma\right) / \sim, E_{G}^{\text {ext }} / \sim\right)
$$

where $\sim$ is the equivalence relation on vertices of $G$ where two vertices are equivalent if and only if they are vertices of the same connected component of $\gamma$, and the induced equivalence relation on edges (unordered pairs of vertices). It is a Feynman graph. Every connected component of $\gamma$ corresponds to a unique vertex in $G / \gamma$. Note that $\gamma$ is momentum-spanning if and only if $G / \gamma$ is equivalent to a graph with no external momenta (by momentum conservation). In this case we can take $E_{G / \gamma}^{\mathrm{ext}}=\emptyset$.

In this way, exactly one of the two Feynman graphs $\gamma$ and $G / \gamma$ is equivalent to a Feynman graph with non-zero external momenta: if $\gamma$ is momentum spanning it is $\gamma$, otherwise it is $G / \gamma$.

1.5. Contraction-deletion. Let $G=\left(V_{G}, E_{G}, E_{G}^{\text {ext }}\right)$ be a Feynman graph. The deletion of an edge $e$ in $G$ is the graph $G \backslash e$ defined by deleting the edge $e$ but retaining its endpoints:

$$
G \backslash e=\left(V_{G}, E_{G} \backslash\{e\}, E_{G}^{\mathrm{ext}}\right) .
$$


In general, it is not a union of Feynman graphs since momentum conservation may not hold on each of its connected components.

One sometimes encounters the following variant of the previous notion of graphquotient. It will be denoted by a double slash to distinguish it from the ordinary quotient. For an edge-subgraph $\gamma$, let $G / / \gamma$ be the empty graph if $h_{\gamma}>0$ and

$$
G / / \gamma=G / \gamma
$$

if $\gamma$ is a forest. In the case of a single edge $e, G / / e$ is empty whenever $e$ is a tadpole.

It follows from Euler's formula (1.2) that

$$
h_{G}=h_{\gamma}+h_{G / \gamma}
$$

for any edge-subgraph $\gamma \subset G$ (which is not necessarily connected).

Lemma 1.9. (Contraction-deletion) Let $G$ be connected, and $e \in E_{G}$. Then

$$
\begin{aligned}
\Psi_{G} & =\Psi_{G \backslash e}^{0} \alpha_{e}+\Psi_{G / / e} \\
\Phi_{G}(q) & =\Phi_{G \backslash e}^{0}(q) \alpha_{e}+\Phi_{G / / e}(q),
\end{aligned}
$$

where $\Psi_{G \backslash e}^{0}$ is given by the right-hand side of (1.3): it is $\Psi_{G \backslash e}$ if $G \backslash e$ is connected and 0 otherwise. Likewise $\Phi_{G \backslash e}^{0}(q)$ is given by the right-hand side of (1.5): it is equal to $\Phi_{G \backslash e}(q)$ if $G \backslash e$ is connected and equal to $\Psi_{G_{1}} \Psi_{G_{2}}\left(q^{G_{1}}\right)^{2}=\Psi_{G_{1}} \Psi_{G_{2}}\left(q^{G_{2}}\right)^{2}$ if $G \backslash e$ has two connected components $G_{1}, G_{2}$.

Proof. Let $T$ be a spanning $k$-tree of $G$ (where $k \in\{1,2\}$ ). The edge $e$ is not an edge of $T$ if and only if $T$ is a spanning $k$-tree of $G \backslash e$. By the definition of the graph polynomials, this gives rise to the first terms in the right hand sides of (1.16). Note that if $e$ is a tadpole, this is the only case which can occur. Now suppose that $e$ is not a tadpole. If $e$ is an edge of $T$, then $T / e$ is a spanning $k$-tree of $G / e$. Conversely, if $T^{\prime}$ is a spanning $k$-tree of $G / e$, then there is a unique component of $T^{\prime}$ which meets the vertex in $G / e$ defined by the endpoints of $e$. It follows that the inverse image of $T^{\prime}$ in $G$, together with the edge $e$, is a spanning $k$-tree in $G$. This establishes a bijection between the set of spanning $k$-trees in $T$ which contain $e$ and those of $G / e$. The rest follows from definition 1.3.

Corollary 1.10. It follows that from (1.16) and (1.9) that

$$
\begin{aligned}
\left.\Psi_{G}\right|_{\alpha_{e}=0} & =\Psi_{G / / e} \\
\left.\Xi_{G}(q, m)\right|_{\alpha_{e}=0} & =\Xi_{G / / e}(q, m) .
\end{aligned}
$$

1.6. Generic kinematics and non-vanishing of graph polynomials. We establish some non-vanishing results for graph polynomials which hold for generic momenta. These will be important for the sequel.

Lemma 1.11. A connected graph $G$ has a spanning tree. Equivalently, $\Psi_{G} \neq 0$.

Proof. Let $G$ be a connected graph with $\Psi_{G}=0$. Then $\Psi_{G / / e}=0$ for all $e$ by (1.16). By repeatedly contracting edges with distinct endpoints, we obtain a graph $G^{\prime}$ with a single vertex such that $\Psi_{G^{\prime}}=0$. It has a unique spanning tree consisting of this vertex, so $\Psi_{G^{\prime}}=\prod_{e \in E_{G^{\prime}}} \alpha_{e}$, which is non-zero, a contradiction.

Consider the following condition on external momenta

$$
\left(\sum_{i \in I} q_{i}\right)^{2} \neq 0 \quad \text { for all } I \subsetneq E_{G}^{\text {ext }} .
$$


It respects the equivalence relation of Feynman graphs.

Lemma 1.12. Let $G$ be a Feynman graph with non-trivial external momenta (in other words, there exists a vertex $v \in \partial E_{G}^{\text {ext }}$ such that the total momentum $q^{\{v\}}$ entering $v$ is non-zero). Then with condition $(1.17), \Phi_{G}(q) \neq 0$.

Proof. By momentum conservation (1.1), there exist at least two vertices $v_{1}, v_{2}$ with non-zero total incoming momenta $q_{1}, q_{2}$ respectively. Since $G$ is a Feynman graph, $v_{1}, v_{2}$ lie in the same connected component $G_{0}$. By the previous lemma, there exists a spanning tree $T$ in $G_{0}$. Since $T$ is connected, there is a (shortest) path from $v_{1}$ to $v_{2}$ contained in $T$. Delete any edge $e^{\prime}$ in this path to obtain a spanning 2 -tree $T \backslash e^{\prime}=T_{1} \cup T_{2}$ such that $v_{1} \in V_{T_{1}}$ and $v_{2} \in V_{T_{2}}$. It contributes a non-zero monomial $\left(q^{T_{1}}\right)^{2} \prod_{e \notin T_{1} \cup T_{2}} \alpha_{e}$ to the second Symanzik polynomial $\Phi_{G_{0}}(q)$ by (1.17). It cannot cancel out since all signs in the definition of $\Phi_{G_{0}}(q)$ are positive. Now apply (1.6) and the previous lemma to deduce that $\Phi_{G}(q) \neq 0$.

Now consider the following condition on momenta and masses:

$$
\left(\sum_{i \in I} q_{i}\right)^{2}+m_{e}^{2} \neq 0 \quad \text { for all } I \subsetneq E_{G}^{\text {ext }} \text { and } e \in E_{G} .
$$

Lemma 1.13. If (1.17) and (1.18) hold then $\Xi_{G}(q, m)=0$ if and only if $G$ has no massive edges, and no incoming momenta (i.e., $q^{\{v\}}=0$ for all $v \in \partial E_{G}^{\text {ext }}$ ).

Proof. Let $e \in E_{G}$ such that $m_{e} \neq 0$. Note that $\Phi_{G}(q)$ is of degree at most one in $\alpha_{e}$. If $G \backslash e$ is connected, then by (1.16), the coefficient of $\alpha_{e}^{2}$ in $\Xi_{G}$ is $m_{e}^{2} \Psi_{G \backslash e}$ which is non-zero by lemma 1.11. In particular, $\Xi_{G}(q, m) \neq 0$. In the opposite case, $e$ is a bridge in $G$, and $G \backslash e$ has two connected components $G_{1}, G_{2}$. Then $\Psi_{G}=\Psi_{G_{1}} \Psi_{G_{2}}$ and by (1.16) the coefficient of $\alpha_{e}$ in $\Xi_{G}(q, m)$ is $\left(\left(q^{G_{1}}\right)^{2}+m_{e}^{2}\right) \Psi_{G_{1}} \Psi_{G_{2}}$. This is non-zero by (1.18) and so $\Xi_{G}(q, m) \neq 0$. Finally, if all edges of $G$ are massless, then $\Xi_{G}(q, m)=\Phi_{G}(q)$ and we can appeal to the previous lemma.

Example 1.14. Consider the following Feynman graph

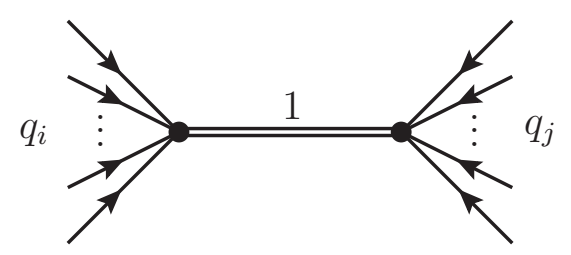

where the momenta entering on the left are $q_{i}$, for $i \in I$, and those on the right $q_{j}$, $j \in J$. Then $\Xi_{G}(q, m)=\left(\left(\sum_{i \in I} q_{i}\right)^{2}+m^{2}\right) \alpha_{1}$ is identically zero if $\left(\sum_{i \in I} q_{i}\right)^{2}+m^{2}=$ 0 , where $m=m_{1}$. These examples imply, by contraction and deletion, that the conditions (1.17) and (1.18) are optimal.

1.7. Space of generic kinematics. The previous discussion motivates the following definition. In order to allow the possibility of masses and momenta taking values in different fields, we work with affine spaces. Suppose that we wish to consider processes in $d \in 2 \mathbb{N}$ spacetime dimensions with $Q$ external momenta $\underline{q}=\left(q_{1}, \ldots, q_{Q}\right) \in \mathbb{A}^{Q d}$, which are subject to momentum conservation

$$
q_{1}+\ldots+q_{Q}=0,
$$


in a theory with $M$ possible non-zero particle masses $\underline{m}=\left(m_{1}, \ldots, m_{M}\right) \in \mathbb{G}_{m}^{M}$, where $\mathbb{G}_{m}=\mathbb{A}^{1} \backslash\{0\}$. The graph polynomials and hence the integral (1.10) are invariant under the action of the orthogonal group in $d$ dimensions. Therefore set

$$
s_{i, j}=s_{j, i}=q_{i} \cdot q_{j} \quad \text { for } 1 \leq i \leq j \leq Q
$$

to be the Euclidean inner product of the momenta $q_{i}, q_{j}$, and write

$$
s_{I}=\sum_{i, j \in I} s_{i, j}=\left(\sum_{i \in I} q_{i}\right)^{2} \quad \text { for } I \subset\{1, \ldots, Q\} .
$$

Condition (1.19) implies that the $s_{i, j} \in \mathbb{A}^{\left(\begin{array}{c}Q+1 \\ 2\end{array}\right)}$ lie in a subspace isomorphic to $\mathbb{A}^{\left(\begin{array}{l}Q \\ 2\end{array}\right)}$ since we can solve for $q_{Q}$. It is parameterised, for example, by the $s_{i, j}$ for $1 \leq i \leq j \leq Q-1$. The Feynman amplitude is a function only of the $s_{i, j}$ and $m_{k}$.

Definition 1.15. Define a space of generic kinematics

$$
K_{Q, M}^{\text {gen }} \subset K_{Q, M}=\mathbb{A}^{\left(\begin{array}{c}
Q \\
2
\end{array}\right)} \times \mathbb{G}_{m}^{M}
$$

with coordinates $(\underline{s}, \underline{m})$, to be the open complement of the union of the spaces

$$
s_{I}+m_{j}^{2}=0
$$

for all $I \subsetneq\{1, \ldots, Q\}$ and $j \in\{0,1, \ldots, M\}$, where we set $m_{0}=0$. Compare (1.17). It is an affine scheme defined over $\mathbb{Z}$ of dimension $M+\left(\begin{array}{c}Q \\ 2\end{array}\right)$. Define the Euclidean region to be its set of real points $K_{Q, M}^{\text {gen }}(\mathbb{R})$. Define a subspace

$$
U_{Q, M}^{\text {gen }} \subset K_{Q, M}^{\text {gen }}(\mathbb{C})
$$

to be the open region (in the usual topology) of $K_{Q, M}^{\text {gen }}(\mathbb{C})$ defined by

$$
\begin{aligned}
& U_{Q, M}^{\text {gen }}=\left\{(\underline{s}, \underline{m}) \in K_{Q, M}^{\text {gen }}(\mathbb{C}):\right. \operatorname{Re} s_{I}>0 \text { for all } I \subsetneq\{1, \ldots, Q\}, \\
&\text { and } \left.\quad \operatorname{Re} m_{j}^{2}>0 \text { for all } j \in\{1, \ldots, M\}\right\} .
\end{aligned}
$$

The region $U_{Q, M}^{\text {gen }}$ contains the Euclidean region $K_{Q, M}^{\text {gen }}(\mathbb{R})$.

Note that $K_{1, M}^{\text {gen }}=K_{0, M}^{\text {gen }}=\mathbb{G}_{m}^{M}$, and in particular $K_{1,0}^{\text {gen }}=K_{0,0}^{\text {gen }}=\operatorname{Spec}(\mathbb{Z})$.

Definition 1.16. Let $k_{Q, M}$ denote the field of fractions of $\mathcal{O}\left(K_{Q, M}\right)$. It is isomorphic to $\mathbb{Q}\left(\left(s_{i, j}\right)_{1 \leq i \leq j<Q},\left(m_{k}\right)_{1 \leq k \leq M}\right)$. In particular, $k_{0,0}=\mathbb{Q}$.

\section{Partial Factorization theorems}

The factorization theorems presented below are crucial to the construction of the cosmic Galois group. The so-called ultraviolet factorisations are used in the theory of renormalisation, the infra-red factorisations below are apparently new.

2.1. UV factorizations. Let $G$ be a connected Feynman graph, and let $\gamma \subset E_{G}$ be an edge-subgraph with connected components $\gamma_{1}, \ldots, \gamma_{n}$.

Lemma 2.1. The map $T \mapsto\left(T /(T \cap \gamma), T \cap \gamma_{1}, \ldots, T \cap \gamma_{n}\right)$ is a bijection from:

$\left\{\right.$ Spanning $k$-trees $T$ such that $\gamma_{i} \cap T$ is connected for all $\left.i=1, \ldots, n\right\}$

to

$$
\{\text { Spanning } k \text {-trees in } G / \gamma\} \times \prod_{i=1}^{n}\left\{\text { Spanning trees in } \gamma_{i}\right\}
$$


Proof. Let $T$ be any subgraph of $G$ such that $T \cap \gamma$ is a union of trees. Then

$$
h_{T /(T \cap \gamma)}=h_{T} \quad \text { and } \quad \kappa_{T /(T \cap \gamma)}=\kappa_{T} .
$$

The first formula follows from (1.15), the second is clear. If $T$ is a spanning $k$-tree such that each $T \cap \gamma_{i}$ is connected, then each $T \cap \gamma_{i}$ is a spanning tree in $\gamma_{i}$ and $T /(T \cap \gamma)$ is a spanning $k$-tree in $G / \gamma$ by $(2.1)$.

In the other direction, suppose that $S \subset G / \gamma$ is a spanning $k$-tree, and let $T_{i} \subset \gamma_{i}$ be spanning trees. There is a unique subgraph $T$ of $G$ such that $T \cap \gamma_{i}=T_{i}$ and $T /(T \cap \gamma)=S$. By $(2.1), h_{T}=h_{S}=0$ and $\kappa_{T}=\kappa_{S}=k$, and since $T$ meets every vertex of $G$ it follows that $T$ is a spanning $k$-tree.

The following factorisation formulae are essentially well-known [21].

Proposition 2.2. Let $G$ be connected, $\gamma \subset E_{G}$ as above. Then

$$
\begin{aligned}
\Psi_{G} & =\Psi_{\gamma} \Psi_{G / \gamma}+R_{\gamma, G}^{\Psi} \\
\Phi_{G}(q) & =\Psi_{\gamma} \Phi_{G / \gamma}(q)+R_{\gamma, G}^{\Phi, \mathrm{UV}}(q)
\end{aligned}
$$

where the degree of $R_{\gamma, G}^{\Psi}$ and $R_{\gamma, G}^{\Phi, \mathrm{UV}}(q)$ in the variables $\alpha_{e}, e \in E_{\gamma}$ is strictly greater than

$$
\operatorname{deg} \Psi_{\gamma}=\operatorname{deg} \prod_{i=1}^{n} \Psi_{\gamma_{i}}=h_{\gamma} .
$$

Proof. We shall prove both formulae simultaneously. Let $k=0$ (resp. 1). By (1.3) and (1.5), the set of monomials in $\Psi_{G}$ (resp. $\left.\Phi_{G}(q)\right)$ are in one-to-one correspondence with the set of spanning $k$-trees $T \subset G$. The latter can be partitioned into two subsets: those for which $T \cap \gamma_{i}$ is connected for all $i$, and those for which $T \cap \gamma_{i}$ is not connected for some $i$. The former class is in one-to-one correspondence, by lemma 2.1 , with the monomials in $\Psi_{\gamma_{1}} \ldots \Psi_{\gamma_{n}} \times \Psi_{G / \gamma}\left(\operatorname{resp} . \Psi_{\gamma_{1}} \ldots \Psi_{\gamma_{n}} \times \Phi_{G / \gamma}(q)\right)$. The latter class correspond to monomials in the remainder terms $R_{\gamma, G}^{\Psi}\left(\operatorname{resp} . R_{\gamma, G}^{\Phi, \mathrm{UV}}(q)\right)$. To see this, observe by (1.7) applied to $\gamma_{i} \cap T \subset \gamma_{i}$ that for each $i$,

$$
N_{\gamma_{i}}-N_{T \cap \gamma_{i}}=h_{\gamma_{i}}+\kappa_{T \cap \gamma_{i}}-1 \text {. }
$$

Thus the degree of the monomial $\prod_{e \notin T} \alpha_{e}$ in the variables $\alpha_{e}$ for $e \in E_{\gamma}$ is

$$
\sum_{i=1}^{n}\left(N_{\gamma_{i}}-N_{T \cap \gamma_{i}}\right)=h_{\gamma}+\sum_{i=1}^{n}\left(\kappa_{T \cap \gamma_{i}}-1\right) .
$$

This is strictly greater than $h_{\gamma}$ whenever some $T \cap \gamma_{i}$ is not connected.

Equivalently, setting $\alpha_{e}^{\prime}=\lambda \alpha_{e}$ for $e \in E_{\gamma}$ and $\alpha_{e}^{\prime}=\alpha_{e}$ otherwise, we have

$$
\begin{aligned}
\Psi_{G}\left(\alpha_{e}^{\prime}\right) & \equiv \lambda^{h_{\gamma}} \Psi_{\gamma}\left(\alpha_{e}^{\prime}\right) \Psi_{G / \gamma}\left(\alpha_{e}^{\prime}\right)\left(\bmod \lambda^{h_{\gamma}+1}\right) \\
\Phi_{G}\left(\alpha_{e}^{\prime}\right)(q) & \equiv \lambda^{h_{\gamma}} \Psi_{\gamma}\left(\alpha_{e}^{\prime}\right) \Phi_{G / \gamma}(q)\left(\alpha_{e}^{\prime}\right)\left(\bmod \lambda^{h_{\gamma}+1}\right)
\end{aligned}
$$

Remark 2.3. One can show [21] that the formulae (2.2), in the special case when $\gamma$ is a divergent subgraph, are sufficient to recover some of the main theorems of the theory of renormalization. The full strength of the factorisation formulae (2.2), for $\gamma$ an arbitrary subgraph, will only manifest itself in the motivic period. 
2.2. IR factorizations. Let $G$ be a connected Feynman graph.

With generic momenta (1.17), $\gamma \subset E_{G}$ is momentum-spanning ${ }^{8}$ if and only if

$$
\Phi_{G / \gamma}(q)=0 .
$$

This follows immediately from lemma 1.12. In this situation, the second factorization formula (2.2) is degenerate. It turns out that the remainder term $R_{\gamma, G}^{\Phi, \mathrm{UV}}$ can be further factorized via the following formula, which is apparently new.

Proposition 2.4. Let $\gamma \subset E_{G}$ be a momentum spanning edge-subgraph. Then

$$
\Phi_{G}(q)=\Phi_{\gamma}(q) \Psi_{G / \gamma}+R_{\gamma, G}^{\Phi, \mathrm{IR}}(q)
$$

where the degree of $R_{\gamma, G}^{\Phi, \mathrm{IR}}(q)$ in the variables $\alpha_{e}, e \in E_{\gamma}$ is strictly greater than

$$
\operatorname{deg} \Phi_{\gamma}(q)=h_{\gamma}+1 .
$$

Proof. Suppose that $\gamma$ has connected components $\gamma^{\prime}, \gamma_{1}, \ldots, \gamma_{n}$ such that $\gamma^{\prime}$ is momentum spanning. Monomials in $\Phi_{G}(q)$ are in one-to-one correspondence with spanning 2-trees $T=T_{1} \cup T_{2}$ such that $\left(q^{T_{1}}\right)^{2} \neq 0$. For such a 2-tree, $T \cap \gamma^{\prime}$ cannot be connected because each component $T_{i}$ intersects $\gamma^{\prime}$ non-trivially (otherwise, $V_{\gamma^{\prime}} \cap V_{T_{i}}=\emptyset$ for some $i$, which implies that $q^{T_{i}}=0$ because $\gamma^{\prime}$ is momentumspanning).

Partition the set of spanning 2-trees such that $\left(q_{1}^{T}\right)^{2} \neq 0$ into two classes: those such that $T \cap \gamma^{\prime}$ has 2 components and $T \cap \gamma_{i}$ is connected for all $i$ (call this class $C_{1}$ ), and those for which $T \cap \gamma^{\prime}$ or some $T \cap \gamma_{i}$ has strictly more components $\left(C_{2}\right)$.

There is a bijection from the first set $C_{1}$ to

$\left\{\right.$ Spanning 2-trees in $\left.\gamma^{\prime}\right\} \times\{$ Spanning trees in $G / \gamma\} \times \prod_{i}\left\{\right.$ Spanning trees in $\left.\gamma_{i}\right\}$.

It is given by the map

$$
T \mapsto\left(T \cap \gamma^{\prime},(T \cup \gamma) / \gamma, T \cap \gamma_{1}, \ldots, T \cap \gamma_{n}\right) .
$$

The proof is similar to lemma 2.1, on noting that $(T \cup \gamma) / \gamma$ is the one-vertex join of $T_{1} /\left(T_{1} \cap \gamma\right)$ and $T_{2} /\left(T_{2} \cap \gamma\right)$ along the vertex given by the image of $\gamma$, and has exactly one connected component. One checks that given a spanning 2-tree $T^{\prime} \subset \gamma^{\prime}$, and spanning trees $S \subset G / \gamma$ and $T_{i} \subset \gamma_{i}$, there is a unique graph $T \subset G$ such that $T \cap \gamma^{\prime}=T^{\prime},(T \cup \gamma) / \gamma=S$ and $T_{i}=T \cap \gamma_{i}$, and that it has exactly two connected components.

This gives a one-to-one correspondence between the set $C_{1}$ and monomials in

$$
\Phi_{\gamma}(q)=\left(\Phi_{\gamma^{\prime}}(q) \prod_{i=1}^{n} \Psi_{\gamma_{i}}\right) \Psi_{G / \gamma} .
$$

Spanning 2-trees $T$ in the set $C_{2}$ are such that $T \cap \gamma^{\prime}$ has at least 3 components, or some $T \cap \gamma_{i}$ has at least 2 components. In this case, the degree of the monomial $\prod_{e \notin T} \alpha_{e}$ in the variables $\alpha_{e}$ for $e \in E_{\gamma}$ is, by equation (1.7) applied to $T \cap \gamma^{\prime} \subset \gamma^{\prime}$ and $T \cap \gamma_{i} \subset \gamma_{i}$

$$
N_{\gamma^{\prime}}-N_{T \cap \gamma^{\prime}}+\sum_{i=1}^{n}\left(N_{\gamma_{i}}-N_{T \cap \gamma_{i}}\right)=h_{\gamma}+\left(\kappa_{T \cap \gamma^{\prime}}-1\right)+\sum_{i=1}^{n}\left(\kappa_{T \cap \gamma_{i}}-1\right),
$$

\footnotetext{
${ }^{8}$ If wants to consider non-generic momentum configurations, one could take (2.3) as the definition of momentum-spanning. But in this case the factorisation theorems stated below will fail without some additional assumptions on momenta. See example 2.5.
} 
which is strictly greater than $h_{\gamma}+1$, and contributes to $R_{\gamma, G}^{\Phi, \mathrm{IR}}(q)$.

One can derive the contraction-deletion relations (1.16) from the factorizations (2.2) and (2.4) by setting $\gamma=e$ in the former and $\gamma=G \backslash e$ in the former and latter.

Example 2.5. (Degenerate momenta). Consider the following Feynman graph

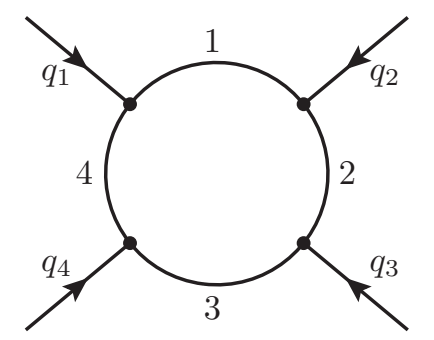

which satisfies

$\Phi_{G}(q)=\left(q_{2}+q_{3}\right)^{2} \alpha_{1} \alpha_{3}+\left(q_{1}+q_{2}\right)^{2} \alpha_{2} \alpha_{4}+q_{1}^{2} \alpha_{1} \alpha_{4}+q_{2}^{2} \alpha_{1} \alpha_{2}+q_{3}^{2} \alpha_{2} \alpha_{3}+q_{4}^{2} \alpha_{3} \alpha_{4}$

and impose the condition $\left(q_{2}+q_{3}\right)^{2}=\left(q_{1}+q_{4}\right)^{2}=0$. In this case the subgraph $\gamma$ defined by the two edges 2 and 4 satisfies $\phi_{G / \gamma}(q)=0$, but is not momentum spanning according to our stricter definition, because the incoming momenta do not all lie in the same connected component. To leading order in the subgraph variables $\alpha_{2}, \alpha_{4}$ we have

$$
\Phi_{G}(q)=\alpha_{2}\left(q_{3}^{2} \alpha_{3}+q_{2}^{2} \alpha_{1}\right)+\alpha_{4}\left(q_{1}^{2} \alpha_{1}+q_{4}^{2} \alpha_{3}\right)+R
$$

where $R=\left(q_{1}+q_{2}\right)^{2} \alpha_{2} \alpha_{4}$, and the leading terms do not factorize. If however, one further imposes the conditions $q_{2}+q_{3}=0$ and $q_{1}+q_{4}=0$ (so that the subgraph $\gamma$ now satisfies momentum conservation in each connected component), we obtain

$$
\Phi_{G}(q)=\left(q_{2}^{2} \alpha_{2}+q_{4}^{2} \alpha_{4}\right)\left(\alpha_{1}+\alpha_{3}\right)+R
$$

and a factorization formula for the leading term is restored. These types of phenomena suggest our results generalise, but will not be considered in these notes.

2.3. Factorization formulae for $\Xi$. A UV-factorization formula for $\Xi_{G}(q, m)$ follows immediately from (2.2). The IR-factorization formula requires a further constraint on the distribution of masses.

Definition 2.6. A subgraph $\gamma \subset G$ is mass-spanning if it contains all massive edges of $G$ : for every edge $e \in E_{G}$ such that $m_{e} \neq 0, e \in E_{\gamma}$.

We shall say that a subgraph $\gamma$ is mass-momentum spanning (or simply m.m. for short) if it is both mass and momentum-spanning.

For generic kinematics (1.18), a subgraph $\gamma \subset E_{G}$ satisfies

$$
\gamma \text { is } m . m . \quad \Longleftrightarrow \quad \Xi_{G / \gamma}(q, m)=0
$$

This is a direct consequence of lemma 1.13.

Theorem 2.7. Let $G$ be a connected Feynman graph, and let $\gamma \subset E_{G}$ be an edgesubgraph with any number of connected components. Then

$$
\Xi_{G}(q, m)=\Psi_{\gamma} \Xi_{G / \gamma}(q, m)+R_{\gamma, G}^{\Xi, \mathrm{UV}}(q, m)
$$


where $R_{\gamma, G}^{\Xi, \mathrm{UV}}(q, m)$ has degree $>h_{\gamma}$ in the $\alpha_{e}, e \in E_{\gamma}$. Now suppose that $\gamma$ is a mass-momentum subgraph. In this case,

$$
\Xi_{G}(q, m)=\Xi_{\gamma}(q, m) \Psi_{G / \gamma}+R_{\gamma, G}^{\Xi, \mathrm{IR}}(q, m)
$$

where $R_{\gamma, G}^{\Xi, \mathrm{IR}}(q, m)$ has degree $>h_{\gamma}+1$ in the $\alpha_{e}, e \in E_{\gamma}$.

Proof. For the proof of (2.6) combine (2.2) with the definition (1.9) and set

$$
R_{\gamma, G}^{\Xi, \mathrm{UV}}(q, m)=R_{\gamma, G}^{\Phi, \mathrm{UV}}(q)+\left(\sum_{e \in E_{\gamma}} m_{e}^{2} \alpha_{e}\right) \Psi_{\gamma} \Psi_{G / \gamma}+\left(\sum_{e \in E_{G}} m_{e}^{2} \alpha_{e}\right) R_{\gamma, G}^{\Psi}
$$

For (2.7), combine the factorization formula for $\Psi$ with the IR-factorization formula (2.4), use the condition $m_{e} \neq 0 \Rightarrow e \in E_{\gamma}$, and set

$$
R_{\gamma, G}^{\Xi, \mathrm{IR}}(q, m)=R_{\gamma, G}^{\Phi, \mathrm{IR}}(q)+\left(\sum_{e \in E_{G}} m_{e}^{2} \alpha_{e}\right) R_{\gamma, G}^{\Psi} .
$$

The degree of $R_{\gamma, G}^{\Xi, \mathrm{IR}}$ is indeed of degree $>h_{\gamma}+1$ in the variables $\alpha_{e}$, for $e \in E_{\gamma}$.

Note that the factorisation formula for $\Psi_{G}$, which is symmetric with respect to $\gamma$ and $G / \gamma$, occurs in both the UV and IR-factorizations of $\Xi_{G}$.

\section{Motic SUbGRAPHS}

For want of a better adjective, the invented word motic will be used to define a class of subgraphs of a Feynman graph. It pertains to the word mote, meaning a speck or particle (leading to notions of indivisibility), and the fact that its letters stand for 'members of the inner circle', leading to the idea of connectedness. The motive of a graph will be constructed out of its motic subquotients.

3.1. Definition of motic subgraphs. Let $G$ be a Feynman graph. Recall that at most one component of $G$ carries non-trivial kinematics.

Definition 3.1. An edge-subgraph $\Gamma \subset G$ is motic if, for every edge-subgraph $\gamma \subsetneq \Gamma$ which is mass-momentum spanning in $\Gamma$, one has $h_{\gamma}<h_{\Gamma}$.

Recall from $\S 1.1$ that any edge subgraph $\Gamma \subset G$ which is mass-momentum spanning inherits all masses and external momenta from $G$. When it is not massmomentum spanning then it is considered to be a Feynman graph with zero internal masses and no external momenta. Every subgraph of such a graph is trivially mass-momentum spanning. In particular, if $G$ has no kinematics

$$
\Gamma \subset G \text { motic } \Longleftrightarrow \Gamma \text { is } 1 \text {-particle irreducible . }
$$

Another example of a motic subgraph is a minimal mass-momentum spanning subgraph $\Gamma \subset G$ (related to the notion of 'infra-red' graph in [51]).

Remark 3.2. An edge subgraph $\Gamma \subset G$ is motic if (and only if) every massmomentum spanning edge subgraph of $\Gamma$ of the form $\Gamma \backslash e$, where $e \in E_{\Gamma}$, satisfies $h_{\Gamma \backslash e}<h_{\Gamma}$. To see that a subgraph with this property is indeed motic, let $\gamma \subset \Gamma$ be any edge subgraph which is mass-momentum spanning in $\Gamma$, and choose $e \in E_{\Gamma} \backslash E_{\gamma}$. Then $\Gamma \backslash e$ is also mass-momentum spanning in $\Gamma$ and contains $\gamma$, so we have $h_{\gamma} \leq h_{\Gamma \backslash e}<h_{\Gamma}$. Thus a graph is motic when cutting an edge either causes the loop number to drop, or breaks the property of being mass-momentum spanning. 
It follows from the definition that a subgraph $\Gamma \subset G$ is motic if and only if every connected component of $\Gamma$ is a motic subgraph of $G$.

Example 3.3. Below are the six motic subgraphs of example of 1.1. All subgraphs are mass-momentum spanning and give rise to an infra-red (and possibly also ultraviolet) sub-divergence, except for the final subgraph given by the edges 3,4 , which corresponds to a purely ultra-violet sub-divergence.
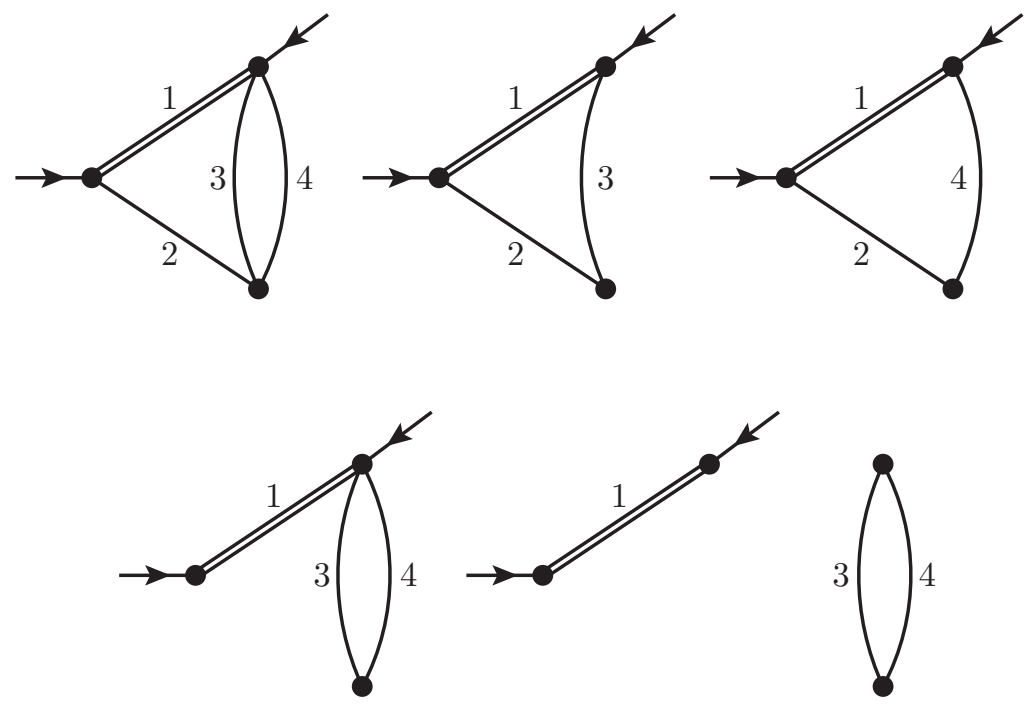

For motivation, the reader may like to check that the graph polynomial $\Xi_{G}(q, m)$ vanishes on setting $\alpha_{e}=0$ for all $e \in E_{\gamma}$, for these motic subgraphs $\gamma \subset G$.

3.2. Properties of motic subgraphs. Throughout this section, we use the abbreviation m.m. to stand for mass-momentum spanning.

Lemma 3.4. Let $\alpha \subset \beta \subset G$ be edge subgraphs.

(i). $\alpha$ is m.m. in $\beta$, and $\beta$ is m.m. in $G \Longleftrightarrow \alpha$ is m.m. in $G$.

(ii). $\beta$ is m.m. in $G \Longleftrightarrow \beta / \alpha$ is m.m. in $G / \alpha$.

Proof. Part $(i)$ is clear. For $(i i)$, observe that $(G / \alpha) /(\beta / \alpha) \cong G / \beta$ and hence $\Xi_{G / \beta}=\Xi_{(G / \alpha) /(\beta / \alpha)}$. Now apply $(2.5)$.

Note that the intersection of two $m . m$. subgraphs is not necessarily $m . m$. (in example 2.5, consider the edge subgraphs spanned by edges $1,2,3$ and 2,3,4.)

Remark 3.5. The definition of a motic subgraph is intrinsic in the following sense. If $H \subset G$ is an edge subgraph, and $\gamma \subset H$, then $\gamma$ is motic in $G$ if and only if it is motic in $H$. This follows immediately from the definition if $H$ is $m . m$. in $G$, by lemma $3.4(i)$, since $\alpha \subset \gamma$ is m.m. in $H$ if and only if it is m.m. in $G$. In the case when $H$ is not m.m. in $G$, then neither is $\gamma \subset H$ by the same lemma. It is motic if and only if it is 1-particle irreducible, which is an intrinsic property.

The main properties of motic subgraphs are summarised below.

Theorem 3.6. Motic graphs have the following properties. Let $G$ be a Feynman graph and let $\alpha, \beta \subset G$ be edge subgraphs. 
(i). (Quotients) If $\beta$ is motic in $G$, then $(\beta \cup \alpha) / \alpha$ is motic in $G / \alpha$.

(ii). (Extensions) Let $\alpha \subset \beta$. If $\alpha$ is motic in $G$ and $\beta / \alpha$ is motic in $G / \alpha$, then $\beta$ is motic in $G$.

(iii). (Unions) If $\alpha, \beta \subset G$ are motic subgraphs then $\alpha \cup \beta \subset G$ is motic.

(iv). (Contraction of edges) Let $e \in E_{G}$. If $(\alpha \cup e) / e$ is motic in $G / e$, then at least one of $\alpha$ or $\alpha \cup e$ is motic in $G$. Thus there is a surjective map

$$
\alpha \mapsto(\alpha \cup e) / e:\{\text { motic subgraphs of } G\} \longrightarrow\{\text { motic subgraphs of } G / e\} \text {. }
$$

It is not injective: it can happen that both $\alpha$ and $\alpha \cup e$ are motic.

Proof. ( $i$ ). First consider the case when $\alpha \subset \beta$, and let $\beta$ be motic in $G$. There is a one-to-one correspondence

$$
\begin{aligned}
\{\text { Edge subgraphs } \gamma \text { s.t. } \alpha \subset \gamma \subset \beta\} & \leftrightarrow\{\text { Edge subgraphs of } \beta / \alpha\} \\
\gamma & \mapsto \gamma / \alpha
\end{aligned}
$$

By lemma $3.4(i i)$, this bijection preserves the subset of m.m. subgraphs. Now by (1.15), we have $h_{\beta}=h_{\alpha}+h_{\beta / \alpha}$ and $h_{\gamma}=h_{\alpha}+h_{\gamma / \alpha}$, whence

$$
h_{\beta}-h_{\gamma}=h_{\beta / \alpha}-h_{\gamma / \alpha} .
$$

Thus $\gamma / \alpha \subset \beta / \alpha$ is strict and m.m. if and only if $\gamma \subset \beta$ is. In this case the left-hand side is strictly positive and $h_{\beta / \alpha}>h_{\gamma / \alpha}$. This proves that $\beta / \alpha$ is motic in $G / \alpha$.

Now consider the general case when $\beta \subset G$ is motic but does not necessarily contain $\alpha$. The quotient $G / \alpha$ is obtained by successively contracting edges in $e \in E_{\alpha}$. For every such edge which is an edge of $\beta$, we can invoke the case proved above. If $e$ has no common vertices with $\beta$, or a single vertex in common with $\beta$, then the image $\bar{\beta}=(\beta \cup e) / e$ of $\beta$ in $G / e$ is isomorphic to $\beta$, and the proof is straightforward. It remains to consider the case when $e$ meets $\beta$ in two vertices $v_{1}, v_{2} \in V_{\beta}$, and hence $\bar{\beta}$ is obtained from $\beta$ by identifying $v_{1}$ and $v_{2}$. We wish to show that $\bar{\beta}$ is motic. Since $E_{\beta}=E_{\bar{\beta}}$ there is a one-to-one correspondence $\gamma \leftrightarrow \bar{\gamma}$ between edge subgraphs of $\beta$ and those of $\bar{\beta}$. First of all, suppose that $v_{1}, v_{2}$ lie in two distinct connected components of $\beta$. Euler's formula (1.2) implies that $h_{\bar{\beta}}=h_{\beta}$. Since $\beta$ is motic, all momentum-bearing vertices lie in a single connected component of $\beta$. Thus a strict subgraph $\bar{\gamma} \subsetneq \bar{\beta}$ is m.m. if and only if $\gamma \subsetneq \beta$ is m.m. and we have

$$
h_{\bar{\gamma}}=h_{\gamma}<h_{\beta}=h_{\bar{\beta}}
$$

using the fact that $\beta$ is motic. This proves that $\bar{\beta}$ is motic also. Now suppose that $v_{1}, v_{2}$ lie in the same connected component of $\beta$, and hence $h_{\bar{\beta}}=h_{\beta}+1$ by (1.2). Let $\bar{\gamma} \subsetneq \bar{\beta}$ be an $m$.m. edge-subgraph. If $\gamma$ is $m . m$. in $\beta$, we deduce that

$$
h_{\bar{\gamma}} \stackrel{(1.2)}{\leq} h_{\gamma}+1<h_{\beta}+1=h_{\bar{\beta}},
$$

since the middle inequality follows from the fact that $\beta$ is motic. Now suppose that $\gamma$ is not $m . m$. in $\beta$. Since $\gamma$ is mass-spanning, the set of momentum bearing vertices of $\beta$ do not all lie in the same connected component of $\gamma$. Since $\bar{\gamma}$ is m.m., their images in $\bar{\gamma}$ lie in the same connected component, and we have $\kappa_{\gamma}>\kappa_{\bar{\gamma}}$ and hence $h_{\bar{\gamma}}=h_{\gamma}$ by (1.2). But then $h_{\bar{\gamma}}=h_{\gamma} \leq h_{\beta}<h_{\bar{\beta}}$ since $h_{\bar{\beta}}=h_{\beta}+1$. In both cases we have shown that $h_{\bar{\gamma}}<h_{\bar{\beta}}$, which proves that $\bar{\beta}$ is motic. 
(ii). Let $\alpha \subset \beta$ be motic and $\beta / \alpha \subset G / \alpha$ be motic also. Let $\gamma \subsetneq \beta$ be a strict m.m. edge subgraph. Denote its image in $\beta / \alpha$ by

$$
\bar{\gamma}=(\gamma \cup \alpha) / \alpha
$$

By lemma $3.4(i), \gamma$ and $\gamma \cup \alpha$ are m.m. in $\beta$ and hence by lemma $3.4(i i), \bar{\gamma}$ is $m . m$. in $\beta / \alpha$. By (1.15), we have

$$
h_{\gamma}=h_{\gamma \cap \alpha}+h_{\gamma /(\gamma \cap \alpha)} \leq h_{\gamma \cap \alpha}+h_{\bar{\gamma}} \leq h_{\alpha}+h_{\beta / \alpha}=h_{\beta}
$$

The first inequality holds because $\bar{\gamma}$ is obtained from $\gamma /(\gamma \cap \alpha)$ by identifying vertices and therefore $h_{\gamma /(\gamma \cap \alpha)} \leq h_{\bar{\gamma}}$. Suppose by contradiction that $h_{\gamma}=h_{\beta}$. Then

$$
h_{\bar{\gamma}}=h_{\beta / \alpha} \quad, \quad h_{\gamma \cap \alpha}=h_{\alpha} \quad, \quad h_{\gamma /(\gamma \cap \alpha)}=h_{\bar{\gamma}} .
$$

Since $\beta / \alpha$ is motic and $\bar{\gamma} \subset \beta / \alpha$ is m.m., the first equality implies that $\bar{\gamma}=\beta / \alpha$. Suppose that $\alpha \cap \gamma$ is m.m. in $\alpha$. Then the second equality would imply that $\alpha \cap \gamma=\alpha$, since $\alpha$ is motic, and this, together with $\bar{\gamma}=\beta / \alpha$, contradicts the fact that $\gamma$ is a strict subgraph of $\beta$.

Now consider the case when $\alpha \cap \gamma$ is m.m. in $\alpha$. If $\alpha$ is not m.m. in $G$, then every subgraph of $\alpha$ is automatically m.m. in $\alpha$ and there is nothing to prove. Therefore suppose that $\alpha$ is m.m. in $G$, and hence in $\beta$. Consider the set $Q$ of momentum-bearing vertices in $\beta$, and suppose that they lie in $k \geq 1$ different connected components of $\alpha \cap \gamma$. It suffices to show that $k=1$, since in that case $\alpha \cap \gamma$ is m.m. from the definition (as both $\gamma$ and $\alpha$ are m.m. in $\beta$ ). Since $\gamma$ is momentum-spanning, the image of $Q$ in $\gamma /(\gamma \cap \alpha)$ consists of exactly $k$ vertices lying in a single connected component. On the other hand, since $\alpha$ is momentumspanning, the image of $Q$ in $\bar{\gamma}$ reduces to a single vertex. So $\bar{\gamma}$ is obtained from $\gamma /(\gamma \cap \alpha)$ by identifying these $k$ connected vertices, and possibly identifying further vertices. If $k>1$ we would have $h_{\gamma /(\gamma \cap \alpha)}<h_{\bar{\gamma}}$ by (1.2) contradicting the third equality above. Hence $k=1$, as required.

(iii). By $(i)$, the graph $\bar{\beta}=(\alpha \cup \beta) / \alpha$ is motic in $G / \alpha$. Since $\alpha \subset \alpha \cup \beta$, it follows from the extension property (ii) that $\alpha \cup \beta$ is motic in $G$.

(iv). Let $\alpha \subset G$ be an edge subgraph, and $e \in E_{G}$. Let $\Gamma=\alpha \cup e$ and suppose that $\Gamma / e$ is motic in $G / e$. Suppose that $e$ is not a tadpole. By the proof of $(i)$ above, there is a one-to-one correspondence between m.m. subgraphs $\gamma \subset \Gamma$ which contain the edge $e$ and m.m. subgraphs $\gamma / e$ of $\Gamma / e$. If $\gamma \subsetneq \Gamma$ is strict and contains the edge $e$, then $h_{\gamma}=h_{\gamma / e}<h_{\Gamma / e}=h_{\Gamma}$ by (1.15). The strict inequality in the middle follows since $\Gamma / e$ is motic. By remark 3.2, $\Gamma$ will be motic if $\Gamma \backslash e$ is not m.m., or if it is m.m. and $h_{\Gamma \backslash e}<h_{\Gamma}$.

Therefore let us suppose that $\Gamma \backslash e$ is m.m. and $h_{\Gamma \backslash e}=h_{\Gamma}$. The latter equality implies that $e$ is a bridge in $\Gamma$, so we can write $\Gamma \backslash e=\Gamma_{1} \cup \Gamma_{2}$, where $\Gamma_{1}, \Gamma_{2}$ are disjoint. The quotient $\Gamma / e$ is the one-vertex join $\Gamma_{1} \cdot \Gamma_{2}$. Now there is a bijection between the subgraphs $\gamma_{1} \cup \gamma_{2}$ of $\Gamma_{1} \cup \Gamma_{2}$ and $\gamma_{1} \cdot \gamma_{2}$ of $\Gamma_{1} \cdot \Gamma_{2}$. This bijection preserves the number of loops and the property of being m.m.. The momentum spanning property follows from the fact that $\Gamma \backslash e$ is momentum-spanning by assumption, so all momenta flow into only one of the parts $\Gamma_{i}$. Thus if $\Gamma_{1} \cdot \Gamma_{2}$ is motic if and only if $\Gamma_{1} \cup \Gamma_{2}$ is. Since $\Gamma / e$ is motic we deduce that $\Gamma \backslash e$ is. It remains to consider the case when $e$ is a tadpole. Then $\Gamma \backslash e=\Gamma / e$ is motic (so too is $\Gamma$ ). 


\section{The motic Hopf Algebra of GRAphs}

The notion of motic subgraph gives rise to a Hopf algebra which encapsulates both infra-red and ultra-violet divergences of Feynman graphs.

Definition 4.1. Let $\mathcal{F}$ denote the free $\mathbb{Z}$-module generated by disjoint unions of motic $^{9}$ Feynman graphs, modulo the equivalence relation $G \sim G \cup\{v\}$ where $v$ is an isolated vertex. It is bigraded by

$$
\text { (number of edges, number of loops). }
$$

The disjoint union of graphs defines a commutative ring structure on $\mathcal{F}$, whose unit is the empty graph 1 . Define a coproduct on $\mathcal{F}$ by the formula:

$$
\begin{aligned}
\Delta: \mathcal{F} & \longrightarrow \mathcal{F} \otimes_{\mathbb{Z}} \mathcal{F} \\
G & \mapsto \sum_{\gamma \subset E_{G}} \gamma \otimes G / \gamma
\end{aligned}
$$

where $G$ is a connected graph and the sum is over all motic subgraphs $\gamma$ of $G$. This is a generalisation of the Connes-Kreimer coproduct for scalar graphs. The map $\Delta$ extends to a unique homomorphism on $\mathcal{F}$.

Theorem 4.2. $\mathcal{F}$ is a connected bigraded Hopf algebra.

Proof. The coassociativity of $\Delta$ is a consequence of properties $(i)$ and $(i i)$ of theorem 3.6 by a standard argument (see, for example, [11]). The augmentation map $\varepsilon$ is the projection $\mathcal{F} \rightarrow \mathcal{F}_{0} \cong \mathbb{Z}$. Denote its kernel by $I$. A generator $G$ is motic, so $\Delta(G)=1 \otimes G+G \otimes 1(\bmod I \otimes \mathcal{F}+\mathcal{F} \otimes I)$. From this follows the equations $(\varepsilon \otimes \mathrm{id}) \Delta=\mathrm{id}$ and $(\mathrm{id} \otimes \varepsilon) \Delta=\mathrm{id}$. The antipode $S$ is constructed by the usual recursive formula for the antipode in a connected graded commutative Hopf algebra, and is defined over $\mathbb{Z}$. The fact that the loop number and edge number are gradings follows from (1.15) and $N_{\gamma}+N_{G / \gamma}=N_{G}$.

The grading by loop number is of importance when considering the geometry of graphs in momentum space and the perturbative expansion, but the edge number grading will be more relevant in these notes, since we shall focus on the geometry of graphs in parametric space and their corresponding motives.

Let us denote by $\mathcal{F}_{Q, M}$ the free submodule of $\mathcal{F}$ generated by motic Feynman graphs of type $(Q, M)$, i.e., with $Q$ external momenta and $M$ possible non-zero masses. Then $\mathcal{F}_{Q, M}$ is a module over $\mathcal{F}_{0,0}$, where multiplication is that of $\mathcal{F}$ :

$$
\mathcal{F}_{0,0} \otimes_{\mathbb{Z}} \mathcal{F}_{Q, M} \longrightarrow \mathcal{F}_{Q, M}
$$

In particular, $\mathcal{F}_{0,0}$ is a commutative ring. An important feature of the motic coproduct is the 'all or nothing' property of masses and momenta:

$$
\Delta \mathcal{F}_{Q, M} \subset\left(\mathcal{F}_{Q, M} \otimes_{\mathbb{Z}} \mathcal{F}_{0,0}\right) \oplus\left(\mathcal{F}_{0,0} \otimes_{\mathbb{Z}} \mathcal{F}_{Q, M}\right)
$$

The terms landing in the first factor correspond to subgraphs which are m.m., and the terms in the second factor are those which are not m.m.. In particular, $\mathcal{F}_{0,0} \subset \mathcal{F}$ is a Hopf subalgebra. It was defined in [11] and called the core Hopf algebra.

There is a variant of this construction in which one considers graphs whose edges have distinct labels. We leave the details to the reader.

\footnotetext{
${ }^{9} G$ is motic if it is motic as a subgraph of itself.
} 
Remark 4.3. A more fundamental structure underlying the space of graphs should be the structure of an operad. For graphs of type $(0,0)$ it is clear how to define this by insertion into vertices of graphs, but is more delicate for graphs with masses and momenta. See [38] for some related categorical notions.

4.1. Coradical filtration. The coradical filtration $C_{i} \mathcal{F}$ is defined as follows. Let

$$
\Delta^{\prime}=\Delta-1 \otimes \mathrm{id}-\mathrm{id} \otimes 1
$$

denote the reduced coproduct. Then $C_{0} \mathcal{F}=\mathbb{Z}$ and $x \in C_{n} \mathcal{F}$, for $n \geq 1$, if and only if $\left(\Delta^{\prime}\right)^{n} x=0$. The space $C_{1} \mathcal{F}$ consists of primitive linear combinations of (unions of) graphs.

Lemma 4.4. The coradical filtration of a motic Feynman graph $G$ is $\leq h_{G}$ if $G$ is of type $(0,0)$ and $\leq h_{G}+1$ otherwise.

Proof. A motic graph of type $(0,0)$ is $1 \mathrm{PI}$ and hence satisfies $h_{\gamma} \geq 1$ (for every edge $e$ of $\gamma$ we have $\left.0 \leq h_{\gamma \backslash e}<h_{\gamma}\right)$. Let $G$ be a generator of $\mathcal{F}_{0,0}$. Then $\left(\Delta^{\prime}\right)^{n} G \subset \mathcal{F}_{0,0}^{\otimes n+1}$ and each component is motic so has loop number $\geq 1$. Since the loop number is a grading, this forces $\left(\Delta^{\prime}\right)^{n}$ to vanish if $n \geq h_{G}$.

In the general case, let $G$ be of type $(Q, M)$. Then by $(4.3)$

$$
\left(\Delta^{\prime} G\right)^{n} \subset \mathcal{F}_{0,0}^{\otimes n} \otimes_{\mathbb{Z}} \mathcal{F}_{P, Q}
$$

and the same argument shows that $\left(\Delta^{\prime} G\right)^{n}$ vanishes if $n \geq h_{G}+1$, the only difference being that the component in $\mathcal{F}_{P, Q}$ can satisfy $h_{\gamma}=0$.

4.2. Motic descendents of graphs. If $G$ is a motic graph, define

$$
\begin{aligned}
& d_{e}(G)=G / e \quad \text { if } e \in E_{G} \\
& d_{\gamma}(G)=\gamma \otimes G / \gamma \quad \text { if } \gamma \subsetneq E_{G} \text { is motic. }
\end{aligned}
$$

Iterating these operations generates a cascade of tensor products of motic graphs.

Definition 4.5. Let $T(\mathcal{F})=\bigoplus_{m \geq 0} \mathcal{F}^{\otimes m}$ denote the tensor algebra on $\mathcal{F}$. The grading by edge numbers induces a grading we shall call the degree:

$$
\operatorname{deg}\left(\gamma_{1} \otimes \ldots \otimes \gamma_{n}\right)=\sum_{i=1}^{n}\left(N_{\gamma_{i}}-1\right)=N_{\cup \gamma_{i}}-n .
$$

Given a motic graph $G$, define the set of motic descendants $D(G)$ of $G$ to be the set of tensor products of graphs (generators in $T(\mathcal{F})$ ) obtained by repeatedly applying operators of the form id ${ }^{\otimes r} \otimes d_{\bullet} \otimes \mathrm{id}^{\otimes s}$ to $G$, where $d_{\bullet}$ is one of the two operations (4.4) above. Since these strictly decrease the degree, the set $D(G)$ is finite.

The degree (4.5) is the usual grading in the literature on the bar construction. It will correspond to the dimension of facets in the Feynman polytope and also to the cohomological degree of our graph motives (to be defined below).

Remark 4.6. The operator $d_{e}$ is not to be confused with the contraction of $e$, defined by $c_{e} G=G / / e$. Then the equation

$$
\Delta c_{e}=\left(c_{e} \otimes \mathrm{id}+\mathrm{id} \otimes c_{e}\right) \Delta
$$

is not always satisfied, so $\mathcal{F}$ is not a differential Hopf algebra in general. The reason is the failure of the map in theorem $3.6(i v)$ to be injective. More precisely, if $G$ is a Feynman graph, and $\Gamma$ an edge subgraph such that $\Gamma$ and $\Gamma \backslash e$ are both motic in $G$ with $h_{\Gamma}=h_{\Gamma \backslash e}$, then $c_{e}$ is not necessarily zero on $(\Gamma \backslash e) / \Gamma$, and (4.6) fails. This 
can only occur if $\Gamma$ is $m . m$. and $\Gamma \backslash e$ is not (for example, let $\Gamma$ be the subgraph spanned by edges $1,3,4$ in example 1.1 , and $e=1)$. For $G$ of type $(0,0)$ equation (4.6) is satisfied and $\mathcal{F}_{0,0}$ can be made into a differential graded Hopf algebra [11].

4.3. Uniqueness of graph polynomials. This section is not essential and can be skipped. It shows that the graph polynomials $\Psi_{G}$ and $\Xi_{G}$ are nearly uniquely determined by the factorization and edge-contraction formulae.

Lemma 4.7. Let $\Gamma \subset G$ be an edge-subgraph. Then there exists a $1 P I$ and hence motic subgraph $\gamma \subseteq \Gamma$ such that $h_{\gamma}=h_{\Gamma}$.

Proof. Let $e$ be an edge of $\Gamma$. If $h_{\Gamma \backslash e}=h_{\Gamma}$ then replace $\Gamma$ with $\Gamma \backslash e$. Repeat until we obtain a graph $\gamma$ whose loop number drops whenever any edge is cut.

Proposition 4.8. For every labelled motic Feynman graph $G$, let

$$
P_{G}, C_{G} \in \mathbb{Q}\left[\alpha_{e}, e \in E_{G}\right]
$$

be homogeneous polynomials of degrees $h_{G}, h_{G}+1$ respectively, which respect the equivalence relation of $\S 1.1$ and take the same values on $G$ and $G \cup\{v\}$ where $v$ is an isolated vertex, and satisfy the following properties:

(1) Partial factorisations:

$$
\begin{array}{llll}
P_{G} & \equiv_{\gamma} & P_{\gamma} P_{G / \gamma} & \\
C_{G} & \equiv_{\gamma} & P_{\gamma} C_{G / \gamma} & \text { if } \gamma \text { not m.m. } \\
C_{G} & \equiv_{\gamma} & C_{\gamma} P_{G / \gamma} & \text { if } \gamma \text { is m.m. }
\end{array}
$$

where $A_{G} \equiv_{\gamma} B_{\gamma} C_{G / \gamma}$ for homogeneous polynomials $A, B, C$ means that $A_{G}-B_{\gamma} C_{G / \gamma}$ is of degree $>\operatorname{deg} B_{\gamma}$ in the variables $\alpha_{e}$, for $e \in E_{\gamma}$.

(2) Edge contraction:

$$
\begin{aligned}
\left.P_{G}\right|_{\alpha_{e}=0} & =P_{G / / e} \\
\left.C_{G}\right|_{\alpha_{e}=0} & =C_{G / / e}
\end{aligned}
$$

(3) Initial conditions: if $G$ has a single edge then $P_{G}=\Psi_{G}$ and $C_{G}=\Xi_{G}(q, m)$. If $G$ is a banana graph (a connected graph with 2 vertices) with all edges massive, then the coefficient of $\prod_{e \in E_{G}} \alpha_{e}$ in $C_{G}$ is

$$
q^{2}+\sum_{e \in E_{G}} m_{e}^{2} .
$$

With these assumptions, $P_{G}=\Psi_{G}$ and $C_{G}=\Xi_{G}(q, m)$.

Proof. The proof proceeds by induction on the number of edges. Assume for now that the theorem is true for all motic graphs $G$ such that $h_{G} \leq 1$. For the induction step, suppose that $G$ is motic and satisfies $h_{G} \geq 2$. For any edge $e_{0} \in E_{G}$, there exists a non-trivial motic subgraph $\gamma \subset G$ such that $e_{0} \notin E_{\gamma}$ and $h_{\gamma}=h_{G}-1$, by lemma 4.7. It may or may not be m.m.. We obtain by induction hypothesis

$$
P_{G}=\Psi_{\gamma} \Psi_{G / \gamma}+R_{\gamma, G / \gamma}^{P}
$$

on application of (1), where the degree of $R_{\gamma, G / \gamma}^{P}$ in the variables $\alpha_{e}$, for $e \in \gamma$ is $\geq h_{\gamma}+1$ and hence equal to $h_{G}$. Thus $R_{\gamma, G / \gamma}^{P}$ does not depend on the variables $\alpha_{e}$, for $e \in G / \gamma$. We deduce that $P_{G}$ is of degree $\leq 1$ in $\alpha_{e_{0}}$ and the coefficient of $\alpha_{e_{0}}$ is $\Psi_{\gamma} \Psi_{G / \gamma}^{e_{0}}$. The constant term in $\alpha_{e_{0}}$ is uniquely determined from (2) and induction 
hypothesis since $G / / e_{0}$ has fewer edges. This proves that $P_{G}=\Psi_{G}$ for all $G$. For the polynomial $C_{G}$, an application of (1) and the induction hypothesis gives either

$$
\begin{aligned}
C_{G} & =\Xi_{\gamma}(q, m) \Psi_{G / \gamma}+R_{\gamma, G / \gamma}^{C, \mathrm{IR}} \\
\text { or } \quad C_{G} & =\Psi_{\gamma} \Xi_{G / \gamma}(q, m)+R_{\gamma, G / \gamma}^{C, \mathrm{UV}}
\end{aligned}
$$

depending on whether $\gamma$ is $m$.m. or not. The former case proceeds as for $P_{G}$. In the latter case, the term $R_{\gamma, G / \gamma}^{C, \mathrm{UV}}$ is of degree at most one in $\alpha_{e_{0}}$, and hence the coefficients of $\left(\alpha_{e_{0}}\right)^{k}$ for $k \geq 2$ are uniquely determined by induction. The coefficients of $\left(\alpha_{e_{0}}\right)^{0}$ are determined by contracting the edge $e_{0}$ via (2). Thus the only undetermined term in $C_{G}$ is the unique monomial $\prod_{e} \alpha_{e}$ of degree exactly one in every $\alpha_{e}$ for $e \in E_{G}$. It can only occur in $C_{G}$ if $h_{G}+1=\operatorname{deg} C_{G}=N_{G}$. If $G$ has more than one component, it necessarily contains a self-edge, say $e^{\prime}$, which is a motic subgraph and not m.m.. Applying (1) to this subgraph gives

$$
C_{G}=\alpha_{e^{\prime}} C_{G / e^{\prime}}(q, m)+O\left(\alpha_{e^{\prime}}^{2}\right)
$$

and so the term linear in $\alpha_{e^{\prime}}$ is again determined by induction. The only remaining case is when $G$ is connected. Then $N_{G}=h_{G}+1$ implies that it has two vertices by Euler's formula (1.2), so it is a banana graph. Furthermore, every edge is massive otherwise we could construct a non-trivial motic m.m. subgraph $\gamma$, and determine $C_{G}$ using the third formula of (1). Thus we are reduced to the case of (3).

It remains to check the cases when $G$ does not have a non-trivial motic subgraph, i.e., $h_{G} \leq 1$. First suppose that $G$ is a forest $\left(h_{G}=0\right.$ ). Then $P_{G}$ is constant (of degree 0 ), and by contracting edges we deduce that $P_{G}=P_{\{v\}}=1$, where $v$ is an isolated vertex. The polynomial $C_{G}$ is homogeneous of degree one, i.e., $C_{G}=\sum_{e} \lambda_{e} \alpha_{e}$. The coefficient $\lambda_{e}$ is uniquely determined by contracting all edges except $e$, and we are reduced to the case of a graph with a single edge (3). Now suppose that $h_{G}=1$. The same argument proves the statement for $P_{G}$. The polynomial $C_{G}$ is homogeneous of degree two and hence of the form $C_{G}=\sum_{e, f} \lambda_{e, f} \alpha_{e} \alpha_{f}$. By contracting all edges except $e, f$ we reduce to a two-edge graph. By edge contraction, $\lambda_{e, e}$ and $\lambda_{f, f}$ are determined via (3). If $e, f$ form a massive 2 -edge banana, the coefficient $\lambda_{e, f}$ is determined by the second part of (3). In all other cases, $G$ has a non-trivial motic m.m. subgraph and we can reduce to a graph with fewer edges by the partial factorization formulae as above.

Remark 4.9. Properties (1) and (2) are essential requirements for the productstructure on graph hypersurfaces and hence for our results on the action of the cosmic Galois group. Thinking of the data of $P$ and $C$ as Feynman rules, the proposition tells us how restrictive these requirements are. The polynomial $P_{G}$ is essentially uniquely determined, but there is nonetheless a small amount of freedom to modify the polynomial $C_{G}$ by adding a term

$$
s_{n} \prod_{e \in E_{G}} \alpha_{e}
$$

to every massive banana graph $G$ with $n$ loops, where $s_{n}$ is a new parameter. By properties (1) and (2), the coefficients $s_{n}$ will infiltrate the $C_{G}$ for all other Feynman graphs. In this way one could, suprisingly, modify the Feynman integrands by essentially a single quantity $s_{n}$ (which could depend on the labelling of the edges $\left.E_{G}\right)$ at every loop order without affecting the mathematical structures studied in 
this paper. ${ }^{10}$ Observe also that the concept of mass and momenta only enter via the initial conditions (3). One could allow for more diverse families of polynomials $P_{G}, C_{G}$ either by restricting the set of graphs under consideration, or by allowing the polynomials $P_{G}, C_{G}$ to have higher degrees.

An interesting question would be to study similar partial factorisation properties of Feynman integrands for gauge theories and see what restrictions this imposes on the set of possible Feynman rules.

\section{LinEAR BLOW-UPS IN PROJECTIVE SPACE}

We study blow-ups of coordinate linear subspaces of projective space. The role of local coordinates is emphasised owing to their close relation to sector decompositions in the physics literature.

5.1. Iterated blow-ups. Let $S$ be a finite set, and let $\mathbb{P}^{S}=\mathbb{P}\left(\mathbb{Q}^{S}\right)$ denote projective space over $\mathbb{Q}$ of dimension $|S|-1$ with projective coordinates $\alpha_{s}, s \in S$. Every subset $I \subset S$ defines a linear subspace

$$
L_{I} \cong \mathbb{P}^{I^{c}} \subset \mathbb{P}^{S}
$$

defined by the vanishing of coordinates $\alpha_{i}, i \in I$. The notation $I^{c}$ denotes the complement $S \backslash I$ when the set $S$ is unambiguous. We have

$$
L_{I_{1}} \cap L_{I_{2}}=L_{I_{1} \cup I_{2}} .
$$

Now let $B \subset 2^{S}$ be a set of subsets of $S$ with the property that

$$
I_{1}, I_{2} \in B \quad \Longrightarrow \quad I_{1} \cup I_{2} \in B
$$

and satisfying $S \in B$. Define the iterated blow-up $P^{B}$ of $\mathbb{P}^{S}$ along $B$

$$
\pi_{B}: P^{B} \longrightarrow \mathbb{P}^{S}
$$

by the following standard procedure:

(0) First blow up all subspaces $L_{I}$, for $I \in B$, such that $\operatorname{dim}\left(L_{I}\right)=0$, in any order, to obtain a space $P_{0} \rightarrow \mathbb{P}^{S}$.

(1) Blow up all strict transforms of $L_{I}$ in $P_{0}$, for $I \in B$, such that $\operatorname{dim}\left(L_{I}\right)=1$, in any order, to obtain a space $P_{1} \rightarrow P_{0}$.

(k) At the $k^{\text {th }}$ stage, blow up the strict transforms of $L_{I}$ in $P_{k-1}$, for $I \in B$ such that $\operatorname{dim}\left(L_{I}\right)=k$, to obtain $P_{k} \rightarrow P_{k-1}$.

Finally, define $P^{B}=P_{|S|-1}$. The key point is that since $B$ is closed under unions (5.1), the strict transforms of $L_{I}$ in $P_{k-1}$ for $I \in B$ are disjoint and can be blown up in any order, and thus $P^{B}$ is well-defined. The scheme $P^{B}$ has a divisor

$$
D=\pi^{-1}\left(\bigcup_{i \in S} L_{i}\right)
$$

given by the total transform of the coordinate hyperplanes $L_{i}$. If $U_{I} \subset L_{I}$ denotes the open where $\alpha_{j} \neq 0$ for all $j \notin I$, the irreducible components of $D$ are

$$
\begin{aligned}
& D_{i}=\overline{\pi^{-1}\left(U_{i}\right)} \quad, \text { for all } i \in S \text {. } \\
& D_{I}=\overline{\pi^{-1}\left(U_{I}\right)} \quad, \text { for all } I \in B, \text { where } 2 \leq|I| \leq|S|-1
\end{aligned}
$$

\footnotetext{
${ }^{10}$ This provides Feynman integrals with a new and natural parametrisation, distinct from the kinematic parameters, which may be useful for setting up differential equations.
} 
where the closure is with respect to the Zariski topology. By taking repeated intersections, $D$ defines a stratification on $P^{B}$. For every $I \in B$ define

$$
\begin{aligned}
& B^{I}=\{J \in B \text { such that } J \subseteq I\} \\
& B_{I}=\{J \backslash I \text { where } J \in B \text { and } J \supseteq I\},
\end{aligned}
$$

and for every $i \in S$, set

$$
B_{i}=\{J \backslash(J \cap\{i\}) \text { for } J \in B\} .
$$

Theorem 5.1. The space $P^{B}$ is a smooth scheme over $\mathbb{Z}$ and is well-defined (it does not depend on the order of blow-ups at each stage of the above procedure).

The divisor $D$ is strict normal crossing, and there are canonical isomorphisms

$$
\begin{aligned}
D_{I} & =P^{B^{I}} \times P^{B_{I}} \quad \text { where } I \in B \\
D_{i} & =\text { Spec } \mathbb{Z} \times P^{B_{i}} .
\end{aligned}
$$

Consider any two components $D_{I}, D_{J}$ (of either type (5.3)), where $I, J \in B$ or a singleton in $S$. Then $D_{I} \cap D_{J}$ is non-empty if and only if either

$$
I \subset J \quad \text { or } \quad J \subset I
$$

or

$$
I \cap J=\emptyset \quad \text { and } \quad I \cup J \notin B .
$$

The latter case only arises when at least one of $I, J$ is a singleton in $S$, by (5.1).

A proof is outlined below.

5.2. B-polytope. Define a compact real manifold with corners

$$
\widetilde{\sigma}_{B} \subset P^{B}(\mathbb{R})
$$

to be the closure, in the analytic topology, of $\pi_{B}^{-1}(\stackrel{\circ}{\sigma})$, where $\stackrel{\circ}{\sigma} \subset \mathbb{P}^{S}(\mathbb{R})$ is the open coordinate simplex defined by $\alpha_{i}>0$, for all $i \in S$. Its facets inherit the following product structure from the isomorphisms (5.5) of theorem 5.1.

Corollary 5.2. The facets of $\widetilde{\sigma}_{B}$ satisfy

$$
\begin{array}{rlrl}
\widetilde{\sigma}_{B} \cap D_{i}(\mathbb{C}) & =\{p t\} \times \widetilde{\sigma}_{B_{i}} & & \text { for } i \in S \\
\widetilde{\sigma}_{B} \cap D_{I}(\mathbb{C}) & =\widetilde{\sigma}_{B^{I}} \times \widetilde{\sigma}_{B_{I}} & \text { for } I \in B, 2 \leq|I| \leq|S|-1 .
\end{array}
$$

The poset structure on the faces of $\widetilde{\sigma}_{B}$, with respect to inclusion, is identical to the poset structure on the stratification of $P^{B}$ generated by the divisor $D$.

5.3. Local coordinates and theorem 5.1. The space $P^{B}$ will be covered by explicit coordinate charts of the form

$$
\mathbb{A}^{n}=\operatorname{Spec} \mathbb{Z}\left[\beta_{1}, \ldots, \beta_{n}\right] .
$$

These charts are obtained by iterating the following basic example.

Example 5.3. Consider a single blow-up in affine space. If $J \subset\{1, \ldots, n\}$ let $L_{J}$ denote the zero locus of $\alpha_{j}, j \in J$. Let $I=\{1, \ldots, m\} \subset\{1, \ldots, n\}$. The blow-up $A \rightarrow \mathbb{A}^{n}$ of $\mathbb{A}^{n}$ along $L_{I}$ has local coordinates

$\beta_{1}=\frac{\alpha_{1}}{\alpha_{m}}, \beta_{2}=\frac{\alpha_{2}}{\alpha_{m}}, \ldots, \beta_{m-1}=\frac{\alpha_{m-1}}{\alpha_{m}}, \beta_{m}=\alpha_{m}, \beta_{m+1}=\alpha_{m+1}, \ldots, \beta_{n}=\alpha_{n}$.

This means that there is an affine chart $\mathbb{A}^{n} \subset A$ with a morphism

$$
\pi: \operatorname{Spec} \mathbb{Z}\left[\beta_{1}, \ldots, \beta_{n}\right] \longrightarrow \operatorname{Spec} \mathbb{Z}\left[\alpha_{1}, \ldots, \alpha_{n}\right]
$$


defined by $\pi^{*}\left(\alpha_{i}\right)=\beta_{i} \beta_{m}$ for $1 \leq i<m$ and $\pi^{*}\left(\alpha_{i}\right)=\beta_{i}$ for $i \geq m$. It is an isomorphism on the opens defined by $\beta_{m} \neq 0$ and $\alpha_{m} \neq 0$ respectively. The exceptional divisor in these coordinates is given by the equation $\beta_{m}=0$, and the strict transform of $L_{i}$ is given by $\beta_{i}=0$ for all $i \neq m$. The strict transform of $L_{m}$ in $A$ does not meet this coordinate chart, and hence neither does the strict transform of any $L_{J}$, for $m \in J \subsetneq I$.

More generally, for any choice of element $j \in I$, we have local coordinates

$$
\beta_{i}=\frac{\alpha_{i}}{\alpha_{j}} \text { for } i \in I \backslash\{j\}, \quad \beta_{j}=\alpha_{j}, \quad \beta_{i}=\alpha_{i} \text { for } i \notin I
$$

and hence a local chart Spec $\mathbb{Z}\left[\beta_{1}, \ldots, \beta_{n}\right]$ on $A$ as above. These form an affine covering of $A$. Note that if $J_{1}, J_{2} \subset I$ and $J_{1} \cup J_{2}=I$, then the strict transforms of $L_{J_{1}}$ and $L_{J_{2}}$ do not intersect in $A$, since this is true in every coordinate chart.

We now define a scheme, denoted $P^{B}$, explicitly using such affine charts. It will turn out to be isomorphic to the space defined in the previous section.

For every nested sequence (or flag)

$$
\mathcal{F}: \quad \emptyset=I_{0} \subsetneq I_{1} \subsetneq I_{2} \subsetneq \ldots \subsetneq I_{k} \subsetneq I_{k+1}=S
$$

where each $I_{r} \in B$, and every choice of elements

$$
c: \quad j_{n} \in I_{n} \backslash I_{n-1} \quad \text { for } \quad 1 \leq n \leq k+1,
$$

which are maximal in the sense that $\mathcal{F}, c$ cannot be made larger (i.e., there exists no $I \in B$ and $0 \leq i \leq k$ such that $I_{i} \subsetneq I \subsetneq I_{i+1}$ and $\left.j_{i+1} \notin I\right)$, define an affine

$$
\mathbb{A}^{\mathcal{F}, c}=\operatorname{Spec} \mathbb{Z}\left[\beta_{i}^{\mathcal{F}, c}, i \neq j_{k+1}\right]
$$

and a morphism $\pi: \mathbb{A}^{\mathcal{F}, c} \rightarrow \operatorname{Spec} \mathbb{Z}\left[\alpha_{i}, i \neq j_{k+1}\right]$ where the right-hand side is the open subset $\alpha_{j_{k+1}}=1$ in $\mathbb{P}^{S}$. The morphism $\pi$ is defined as follows. Its inverse $\left(\pi^{-1}\right)^{*}$ on the open $\alpha_{j_{n}} \neq 0$ for all $1 \leq n \leq k$ is given by

$$
\begin{aligned}
& \beta_{i}^{\mathcal{F}, c}=\frac{\alpha_{i}}{\alpha_{j_{n}}} \quad \text { for } \quad i \in I_{n} \backslash\left(\left\{j_{n}\right\} \cup I_{n-1}\right), \quad 1 \leq n \leq k+1, \\
& \beta_{j_{n}, c}^{\mathcal{F}}=\frac{\alpha_{j_{n}}}{\alpha_{j_{n+1}}} \quad \text { for } \quad 1 \leq n \leq k+1,
\end{aligned}
$$

and

where we set $\alpha_{j_{k+2}}=1$; the map $\pi^{*}$ is obtained by writing the $\alpha$ 's in terms of the $\beta^{\mathcal{F}, c}$ 's in the previous equations, i.e.,

$$
\begin{aligned}
\pi^{*}\left(\alpha_{j_{n}}\right) & =\beta_{j_{n}}^{\mathcal{F}, c} \beta_{j_{n+1}}^{\mathcal{F}, c} \ldots \beta_{j_{k+1}}^{\mathcal{F}, c} \\
\pi^{*}\left(\alpha_{i}\right) & =\beta_{i}^{\mathcal{F}, c} \beta_{j_{n}}^{\mathcal{F}, c} \beta_{j_{n+1}, c}^{\mathcal{F}, c} \ldots \beta_{j_{k+1}}^{\mathcal{F}, c} \quad \text { for } i \in I_{n} \backslash\left(\left\{j_{n}\right\} \cup I_{n-1}\right) .
\end{aligned}
$$

The morphism $\pi$ restricts to an isomorphism between the open subsets defined by $\beta_{j_{n}}^{\mathcal{F}, c} \neq 0$ and $\alpha_{j_{n}} \neq 0$ for all $1 \leq n \leq k$. A simple example is given in $\$ 12.0 .2$.

By (5.8), the coordinate rings of the $\mathbb{A}^{\mathcal{F}, c}$ are contained in the fraction field of $\mathbb{P}^{S}$, and glue together to form a scheme $P^{B}$ with a morphism $\pi: P^{B} \rightarrow \mathbb{P}^{S}$ over Spec $\mathbb{Z}$. We claim that $P^{B}$ is indeed the space defined by blow-ups in the first paragraph, and that the $\beta_{j}^{\mathcal{F}, c}$ are local coordinates in the neighbourhood of a 'corner'

$$
(\mathcal{F}, c): \quad \bigcap_{i \in S \backslash\left\{j_{1}, \ldots, j_{k}\right\}} D_{i} \cap \bigcap_{1 \leq n \leq k} D_{I_{n}}
$$

where the trace of $D_{I_{n}}$ on the chart $\mathbb{A}^{\mathcal{F}, c}$ is given by $\beta_{j_{n}, c}^{\mathcal{F}}=0$ and $D_{i}$ by $\beta_{i}^{\mathcal{F}, c}=0$. These divisors are clearly normal crossing, and meet according to the rules described 
in the second half of theorem 5.1. For example, divisors of the form $D_{I}$ and $D_{J}$ with $I, J \in B$ only meet on such a chart if the sets $I, J$ fit into a flag $\mathcal{F}$, in which case $I \subset J$ or $J \subset I$; the remaining cases are left to the reader.

Next observe that the product structure of these divisors is clear from the structure of the coordinates (5.8) which give a canonical isomorphism:

$$
D_{I_{r}} \cap \mathbb{A}^{\mathcal{F}, c}=V\left(\beta_{j_{r}}^{\mathcal{F}, c}\right) \stackrel{\sim}{\longrightarrow} \mathbb{A}^{\mathcal{F}^{I_{r}}, c^{I_{r}}} \times \mathbb{A}^{\mathcal{F}_{I_{r}}, c_{I_{r}}}
$$

where $\mathcal{F}^{I_{r}}, \mathcal{F}_{I_{r}}$ are the flags in $I_{r}$ and $S \backslash I_{r}$ defined by

$$
\begin{array}{lll}
\mathcal{F}^{I_{r}} & : & \emptyset=I_{0} \subsetneq I_{1} \subsetneq I_{2} \ldots \subsetneq I_{r} \\
\mathcal{F}_{I_{r}}: & \emptyset \subsetneq I_{r+1} \backslash I_{r} \subsetneq \ldots \subsetneq I_{k} \backslash I_{r} \subsetneq S \backslash I_{r}
\end{array}
$$

and $c^{I_{r}}, c_{I_{r}}$ are the obvious restrictions of $c$ to $\mathcal{F}^{I_{k}}, \mathcal{F}_{I_{k}}$ respectively. Gluing the morphisms (5.10) together for all $\mathcal{F}, c$ proves the first equation of (5.5) since every pair of flags in $B^{I_{r}}, B_{I_{r}}$ is obtained from a flag in $B$ in this way. Similarly,

$$
\mathbb{A}^{\mathcal{F}, c} \supset V\left(\beta_{i}^{\mathcal{F}, c}\right) \stackrel{\sim}{\longrightarrow} \mathbb{A}^{\mathcal{F}_{i}, c_{i}}
$$

where $i \in I_{r} \backslash\left(I_{r-1} \cup\left\{j_{r}\right\}\right)$ and $\mathcal{F}_{i}$ is the flag in $S \backslash\{i\}$ defined by

$$
\mathcal{F}_{i}: \quad \emptyset=I_{0} \subsetneq I_{1} \subsetneq \ldots \subsetneq I_{r-1} \subsetneq I_{r} \backslash\{i\} \subsetneq \ldots \subsetneq I_{k} \backslash\{i\} \subsetneq I_{k+1}=S \backslash\{i\},
$$

which proves the second equation of (5.5). It remains to show that the spaces $P^{B}$ as defined above are indeed given by the blow-up procedure. This can be proved by induction. First of all, if $B$ is empty, then $P^{B}$ is simply $\mathbb{P}^{S}$ and the coordinate charts $\mathbb{A}^{\mathcal{F}, c}$ are the usual affine covering of $\mathbb{P}^{S}$. Let $P_{-1}=\mathbb{P}^{S}$, and suppose by induction that $P_{n-1}$ is isomorphic to $P^{B(n-1)}$ where $B(n-1)$ is the subset of $B$ consisting of all $I \in B$ such that $|I| \geq|S|-n$, where $n \geq 0$. It is stable under unions. Let $I \in B$ of cardinality $|I|=|S|-n-1$.

Lemma 5.4. Let $\mathcal{F}, c$ be a maximal flag in $B(n-1)$ given by (5.6) and (5.7) where $I_{1}, \ldots, I_{k} \in B(n-1)$. Let $I \in B(n)$ as above. The strict transform of $L_{I}$ in $P^{B(n-1)}$ meets the chart $\mathbb{A}^{\mathcal{F}, c}$ if and only if $I \subset I_{1} \backslash\left\{j_{1}\right\}$.

Proof. If $j_{r} \in I$ for any $1 \leq r \leq k+1$ then the strict transform of $L_{I}$ is contained in the strict transform of $\bar{L}_{j_{r}}$, which does not meet $\mathbb{A}^{\mathcal{F}, c}$ by inspection of the first equation of (5.9) (its total transform is contained in the union of the vanishing locus of the $\beta_{j_{k}}^{\mathcal{F}, c}$, which are all exceptional divisors). On the other hand, suppose that $I$ does not contain any $j_{r}$, and is not contained in $I_{1}$. Let $m$ be the smallest integer such that $I \subset I_{m+1}$. Then $I \subsetneq I_{m}$ so

$$
I_{m} \subsetneq I \cup I_{m} \subsetneq I_{m+1} \text {. }
$$

Since $B$ is closed under unions, $I \cup I_{m}$ is an element of $B(n-1)$ which contradicts the maximality of $\mathcal{F}, c\left(\right.$ as $\left.j_{m+1} \notin I\right)$. Thus for the strict transform of $L_{I}$ to meet $\mathbb{A}^{\mathcal{F}, c}$, we must have $I \subset I_{1}$ and $j_{1} \notin I$. Conversely, when this holds, the intersection of the strict transform of $L_{I}$ with $\mathbb{A}^{\mathcal{F}, c}$ is given by the equations $\beta_{i}^{\mathcal{F}, c}=0$ for $i \in I$.

Now, in the situation of the previous lemma, we can blow up the strict transform $L_{I}$ explicitly in the affine chart $\mathbb{A}^{\mathcal{F}, c}$ using example 5.3. It is covered by the affine charts $\mathbb{A}^{\mathcal{F}^{\prime}, c^{\prime}}$ where $\mathcal{F}^{\prime}, c^{\prime}$ extends $\mathcal{F}, c$ to the left:

$$
\mathcal{F}^{\prime}: \quad \emptyset \subsetneq I \subsetneq I_{1} \subseteq \ldots \subsetneq I_{k} \subsetneq S
$$

and the restriction of $c^{\prime}$ to $\mathcal{F}$ is $c$. Proceeding in this way, we can blow up the strict transforms of $L_{I}$, for $I \in B$ of cardinality $|I|=|S|-n-1$ in any order, to 
give exactly the space $P^{B(n)}$ together with its affine covering $\mathbb{A}^{\mathcal{F}^{\prime}, c}$. This proves by induction that the two descriptions of $P^{B}$, by explicit coordinates and also by iterated blow-ups, are one and the same.

5.4. An affine model. Given $B$ satisfying (5.1) with $S \in B$, we shall construct an affine subspace $A^{B} \subset P^{B}$ by removing strict transforms of certain linear hyperplanes, in such a way that the product-structure of $P^{B}$ is also satisfied by $A^{B}$.

We can assume that for every $I \in B,|I| \geq 2$. For any subset $J \subset S$, denote by

$$
\alpha_{J}=\sum_{j \in J} \alpha_{j}
$$

Let $H_{J}=V\left(\alpha_{J}\right) \subset \mathbb{P}^{S}$ denote the corresponding hyperplane. Write $\mathbb{A}^{S}=\mathbb{P}^{S} \backslash H_{S}$, and consider the hyperplane complement $\mathbb{A}^{S} \backslash \cup_{I \in B} H_{I}$. We shall directly define a partial compactification of this space as follows. Write $B^{+}=B \cup\{\{i\}, i \in S\}$.

Definition 5.5. Let $R_{B}=\mathbb{Z}\left[b_{I / J}: I \subset S, J \in B\right.$ such that $\left.\emptyset \neq I \subseteq J\right] / \mathcal{I}$, where $\mathcal{I}$ is the ideal generated by the following relations:

$$
\begin{aligned}
b_{I / J} & =\sum_{i \in I} b_{\{i\} / J} \\
b_{J / J} & =1 \\
b_{I / J} b_{J / K} & =b_{I / K} \quad \text { if } J, K \in B
\end{aligned}
$$

For every $I \in B^{+} \backslash\{S\}$, let $D_{I}=V\left(b_{I / J}\right.$ for all $\left.I \subsetneq J \in B\right) \subset \operatorname{Spec}\left(R_{B}\right)$.

There is a homomorphism

$$
\begin{aligned}
R_{B} & \longrightarrow \mathbb{Z}\left[\alpha_{i} \text { for } i \in S, \alpha_{J}^{-1} \text { for } J \in B,|J| \geq 2\right] \\
b_{I / J} & \mapsto \frac{\alpha_{I}}{\alpha_{J}}
\end{aligned}
$$

which respects the relations (5.11), and hence a morphism

$$
\mathbb{A}^{S} \backslash \cup_{I \in B} H_{I} \longrightarrow \operatorname{Spec}\left(R_{B}\right) \text {. }
$$

Let $\pi: P^{B} \rightarrow \mathbb{P}^{S}$ denote the blow-up defined in the previous paragraphs. Let $\widetilde{H}_{I}$ denote the strict transform of $H_{I}$, for $I \in B^{+}$.

Definition 5.6. Let $A^{B}=P^{B} \backslash \cup_{I \in B} \widetilde{H}_{I}$. For every $I \in B^{+} \backslash\{S\}$, let $D_{I} \subset P^{B}$ denote the divisor defined in $\S 5.3$, and, by abuse of notation, let us also denote by $D_{I}$ its intersection with $A^{B}$. Let $D$ denote their union.

Observe that $\mathbb{A}^{S} \backslash \cup_{I \in B} H_{I}$ is an open subspace of $A^{B}$, since the image in $\mathbb{P}^{S}$ of every exceptional divisor in $P^{B}$ is contained in some such hyperplane $H_{I}$. More precisely, $\mathbb{A}^{S} \backslash \cup_{I \in B} H_{I} \cong P^{B} \backslash\left(D \cup \cup_{I \in B} \widetilde{H}_{I}\right)$.

Theorem 5.7. The morphism (5.13) extends to a canonical isomorphism

$$
A^{B} \stackrel{\sim}{\longrightarrow} \operatorname{Spec} R_{B}
$$

In particular, (5.13) has Zariski-dense image, and furthermore, $A^{B}$ is affine and $\operatorname{Spec} R_{B}$ is smooth over $\mathbb{Z}$. The divisors denoted $D_{I}$ in $A^{B}$ and $\operatorname{Spec} R_{B}$ are mapped isomorphically to each other, and there are canonical isomorphisms

$$
D_{i}=\operatorname{Spec} \mathbb{Z} \times A^{B_{i}} \quad \text { and } \quad D_{I}=A^{B^{I}} \times A^{B_{I}} .
$$


If the set $S=\{1, \ldots, n\}$ is ordered, and $B$ consists of all consecutive sets $\{k, k+$ $1, \ldots, k+\ell\}$, for $1 \leq k \leq n-\ell$ and $\ell \geq 2$, then $A^{B}$ is isomorphic to the affine space $\mathfrak{M}_{0, n+2}^{\delta}$ defined in the author's thesis ${ }^{11}$. The strategy of $\S 5.5$ gives a new proof of its main properties. On the other hand, if we take $B$ to be all subsets of $S$ of cardinality $\geq 2$, we obtain an affine algebraic model of a permutohedron.

Example 5.8. Let $S=\{1,2\}$, and let $B=S$. Then there is nothing to blow up and $P^{B}=\mathbb{P}^{1}$. Its affine version is $A^{B}=\mathbb{P}^{1} \backslash\left\{\alpha_{1}+\alpha_{2}=0\right\} \cong \mathbb{A}^{1}$. In the explicit coordinates above, $R_{B}=\mathbb{Z}\left[b_{1 / 12}, b_{2 / 12}\right] / \mathcal{I}$ where $\mathcal{I}$ is the ideal generated by the relation $b_{1 / 12}+b_{2 / 12}=1$, and the composition with inclusion into $\mathbb{A}^{2}$

$$
A^{B} \stackrel{\sim}{\longrightarrow} \operatorname{Spec} R_{B} \subset \mathbb{A}^{2}
$$

is given in projective coordinates by $\left(\alpha_{1}: \alpha_{2}\right) \mapsto\left(\alpha_{1} /\left(\alpha_{1}+\alpha_{2}\right), \alpha_{2} /\left(\alpha_{1}+\alpha_{2}\right)\right)$.

5.5. Outline of proof of theorem 5.7. Let $(\mathcal{F}, c)$ denote a maximal pair $(5.6)$ and $\mathbb{A}^{\mathcal{F}, c}$ the corresponding chart of $P^{B}$. Let us denote by $U^{\mathcal{F}, c}$ the open $\mathbb{A}^{\mathcal{F}, c} \cap A^{B}$. We must first show that the morphism (5.13) canonically extends to a morphism

$$
U^{\mathcal{F}, c} \longrightarrow \operatorname{Spec} R_{B}
$$

To see this, compute the strict transform of $H_{J}$, for $J \in B$ in the coordinates $\beta_{i}^{\mathcal{F}, c}$. It follows from the definition of the coordinates $\beta^{\mathcal{F}, c}$ in (5.8) that

$$
\pi^{*} \alpha_{J}=P_{J} \times \prod_{r \geq \ell}^{k} \beta_{j_{r}}^{\mathcal{F}, c}
$$

where $P_{J}$ is an irreducible polynomial in the $\beta_{i}^{\mathcal{F}, c}$, and $\ell$ is the smallest integer such that $J \subseteq I_{\ell}$. We have $P_{\left\{j_{n}\right\}}=1$. The strict transform $\widetilde{H}_{J}$ of $H_{J}$ is given locally on $\mathbb{A}^{\mathcal{F}, c}$ by the zero locus of $P_{J}$. It follows from this calculation that

$$
\mathcal{O}\left(U^{\mathcal{F}, c}\right)=\operatorname{Spec}\left(\mathbb{Z}\left[\beta^{\mathcal{F}, c}\right]\left[P_{J}^{-1}, \text { for } J \in B,|J| \geq 2\right]\right)
$$

and that we have explicitly

$$
\begin{aligned}
R_{B} & \longrightarrow \mathcal{O}\left(U^{\mathcal{F}, c}\right) \\
b_{I / J} & \mapsto \frac{P_{I}}{P_{J}} \times \prod_{r=\ell_{1}}^{\ell_{2}-1} \beta_{j_{r}}^{\mathcal{F}, c}
\end{aligned}
$$

where $\ell_{1} \leq \ell_{2}$ are minimal such that $I \subset I_{\ell_{1}}, J \subset I_{\ell_{2}}$. The point is that there are no terms $\beta_{j_{r}}^{\mathcal{F}, c}$ in the denominator. One can check that (5.14) is well-defined (respects the relations in the defining ideal of $R_{B}$ ), since it is compatible with $\pi^{*}$ and (5.13). Gluing the resulting maps $U^{\mathcal{F}, c} \rightarrow \operatorname{Spec} R_{B}$ together, we deduce that (5.13) extends to a morphism

$$
A^{B}=\bigcup_{\mathcal{F}, c} U^{\mathcal{F}, c} \longrightarrow \operatorname{Spec} R_{B} .
$$

For the next step, observe that if $j_{r} \in J \subset I_{r}$ then by the maximality of $(\mathcal{F}, c)$ there is no smaller $\ell<r$ such that $J \subset I_{\ell}$ and therefore under (5.14)

$$
b_{J / I_{r}} \mapsto \frac{P_{J}}{P_{I_{r}}} .
$$

\footnotetext{
${ }^{11}$ To write down the isomorphism, it suffices to observe that the simplicial coordinates $t_{i}$ for $1 \leq i \leq n-3$ correspond to $b_{I / S}$ where $I=\{1,2, \ldots, i\}$ and $S=\{1,2, \ldots, n-2\}$.
} 
Setting $J=\left\{j_{r}\right\}$, we have $b_{j_{r} / I_{r}} \mapsto P_{I_{r}}^{-1}$, which is invertible in $\mathcal{O}\left(U^{\mathcal{F}, c}\right)$. It follows that (5.14) factorizes through the ring

$$
R_{B}\left[b_{j_{1} / I_{1}}^{-1}, \ldots, b_{j_{k+1} / I_{k+1}}^{-1}\right] \longrightarrow \mathcal{O}\left(U^{\mathcal{F}, c}\right)
$$

where $I_{k+1}=S$. Note that the defining relation $b_{j_{r} / I_{r}}=b_{j_{r} / J} b_{J / I_{r}}$ implies that inverting $b_{j_{r} / I_{r}}$ also inverts $b_{J / I_{r}}$ and $b_{j_{r} / J}$ for $J \in B$. We claim that (5.15) is an isomorphism. To see this, we can write down a map in the opposite direction

$$
\begin{aligned}
\beta_{i}^{\mathcal{F}, c} & \mapsto b_{i / I_{n}} b_{j_{n} / I_{n}}^{-1} \quad \text { if } i \in I_{n} \backslash\left(\left\{j_{n}\right\} \cup I_{n-1}\right), \quad 1 \leq n \leq k+1, \\
\text { and } \quad \beta_{j_{n}}^{\mathcal{F}, c} & \mapsto b_{j_{n} / I_{n+1}} b_{j_{n+1} / I_{n+1}}^{-1} \quad \text { for } \quad 1 \leq n \leq k+1,
\end{aligned}
$$

consistent with (5.8). It clearly lands in $R_{B}\left[b_{j_{1} / I_{1}}^{-1}, \ldots, b_{j_{k+1} / I_{k+1}}^{-1}\right]$, and one checks that it is indeed the inverse of (5.14). We omit the details.

For the final step, define open affine subspaces $V^{\mathcal{F}, c} \subset \operatorname{Spec} R_{B}$ by

$$
V^{\mathcal{F}, c}=\operatorname{Spec} R_{B}\left[b_{j_{1} / I_{1}}^{-1}, \ldots, b_{j_{k+1} / I_{k+1}}^{-1}\right] .
$$

We have shown that $U^{\mathcal{F}, c} \cong V^{\mathcal{F}, c}$ are canonically isomorphic. It suffices to check that the opens $V^{\mathcal{F}, c}$ form a covering of $\operatorname{Spec} R_{B}$. To see this, observe that

$$
\bigcup_{i \in I_{1}} \operatorname{Spec} R_{B}\left[b_{i / I_{1}}^{-1}, b_{j_{2} / I_{2}}^{-1}, \ldots, b_{j_{k+1} / I_{k+1}}^{-1}\right] \subseteq \operatorname{Spec} R_{B}\left[b_{j_{2} / I_{2}}^{-1}, \ldots, b_{j_{k+1} / I_{k+1}}^{-1}\right]
$$

is an equality, which follows from the partition of unity relation $\sum_{i \in I_{1}} b_{i / I_{1}}=1$ in $R_{B}$. By varying $c$ in $(\mathcal{F}, c)$, and eliminating the $b_{j_{r} / I_{r}}^{-1}$ in turn, we deduce that

$$
\bigcup_{(\mathcal{F}, c)} V^{\mathcal{F}, c}=\operatorname{Spec} R_{B}
$$

This proves that $A^{B} \stackrel{\sim}{\rightarrow} \operatorname{Spec} R_{B}$ is an isomorphism. The next statements follow by transferring information along this isomorphism: $\operatorname{Spec} R_{B}$ is clearly affine, and $A^{B}$, defined by the complement of divisors in a blow-up, is smooth over $\mathbb{Z}$.

For the last statement concerning the product structure of the divisors $D_{I}$, we refer to theorem 6.5. Note that $A^{B}=P^{B} \backslash Y$, where $Y$ is the strict transform of the zero locus of the polynomials $\phi_{B}(\underline{n})=\prod_{J \in B} \alpha_{J}^{n_{J}}$, where $\underline{n}=\left(n_{J}\right)_{J \in B}$ are integers $n_{J} \geq 0$. They satisfy the same factorisation properties as graph polynomials which are used in the proof of theorem 6.5, namely

$$
\phi_{B}(\underline{n})=\phi_{B^{I}}\left(\underline{n}^{\prime}\right) \phi_{B_{I}}\left(\underline{n}^{\prime \prime}\right)+R
$$

for every $I \in B$, where $R$ is of higher degree in the variables $\alpha_{i}, i \in I$ than $\phi_{B^{I}}$.

\section{Motives OF GRAPHS With Kinematics}

We define the motive (or rather, its image in a category of realisations) of a Feynman graph by applying the linear blow-up construction of the previous section to the set of motic subgraphs of a Feynman graph. In the case where the graph has no kinematic dependence, and is primitive log-divergent, this retrieves the definition of graph motive due to Bloch-Esnault-Kreimer [10]. 
6.1. Orders of vanishing. Let $G$ be a Feynman graph, and consider the projective space $\mathbb{P}^{E_{G}}$. There is a bijection between coordinate linear subspaces

$$
\mathbb{P}^{E_{G}} \supset L_{I} \text { for } I \subsetneq E_{G} \longleftrightarrow \text { edge subgraphs } I \subsetneq G \text {. }
$$

Given a homogenous polynomial $P$ in $\mathbb{Z}\left[\alpha_{e}, e \in E_{G}\right]$, let

$$
v_{I}(P)=\text { order of vanishing of } P \text { along } L_{I} \text {. }
$$

Lemma 6.1. Assume generic kinematics (1.17) and (1.18). Let $\gamma \subset E_{G}$. Then

$$
\begin{aligned}
& v_{\gamma}\left(\Psi_{G}\right)=h_{\gamma}, \\
& v_{\gamma}\left(\Xi_{G}\right)= \begin{cases}h_{\gamma}+1 & \text { if } \gamma \text { is m.m. } \\
h_{\gamma} & \text { if } \gamma \text { is not m.m. }\end{cases}
\end{aligned}
$$

Proof. This follows from the factorisations (2.6) and (2.7), the degree formulae for graph polynomials (1.8), and lemmas 1.11 and 1.13 which assert that $\Psi_{G / \gamma}$ and $\Xi_{G / \gamma}(m, q)$ are non-zero, via equation $(2.5)$.

The principle motivation for the definition of motic subgraphs comes from the following proposition. We again assume generic kinematics (1.17), (1.18).

Proposition 6.2. An edge subgraph $\Gamma \subset E_{G}$ is motic if and only if

$$
v_{\gamma}\left(\Xi_{G}\right)<v_{\Gamma}\left(\Xi_{G}\right)
$$

for all strict edge subgraphs $\gamma \subsetneq \Gamma$. In particular, $v_{\Gamma}\left(\Xi_{G}\right)>0$ if $\Gamma$ is motic.

Proof. Let $\gamma \subsetneq \Gamma$ be a strict edge subgraph. Let $\mathbb{I}_{\gamma, G}$ equal 1 if $\gamma$ is mass-momentum spanning in $G$ and 0 otherwise. We have $h_{\Gamma} \geq h_{\gamma}$.

Equation (6.2) implies that

$$
v_{\Gamma}\left(\Xi_{G}\right)-v_{\gamma}\left(\Xi_{G}\right)=\left(h_{\Gamma}-h_{\gamma}\right)+\left(\mathbb{I}_{\Gamma, G}-\mathbb{I}_{\gamma, G}\right) .
$$

Furthermore, $\mathbb{I}_{\Gamma, G} \geq \mathbb{I}_{\gamma, G}$, because if $\gamma$ is mass-momentum spanning in $G$, then so too is $\Gamma$ by lemma $3.4(i)$. Thus $(6.3)$ is strictly positive for all strict edge subgraphs $\gamma \subset \Gamma$ if and only if $h_{\gamma}<h_{\Gamma}$ whenever $\mathbb{I}_{\gamma, G}=\mathbb{I}_{\Gamma, G}$. By lemma $3.4(i)$, this is precisely the set of mass-momentum spanning subgraphs $\gamma \subsetneq \Gamma$.

6.2. Graph hypersurfaces and the motive. Let $G$ be a graph of type $(Q, M)$. We shall construct various families of schemes over the space of kinematics $K_{Q, M}$, defined in (1.20). In order to lighten the notation, we shall abusively write $\mathbb{P}^{E_{G}}$, $L_{I}$, and so on, to denote the base change of the schemes defined in the previous section from Spec $\mathbb{Z}$ to $K_{Q, M}$. Define graph hypersurfaces

$$
X_{\Psi_{G}}=V\left(\Psi_{G}\right) \subset \mathbb{P}^{E_{G}} \quad \text { and } \quad X_{\Xi_{G}(q, m)}=V\left(\Xi_{G}(q, m)\right) \subset \mathbb{P}^{E_{G}} .
$$

These are to be viewed as families of hypersurfaces over $K_{Q, M}$. The former were considered in [10] when $Q=M=0$. Note that the intersection $X_{\Psi_{G}} \cap X_{\Xi_{G}(q, m)}$ is given by the zero locus $V\left(\Phi_{G}(q)\right)$ of the second Symanzik polynomial, by (1.9).

If $\Gamma \subset G$ is motic, then by proposition $6.2, v_{\Gamma}\left(\Xi_{G}(q, m)\right)>0$ and so the linear subspace $L_{\Gamma}$ is contained in the graph hypersurface on each fiber, i.e.,

$$
L_{\Gamma} \subset X_{\Xi_{G}(q, m)} .
$$


If $\Gamma$ is not $m . m$. in $G$, then we also have $L_{\Gamma} \subset X_{\Psi_{G}}$ by (6.1) since $h_{\Gamma}>0$. Thus the loci $L_{\Gamma}$ meet both the boundary of the chain of integration $\sigma$, and the singularities of the Feynman integrand (1.11), causing potential divergences ${ }^{12}$.

Definition 6.3. Let $G$ be a motic Feynman graph of type $(Q, M)$. Define

$$
\pi_{G}: P^{G}=P^{B_{G}} \longrightarrow \mathbb{P}^{E_{G}}
$$

where $B_{G}=\{\Gamma \subsetneq G$ motic $\}$, which is stable under unions by theorem 3.6 (iii).

Let us define $X_{G} \subset \mathbb{P}^{E_{G}}$ (viewed as a family over $K_{Q, M}$ ) to be

$$
X_{G}=\left\{\begin{array}{ll}
X_{\Psi_{G}} \cup X_{\Xi_{G}(q, m)} & \text { if } G \text { has non-trivial kinematics } \\
X_{\Psi_{G}} & \text { if } G \text { has no masses or momenta }
\end{array} .\right.
$$

Because of exceptional cases when the Feynman integrand (1.11) has no term $\Psi_{G}$ in the denominator (i.e., when $\left.N_{G} \geq\left(h_{G}+1\right) d / 2\right)$ ), we can also define

$$
X_{G}^{\prime}=X_{\Xi_{G}(q, m)} \text {. }
$$

Denote the strict transforms of $X_{\Psi_{G}}, X_{\Xi_{G}(q, m)}, X_{G}, X_{G}^{\prime}$ in $P^{G}$ by

$$
Y_{\Psi_{G}}, Y_{\Xi_{G}(q, m)}, Y_{G}, Y_{G}^{\prime}
$$

respectively. Note that if $G$ has connected components $G_{1}, \ldots, G_{n}$, then $Y_{\Psi_{G}}=$ $\bigcup_{i=1}^{n} Y_{\Psi_{G_{i}}}$. Recall that $D \subset P^{G}$ is the divisor defined by (5.2), base-changed from Spec $\mathbb{Z}$ to $K_{Q, M}$. Denote the canonical projection by $\pi_{G}: P^{G} \rightarrow \mathbb{P}^{E_{G}}$.

Definition 6.4. Let $G$ be a motic Feynman graph of type $(Q, M)$. Let

$$
\operatorname{mot}_{G}=H^{N_{G}-1}\left(P^{G} \backslash Y_{G}, D \backslash\left(D \cap Y_{G}\right)\right)_{/ S} .
$$

It is a triple $\operatorname{mot}_{G}=\left(\left(\operatorname{mot}_{G}\right)_{B},\left(\operatorname{mot}_{G}\right)_{d R}, c\right)$ in a category $\mathcal{H}(S)$, for some Zariskiopen $S \subset K_{Q, M}$, defined immediately below, via the construction of [17] $\$ 10.2$. There is also a variant

$$
\operatorname{mot}_{G}^{\prime}=H^{N_{G}-1}\left(P^{G} \backslash Y_{G}^{\prime}, D \backslash\left(D \cap Y_{G}^{\prime}\right)\right)_{/ S} .
$$

6.3. Reminders from [17]. Let $S$ be a smooth geometrically connected scheme over $\mathbb{Q}$. Then $\mathcal{H}(S)$ is the category of triples $\left(\mathcal{V}_{B}, \mathcal{V}_{d R}, c\right)$ where $\mathcal{V}_{B}$ is a local system of $\mathbb{Q}$-vector spaces on $S(\mathbb{C})$, and $\mathcal{V}_{d R}$ is an algebraic vector bundle on $S$ equipped with an integrable connection $\nabla$ with regular singularities at infinity. These are equipped with a weight (and for $\mathcal{V}_{d R}$, a Hodge) filtration satisfying the conditions of $\S 7.2$ of [17], and $c: \mathcal{V}_{d R}^{\text {an }} \stackrel{\sim}{\rightarrow} \mathcal{V}_{B} \otimes_{\mathbb{Q}} \mathcal{O}_{S}$ an is an isomorphism of analytic vector bundles with connection, respecting the weight filtrations.

6.4. Recursive structure. Let $D \subset P^{G}$ denote the divisor defined by (5.2). Its irreducible components are $D_{e}$, for $e \in E_{G}$ an edge, and $D_{\gamma}$ for $\gamma \subsetneq E_{G}$ a strict motic subgraph of $G$. All schemes are viewed over $K_{Q, M}$ where $G$ is of type $(Q, M)$.

Theorem 6.5. For every edge $e \in E_{G}$ and $\gamma \subsetneq E_{G}$ motic, we have

$$
\begin{aligned}
& D_{\gamma}=P^{\gamma} \times P^{G / \gamma}, \\
& D_{e}=\{p t\} \times P^{G / e}
\end{aligned}
$$

\footnotetext{
${ }^{12}$ As suggested by T. Damour, such loci could be termed problemotic.
} 
and the strict transforms of the graph hypersurfaces satisfy:

$$
\begin{aligned}
& Y_{G} \cap D_{\gamma}=\left(Y_{\gamma} \times P^{G / \gamma}\right) \cup\left(P^{\gamma} \times Y_{G / \gamma}\right), \\
& Y_{G} \cap D_{e}=\{p t\} \times Y_{G / e} .
\end{aligned}
$$

Note that at most one of $\gamma$ and $G / \gamma$ has non-trivial momenta and masses. Here, $\{p t\}$ means the family of points $\operatorname{Spec} \mathbb{Z} \times$ Spec $\mathbb{Z} \operatorname{Spec} K_{Q, M}$ over $\operatorname{Spec} K_{Q, M}$.

Proof. Recall that $P^{G}=P^{B_{G}}$, where $B_{G}$ consists of the set of strict motic subgraphs of $G$. Let $\gamma \subsetneq E_{G}$ be motic. By theorem 5.1, and the notation preceding it,

$$
D_{\gamma}=P^{\left(B_{G}\right)^{\gamma}} \times P^{\left(B_{G}\right)_{\gamma}} .
$$

By remark 3.5, $\left(B_{G}\right)^{\gamma}=B_{\gamma}$ since a subgraph of $\gamma$ is motic if and only if it is motic in $G$. Finally, $\left(B_{G}\right)_{\gamma}=B_{G / \gamma}$ by theorem $3.6(i)$ and $(i i)$. This proves the first line of (6.6). Now consider an affine chart $\mathbb{A}^{\mathcal{F}, c}$ defined in $\S 5.3$, where the flag $\mathcal{F}$ contains $\gamma$ (otherwise $D_{\gamma}$ does not meet $\mathbb{A}^{\mathcal{F}, c}$, by lemma 5.4 ). In the coordinates (5.8), let $\beta=0$ denote the equation of $D_{\gamma} \cap \mathbb{A}^{\mathcal{F}, c}$ in $\mathbb{A}^{\mathcal{F}, c}$. Write $\pi_{G}^{*} \Psi_{G}$ in these coordinates and apply (2.4) to obtain

$$
\pi_{G}^{*} \Psi_{G}=\beta^{h_{\gamma}}\left(\pi_{\gamma}^{*} \Psi_{\gamma}\right)\left(\pi_{G / \gamma}^{*} \Psi_{G / \gamma}\right)+O\left(\beta^{h_{\gamma}+1}\right),
$$

where the right-hand side is written using $\mathbb{A}^{\mathcal{F}, c} \cong \mathbb{A}^{1} \times \mathbb{A}^{\mathcal{F}^{\gamma}, c^{\gamma}} \times \mathbb{A}^{\mathcal{F}_{\gamma}, c_{\gamma}}$ and where the coordinate on the component $\mathbb{A}^{1}$ is $\beta$ (see the discussion following (5.10)). This proves that $Y_{\Psi_{G}} \cap D_{\gamma}=\left(Y_{\Psi_{\gamma}} \times P^{G / \gamma}\right) \cup\left(P^{\gamma} \times Y_{\Psi_{G / \gamma}}\right)$. A similar argument using formulae (2.6) and (2.7) proves that

$$
\begin{aligned}
& Y_{\Xi_{G}(q, m)} \cap D_{\gamma}=\left(Y_{\Xi_{\gamma}(q, m)} \times P^{G / \gamma}\right) \cup\left(P^{\gamma} \times Y_{\Psi_{G / \gamma}}\right) \quad \text { when } \gamma \text { is } m . m . \\
& Y_{\Xi_{G}(q, m)} \cap D_{\gamma}=\left(Y_{\Psi_{\gamma}} \times P^{G / \gamma}\right) \cup\left(P^{\gamma} \times Y_{\Xi_{G / \gamma}(q, m)}\right) \quad \gamma \text { not m.m. }
\end{aligned}
$$

Since $Y_{G}=Y_{\Psi_{G}} \cup Y_{\Xi_{G}(q, m)}$, this proves the first equation of (6.7).

Now, by theorem 5.1, we have

$$
D_{e}=\{p t\} \times P^{\left(B_{G}\right)_{e}} .
$$

We must show that $\left(B_{G}\right)_{e}=B_{G / e}$. The left-hand side is given by the sets of edges $\gamma \backslash(\gamma \cap\{e\})$ for motic $\gamma \subsetneq E_{G}$, and this is the set of edges of the subgraph $(\gamma \cup e) / e \subset E_{G / e}$. By theorem 3.6 (i) and $(i v)$, such a graph is motic in $G / e$, and every motic subgraph of $G / e$ arises in this way. (Note that the failure of injectivity stated in the last line of theorem 3.6 (iv) poses no problem, since we are only concerned with $\gamma \cup e$ ). This proves the second line of (6.6). If $e$ is not a tadpole, it follows from corollary 1.10 that

$$
X_{\Psi_{G}} \cap L_{e}=X_{\Psi_{G / e}} \quad \text { and } \quad X_{\Xi_{G}(q, m)} \cap L_{e}=X_{\Xi_{G / e}(q, m)},
$$

and we deduce the corresponding statements for their strict transforms. If $e$ is a tadpole it is motic, and we are reduced to the previous case, since $P^{e}=\{p t\}$.

Remark 6.6. The analogous statement of theorem 6.5 for $Y_{G}^{\prime}=Y_{\Xi_{G}(q, m)}$ is

$$
\begin{aligned}
Y_{G}^{\prime} \cap D_{e} & =\{p t\} \times Y_{G / e}^{\prime} \\
Y_{G}^{\prime} \cap D_{\gamma} & =\left(Y_{\gamma}^{\prime} \times P^{G / \gamma}\right) \cup\left(P^{\gamma} \times Y_{G / \gamma}\right) \text { if } \gamma \text { is m.m. } \\
& =\left(Y_{\gamma} \times P^{G / \gamma}\right) \cup\left(P^{\gamma} \times Y_{G / \gamma}^{\prime}\right) \text { if } \gamma \text { is not m.m. }
\end{aligned}
$$

Note that every time a $Y_{H}$ (with no prime) occurs in these formulae, it is because the graph $H$ (either $\gamma$ or $G / \gamma$ ) has no masses or momentum dependence. 
By induction, the theorem implies that the intersection of the strict transform $Y_{G}$ of the graph hypersurface with a facet of $D$ of codimension $n$ is of the form

$$
\bigcup_{i} P^{\gamma_{1}} \times \ldots \times P^{\gamma_{i-1}} \times Y_{\gamma_{i}} \times P^{\gamma_{i+1}} \times \ldots \times P^{\gamma_{n}}
$$

where the $\gamma_{i}$ are quotients of motic subgraphs of $G$. In fact, $\gamma_{1} \otimes \ldots \otimes \gamma_{n}$ is a descendant of $G$ according to definition 4.5.

6.5. Feynman polytope and Betti class. Let $G$ be a Feynman graph of type $(Q, M)$. Define the Feynman polytope, following $§ 5.2$, to be

$$
\widetilde{\sigma}_{G}:=\widetilde{\sigma}_{B_{G}} \times U_{Q, M}^{\text {gen }} \subset P^{G}(\mathbb{R}) .
$$

It is a constant family of compact manifolds with corners over the locus $U_{Q, M}^{\text {gen }} \subset$ $K_{Q, M}^{g e n}(\mathbb{C})$ where masses and momenta have positive real parts $(\S 1.7)$.

Theorem 6.7. We have $\widetilde{\sigma}_{G} \cap Y_{G}(\mathbb{C})=\emptyset$. A fortiori, $\widetilde{\sigma}_{G} \cap Y_{G}^{\prime}(\mathbb{C})=\emptyset$.

Proof. Since the polytope $\widetilde{\sigma}_{G}$ is stratified, it suffices to show that $Y_{G}(\mathbb{C})$ does not meet any open stratum. We shall do this by induction. We have an isomorphism

$$
\left(P^{G} \backslash D\right) \stackrel{\sim}{\longrightarrow}\left(\mathbb{P}^{E_{G}} \backslash \bigcup_{e \in E_{G}} L_{e}\right)
$$

which induces a homeomorphism from the big open stratum $\stackrel{\circ}{\sigma}_{G} \subset \widetilde{\sigma}_{G}$ to $\stackrel{\circ}{\sigma} \times U_{Q, M}^{\text {gen }}$, the open coordinate simplex. It also sends $Y_{G} \backslash\left(Y_{G} \cap D\right)$ to $X_{G}$. Thus

$$
Y_{G}(\mathbb{C}) \cap \stackrel{\circ}{\sigma}_{G} \cong X_{G}(\mathbb{C}) \cap\left(\stackrel{\circ}{\sigma} \times U_{Q, M}^{\text {gen }}\right),
$$

and it suffices to show that $X_{\Psi_{G}}(\mathbb{C}) \cap\left(\stackrel{\circ}{\sigma} \times U_{Q, M}^{\text {gen }}\right)$ is empty, and likewise that $X_{\Xi_{G}(q, m)}(\mathbb{C}) \cap\left(\stackrel{\circ}{\sigma} \times U_{Q, M}^{\text {gen }}\right)$ is non-empty, in the case when $G$ has non-trivial kinematics. Since $\Psi_{G}$ is non-zero by lemma 1.11 and only has positive coefficients, it is clear that $\Psi_{G}>0$ when all $\alpha_{i}>0$. Similarly, if $G$ has non-trivial kinematics then $\Xi_{G}(q, m)$ is non-zero by lemma 1.13 since (1.17) and (1.18) is automatically satisfied if (1.22) holds. Using the explicit expression (1.9), we have $\operatorname{Re}\left(\Xi_{G}(q, m)\right)>0$ when all $\alpha_{i}>0$ and $(q, m) \in U_{Q, M}^{\text {gen }}$. This proves that $X_{G}(\mathbb{C}) \cap\left(\stackrel{\circ}{\sigma} \times U_{Q, M}^{\text {gen }}\right)=\emptyset$. Now theorem 6.5 implies that the facets of $\widetilde{\sigma}_{G}$ satisfy

$$
\tilde{\sigma}_{G} \cap D_{e}(\mathbb{C})=\{p t\} \times \widetilde{\sigma}_{G / e} \quad \text { and } \quad \tilde{\sigma}_{G} \cap D_{\gamma}(\mathbb{C})=\tilde{\sigma}_{\gamma} \times \widetilde{\sigma}_{G / \gamma} .
$$

Using the recursive structure (6.7), we are reduced to proving an identical statement for quotients of motic subgraphs of $G$, and proceed by induction by decreasing dimension of the strata.

Definition 6.8. Let us write $\sigma_{G} \subset\left(P^{G} \backslash Y_{G}\right)(\mathbb{R})$ (with no tilde) for the intersection

$$
\sigma_{G}=\widetilde{\sigma}_{G} \cap\left(P^{G} \backslash Y_{G}\right)(\mathbb{C}) .
$$

It follows from the previous theorem that $\sigma_{G}$ is homeomorphic to $\tilde{\sigma}_{G}$, and its boundary is contained in $D \cap Y_{G}(\mathbb{C})$. It therefore defines a canonical Betti class

$$
\left[\sigma_{G}\right] \in \Gamma\left(U_{Q, M}^{\text {gen }}, H_{N_{G}-1}\left(P^{G} \backslash Y_{G}, D \backslash\left(D \cap Y_{G}\right)\right)\right) .
$$

which we view as a local section of the dual Betti local system

$$
\left[\sigma_{G}\right] \in \Gamma\left(U_{Q, M}^{\text {gen }},\left(\operatorname{mot}_{G}\right)_{B}^{\vee}\right) \text {. }
$$

Likewise we can replace $Y_{G}$ by $Y_{G}^{\prime}$ in the case when $N_{G} \geq\left(h_{G}+1\right) d / 2$. 
One reason why we emphasise the action of the de Rham Galois group (as opposed to the Betti Galois group) on motivic Feynman integrals is because the Betti class is uniformly defined for all graphs and we wish to keep it fixed. Furthermore, we prefer to compute with differential forms rather than homology cycles.

6.6. De Rham class and power-counting. Let $G$ be as above. The pull-back of the form (1.11) along the map $\pi_{G}: P^{G} \backslash D \backslash Y_{G} \longrightarrow \mathbb{P}^{E_{G}} \backslash X_{G}$

$$
\pi_{G}^{*}\left(\omega_{G}(q, m)\right) \in \Omega^{N_{G}-1}\left(P^{G} \backslash D \backslash Y_{G} ; k_{Q, M}\right)
$$

may have poles along the exceptional divisors $D_{\gamma} \subset P^{G}$ for $\gamma$ motic.

Definition 6.9. The superficial degree of divergence of a graph $G$ is

$$
\operatorname{sd}_{G}=d h_{G} / 2-N_{G} .
$$

This is an integer, since $d$, the dimension of spacetime, was assumed to be even.

The following lemma gives necessary and sufficient conditions for the convergence of the Feynman integral in the enlarged Euclidean region in terms of the superficial degrees of divergences of motic subgraphs.

Lemma 6.10. Let $\gamma \subsetneq E_{G}$ be motic. Then $\pi_{G}^{*}\left(\omega_{G}(q, m)\right)$ has a pole along $D_{\gamma}$ of order given by the following formula:

$$
-v_{\gamma}\left(\pi_{G}^{*}\left(\omega_{G}(q, m)\right)\right)= \begin{cases}1+\mathrm{sd}_{\gamma} & \text { if } \gamma \text { is not m.m. } \\ 1+\mathrm{sd}_{\gamma}-\mathrm{sd}_{G} & \text { if } \gamma \text { is m.m. }\end{cases}
$$

It has no poles along any divisors of the form $D_{e}$, where $e \in E_{G}$ is an edge which is not motic. It follows that the Feynman integral $I_{G}(q, m)(1.10)$ is absolutely convergent in the region $U_{Q, M}^{\text {gen }}$ if and only if

$$
\begin{array}{ll}
\operatorname{sd}_{\gamma}<0 & \text { for all } \gamma \subsetneq E_{G} \text { motic and not m.m. } \\
\operatorname{sd}_{\gamma}<\operatorname{sd}_{G} & \text { for all } \gamma \subsetneq E_{G} \text { motic and m.m. . }
\end{array}
$$

Proof. Recall (1.11). From equations (6.2) and (6.1), we have

$$
-v_{\gamma}\left(\frac{1}{\Psi_{G}^{d / 2}}\left(\frac{\Psi_{G}}{\Xi_{G}(m, q)}\right)^{N_{G}-h_{G} d / 2}\right)=h_{\gamma} d / 2-\mathbb{I}_{\gamma} \operatorname{sd}_{G}
$$

where $\mathbb{I}_{\gamma}$ is 1 if $\gamma$ is m.m., and 0 otherwise. Now consider an affine chart $\mathbb{A}^{\mathcal{F}, c}$ where

$$
\mathcal{F}: \quad \emptyset=I_{0} \subsetneq I_{1} \subsetneq I_{2} \subsetneq \ldots \subsetneq I_{k} \subsetneq I_{k+1}=E_{G}
$$

is a flag containing $\gamma=I_{r}$ and $D_{\gamma}$ is given by the equation $\beta_{j_{r}}=0$. The chart $\mathbb{A}^{\mathcal{F}, c}$ lies over the affine $\alpha_{j_{k+1}}=1$. In the local coordinates (5.8) we find that

$$
\pi_{G}^{*}\left(\prod_{i \neq j_{k+1}} d \alpha_{i}\right)=\beta_{j_{1}}^{\left|I_{1}\right|-1} \beta_{j_{2}}^{\left|I_{2}\right|-1 \mid} \ldots \beta_{j_{k}}^{\left|I_{k}\right|-1} \prod_{i \neq j_{k+1}} d \beta_{i}
$$

vanishes along $\beta_{j_{r}}=0$ to order $1-\left|I_{r}\right|=1-N_{\gamma}$. Thus

$$
-v_{\gamma}\left(\pi^{*}\left(\omega_{G}(q, m)\right)\right)=1-N_{\gamma}+h_{\gamma} d / 2-\mathbb{I}_{\gamma} \operatorname{sd}_{G}
$$

which proves (6.11). For the second part, observe that $\Psi_{G}$ and $\Xi_{G}(q, m)$ vanish along a coordinate hyperplane $L_{e}$ if and only if $e$ is motic. Therefore $\omega_{G}(q, m)$ has no pole along $D_{e}$ if $e$ is not motic.

For the last part, suppose that $(6.12)$ holds. Then $\omega_{G}(q, m)$ is continuous and has no poles on the domain $\sigma_{G}$, which has compact fibers over $U_{Q, M}^{\text {gen }}$. Its integral 
$I_{G}(q, m)$ is therefore absolutely convergent. Conversely, suppose that $I_{G}(q, m)$ is absolutely convergent for some $(q, m)$ in the Euclidean region $K_{Q, M}^{\mathrm{gen}}(\mathbb{R})$ defined in $\S 1.7$, where all momenta $q_{i}$ and masses $m_{e}$ are real. Then $\omega_{G}(q, m)$ is strictly positive on $\sigma_{G}$, and so for any subset $U \subset \sigma_{G}$ we have

$$
\int_{U} \omega_{G}(q, m) \leq I_{G}(q, m)<\infty .
$$

If $\omega_{G}(q, m)$ had a pole along a boundary divisor $D_{\gamma}$, it follows from positivity by taking $U$ to be a neighbourhood of $D_{\gamma} \cap \sigma_{G}$ that the left-hand side is infinite. Therefore $\omega_{G}(q, m)$ has no poles along any $D_{\gamma}$ and hence (6.12) holds.

Note that one can interpret the quantity $\operatorname{sd}_{\gamma}-\operatorname{sd}_{G}$ as $-\operatorname{sd}_{G / \gamma}$. We say that a Feyman integral is convergent if the conditions (6.12) are satisfied. In this case,

$$
\pi_{G}^{*} \omega_{G}(q, m) \in \Omega^{N_{G}-1}\left(\left(P^{G} \backslash Y_{G}\right)_{*} ; k_{Q, M}\right)
$$

where $\left(P^{G} \backslash Y_{G}\right)_{*}$ denotes the generic fiber of $P^{G} \backslash Y_{G}$ over $K_{Q, M}$. This in turn defines a relative de Rham cohomology class at the generic point

$$
\left[\pi_{G}^{*} \omega_{G}(q, m)\right] \in \Gamma\left(\operatorname{Spec} k_{Q, M},\left(\operatorname{mot}_{G}\right)_{d R}\right) .
$$

In the case when $N_{G} \geq\left(h_{G}+1\right) d / 2$, we can replace $Y_{G}$ by $Y_{G}^{\prime}$ in the above.

Remark 6.11. The formulae for the degrees of divergence of a Feynman integral with respect to a subgraph are due to Weinberg [54], and are known as powercounting. Finding a minimal class of subgraphs which give necessary conditions for convergence is more subtle since the inequalities (6.12) are not independent. This problem was studied by Speer [51, 52] for generic Feynman integrals.

6.7. Convergence of 'global periods': integrals with numerators. Gauge theories can produce Feynman integrals with numerators [41]:

$$
\int_{\sigma} \omega(q, m) \quad \text { where } \quad \omega(q, m)=\frac{P \Omega_{G}}{\Psi_{G}^{A} \Xi_{G}(q, m)^{B}},
$$

where $A, B$ are integers, and $P \in \mathbb{Q}\left[\alpha_{e}, e \in E_{G}\right]$ is homogeneous of degree

$$
\operatorname{deg} P=A h_{G}+B\left(h_{G}+1\right)-N_{G}
$$

to ensure that $\omega(q, m)$ is homogeneous of degree zero. We take $B=0$ if $G$ is of type $(0,0)$, in which case $\Xi_{G}(q, m)$ vanishes.

Lemma 6.12. Suppose that

$$
v_{\gamma}(P) \geq A h_{\gamma}+B\left(h_{\gamma}+\mathbb{I}_{\gamma}\right)-N_{\gamma}+1
$$

for all motic subgraphs $\gamma \subsetneq E_{G}$, where $\mathbb{I}_{\gamma}$ is 0 if $\gamma$ is not m.m., and 1 if it is m.m.. Then $\pi_{G}^{*}(\omega(q, m))$ has no poles along $D$ for $(q, m) \in U_{Q, M}^{\text {gen }}$. Therefore

$$
\pi_{G}^{*}(\omega(q, m)) \in \Omega^{N_{G}-1}\left(P_{G} \backslash Y_{G} ; k_{Q, M}\right)
$$

and the integral (6.13) is convergent for all $(q, m) \in U_{Q, M}^{\mathrm{gen}}$.

Proof. Similar to lemma 6.10.

Thus in this case also $\left[\pi_{G}^{*} \omega_{G}(q, m)\right] \in \Gamma\left(\operatorname{Spec} k_{Q, M},\left(\operatorname{mot}_{G}\right)_{d R}\right)$. The integral (6.13) is an example of what we shall call a Feynman period. 
Example 6.13. Consider the banana graph $G$ with three edges, no external momenta and no masses. Its graph polynomial is $\Psi_{G}=\alpha_{1} \alpha_{2}+\alpha_{1} \alpha_{3}+\alpha_{2} \alpha_{3}$, and its Feynman amplitude is divergent. However, the previous lemma provides examples of Feynman periods such as

$$
\int_{\sigma} \frac{\alpha_{1} \alpha_{2} \alpha_{3}}{\Psi_{G}^{3}} \Omega_{G}=\int_{\alpha_{1}, \alpha_{2} \geq 0} \frac{\alpha_{1} \alpha_{2}}{\left(\alpha_{1} \alpha_{2}+\alpha_{1}+\alpha_{2}\right)^{3}}=\frac{1}{2} .
$$

One easily shows (see section $§ 9$ ) that all periods of this graph are rational.

\section{Motivic Feynman amplitudes}

Armed with the definition of the motive of a Feynman graph, we can give the definition of the motivic Feynman amplitude and draw some first consequences.

7.1. Reminders on motivic periods from [17]. Let $S \subset K_{Q, M}$ be Zariski open as in $\S 6.3$, let $s \in S(\mathbb{C})$, and denote by Spec $k_{Q, M}$ the generic point of $S$.

Then $\mathcal{H}(S)$ has two fiber functors ([17] $\$ 7.2 .1)$

$$
\begin{aligned}
\omega_{d R}^{\text {gen }}: \mathcal{H}(S) & \longrightarrow \operatorname{Vec}_{k_{Q}, M} \\
\omega_{B}^{s}: \mathcal{H}(S) & \longrightarrow \operatorname{Vec}_{\mathbb{Q}}
\end{aligned}
$$

where $\omega_{d R}^{\text {gen }}$ is the fiber of $\mathcal{V}_{d R}$ at the generic point $\operatorname{Spec} k_{Q, M}$ of $K_{Q, M}$, and $\omega_{B, s}\left(\mathcal{V}_{B}, \mathcal{V}_{d R}, c\right)=\left(\mathcal{V}_{B}\right)_{s}$ is the fiber at $s$. The ring of $\mathcal{H}(S)$-periods

$$
\mathcal{P}_{\mathcal{H}(S)}^{\mathfrak{m}, s, \text { gen }}
$$

is the space generated by the matrix coefficients $([17], \S 2.2, \S 8.2)$ of the form $[V, \sigma, \omega]^{\mathfrak{m}}$ where $V=\left(V_{B}, V_{d R}, c\right)$ is an object of $\mathcal{H}(S), \sigma \in \omega_{s}\left(V_{B}\right)^{\vee}$, and $\omega \in$ $\omega_{d R}^{\text {gen }}(V)$. Now suppose that $s_{1}, s_{2} \in U_{Q, M}^{\text {gen }} \cap S(\mathbb{C})$ are two points in the region of generic kinematics. A path $\gamma \subset U_{Q, M}^{\text {gen }} \cap S(\mathbb{C})$ from $s_{1}$ to $s_{2}$ yields an isomorphism

$$
\mathcal{P}_{\mathcal{H}(S)}^{\mathfrak{m}, s_{1}, \text { gen }} \cong \mathcal{P}_{\mathcal{H}(S)}^{\mathfrak{m}, s_{2}, \text { gen }}
$$

by continuation along paths [17], (7.10) and hence a canonical isomorphism

$$
\left(\mathcal{P}_{\mathcal{H}(S)}^{\mathfrak{m}, s_{1}, \text { gen }}\right)^{\pi_{1}\left(U_{Q, M}^{\text {gen }} \cap S(\mathbb{C}), s_{1}\right)}=\left(\mathcal{P}_{\mathcal{H}(S)}^{\mathfrak{m}, s_{2}, \text { gen }}\right)^{\pi_{1}\left(U_{Q, M}^{\text {gen }} \cap S(\mathbb{C}), s_{2}\right)}
$$

where the action of the topological fundamental group is on the right, and commutes with the action of the de Rham Tannaka group (resp. the de Rham coaction). If $S^{\prime} \rightarrow S$ is a smooth morphism and $s^{\prime} \in S^{\prime}(\mathbb{C})$ is in the pre-image of $s$, then the pullback defines a functor $\mathcal{H}(S) \rightarrow \mathcal{H}\left(S^{\prime}\right)$ and hence a homomorphism $\mathcal{P}_{\mathcal{H}(S)}^{\mathfrak{m}, s, \text { gen }} \rightarrow$ $\mathcal{P}_{\mathcal{H}\left(S^{\prime}\right)}^{\mathfrak{m}, s^{\prime} \text {,gen }}$. By taking $\pi_{1}$-invariants, we can move the complex point $s \in S(\mathbb{C})$ to ensure that it lies in the image of $S^{\prime}(\mathbb{C})$, and hence take the limit.

Definition 7.1. For $Q, M \geq 0$, following [17] $§ 8.2$, let

$$
\mathcal{P}_{Q, M}^{\mathfrak{m}}=\underset{S}{\lim }\left(\mathcal{P}_{\mathcal{H}(S)}^{\mathfrak{m}, s, \text { gen }}\right)^{\pi_{1}\left(U_{Q, M}^{\text {gen }} \cap S(\mathbb{C}), s\right)}
$$

where the limit ranges over Zariski open $S \subset K_{Q, M}^{\text {gen }}$ which are defined over $\mathbb{Q}$ and $s \in U_{Q, M}^{\text {gen }} \cap S(\mathbb{C})$, ordered with respect to inclusion.

The point of this construction is that the Feynman motivic periods will have poles on the complement of some unspecified Zariski-open set $S$, but will always be single-valued on the region $U_{Q, M}^{\text {gen }}$ (extended 'Euclidean sheet'). The ring $\mathcal{P}_{Q, M}^{\mathfrak{m}}$ captures exactly this property. 
There is a corresponding ring of de Rham periods $\mathcal{P}_{Q, M}^{\mathfrak{d r} r}=\lim _{\longrightarrow} \mathcal{P}_{\mathcal{H}(S)}^{\mathfrak{d r} \text {,gen }}$. It is generated by equivalence classes of triples $[V, f, \omega]^{\mathfrak{d r}}$ where $f \in \omega_{d R}^{\text {gen }}(V)^{\vee}$ and $\omega \in \omega_{d R}^{\text {gen }}(V)$ and $V$ is an object of $\mathcal{H}(S)$ for some $S \subset K_{Q, M}$ as above.

Recall that for $(Q, M)=(0,0)$ the ring $k_{0,0}=\mathbb{Q}$ and $\mathcal{H}_{0,0}=\mathcal{H}$ is the category of realisations over $\mathbb{Q}$ considered in [17] $\S 2$. Its ring of periods $\mathcal{P}_{0,0}^{\mathfrak{m}}=\mathcal{P}_{\mathcal{H}}^{\mathfrak{m}}$ is simply the ring of periods over $\mathbb{Q}$ considered in $[17] \S 3$.

7.2. Motivic Feynman amplitudes and motivic Feynman periods. We make frequent use of the definitions and constructions from [17].

Definition 7.2. Let $G$ be a Feynman graph of type $(Q, M)$ and $I_{G}(q, m)$ a convergent Feynman amplitude (1.10). Define the motivic Feynman amplitude to be

$$
I_{G}^{\mathfrak{m}}(q, m)=\left[\operatorname{mot}_{G},\left[\pi_{G}^{*} \omega_{G}(q, m)\right],\left[\sigma_{G}\right]\right]^{\mathfrak{m}} \quad \in \quad \mathcal{P}_{Q, M}^{\mathfrak{m}} .
$$

To check that this makes sense, note that the general theory implies that $\operatorname{mot}_{G}$ is an object of $\mathcal{H}(S)$ for $S$ some Zariski-open set in $K_{Q, M}^{\text {gen }}$. Since $\left[\sigma_{G}\right]$ is in a fact constant section of $\Gamma\left(U_{Q, M}^{\text {gen }} \cap S(\mathbb{C}),\left(\operatorname{mot}_{G}\right)_{B}^{\vee}\right)$, the fundamental group of $U_{Q, M}^{\text {gen }} \cap S(\mathbb{C})$ acts trivially upon $I_{G}^{\mathfrak{m}}(q, m)$. Pick any $s \in U_{Q, M}^{\text {gen }} \cap S(\mathbb{C})$. The element $I_{G}^{\mathfrak{m}}(q, m)$ therefore can be viewed as an element of $\mathcal{P}_{\mathcal{H}(S)}^{\mathfrak{m}, s, \text { gen }}$, invariant under $\pi_{1}^{\text {top }}\left(U_{Q, M}^{\text {gen }} \cap S(\mathbb{C})\right)$. It can therefore be viewed as an element of $\mathcal{P}_{Q, M}^{\mathfrak{m}}$.

For all $(q, m) \in U_{Q, M}^{\text {gen }}$, its period is given by the convergent Feynman integral

$$
\operatorname{per}\left(I_{G}^{\mathfrak{m}}(q, m)\right)=I_{G}(q, m) .
$$

We can also define (for instance in the case $\left.N_{G} \geq\left(h_{G}+1\right) d / 2\right)$ a variant:

$$
I_{G}^{\mathfrak{m}}(q, m)^{\prime}=\left[\operatorname{mot}_{G}^{\prime},\left[\pi_{G}^{*} \omega_{G}(q, m)\right],\left[\sigma_{G}\right]\right]^{\mathfrak{m}} \in \mathcal{P}_{Q, M}^{\mathfrak{m}} .
$$

Since the action of the de Rham Galois group on motivic Feynman integrals will generate new integrands, and since quantum field theory (e.g., gauge theories) naturally produce integrands with numerators, we make the following definition.

Definition 7.3. A motivic Feynman period of type $(Q, M)$ is

$$
I_{G}^{\mathfrak{m}}(\omega)=\left[\operatorname{mot}_{G},[\omega],\left[\sigma_{G}\right]\right]^{\mathfrak{m}} \in \mathcal{P}_{Q, M}^{\mathfrak{m}}
$$

where $[\omega] \in \omega_{d R}^{\text {gen }}\left(\operatorname{mot}_{G}\right)$ is any relative de Rham cohomology class at the generic point. Write

$$
I_{G}(\omega)=\operatorname{per}\left(I_{G}^{\mathfrak{m}}(\omega)\right)
$$

which we call a Feynman period. It is a multivalued meromorphic function on $K_{Q, M}^{\text {gen }}(\mathbb{C})$. Note that graphs for which the Feynman amplitude $\omega_{G}(q, m)$ does not converge may still have non-trivial Feynman periods. The notion of Feynman period is therefore more general, but contains, the notion of Feynman amplitude. Feynman periods may not necessarily always have a physical interpretation.

One can consider a variant for $N_{G} \geq(h+1) d / 2$, by replacing $\operatorname{mot}_{G}$ by $\operatorname{mot}_{G}^{\prime}$.

Remark 7.4. Many, but not all, cohomology classes in $\omega_{d R}^{\mathrm{gen}}\left(\operatorname{mot}_{G}\right)$ are of the form $\left[\pi_{G}^{*}(\omega(q, m))\right]$, where $\omega(q, m)$ is a Feynman integrand with numerator $(6.13)$. 
7.3. Coaction formula and cosmic Galois group. By [17] §8.2, the rings of periods considered above admit a coaction

$$
\Delta: \mathcal{P}_{Q, M}^{\mathfrak{m}} \longrightarrow \mathcal{P}_{Q, M}^{\mathfrak{m}} \otimes_{k_{Q, M}} \mathcal{P}_{Q, M}^{\mathfrak{d r}} .
$$

Applied to a motivic Feynman period we have the formula [17] (2.3)

$$
\Delta I_{G}^{\mathfrak{m}}(\omega)=\sum_{e_{i}}\left[\operatorname{mot}_{G},\left[\sigma_{G}\right], e_{i}\right]^{\mathfrak{m}} \otimes\left[\operatorname{mot}_{G}, e_{i}^{\vee},[\omega]\right]^{\mathfrak{d r}}
$$

and the corresponding variant in which $I$ and $\operatorname{mot}_{G}$ are replaced by $I^{\prime}$ and $\operatorname{mot}_{G}^{\prime}$. In this formula, $e_{i}$ is a basis of $\omega_{d R}^{\mathrm{gen}}\left(\operatorname{mot}_{G}\right)$ and $e_{i}^{\vee}$ the dual basis. The elements on the right-hand side of the tensor product in (7.2) are again motivic Feynman periods of $G$.

It is customary in the physics literature to restrict to one-particle irreducible graphs. Likewise, we shall restrict to the larger class of motic graphs.

Definition 7.5. The comodule of de Rham Feynman periods of type $(Q, M)$ is the subspace $\mathcal{F} \mathcal{P}_{Q, M}^{\mathfrak{d r}} \subset \mathcal{P}_{Q, M}^{\mathfrak{d r}}$ spanned by de Rham Feynman periods

$$
\left[\operatorname{mot}_{G}, f, v\right]^{\mathfrak{d r}}, \quad \text { where } v \in \omega_{d R}^{\mathrm{gen}}\left(\operatorname{mot}_{G}\right) \text { and } f \in \omega_{d R}^{\mathrm{gen}}\left(\operatorname{mot}_{G}\right)^{\vee},
$$

and $G$ is motic of type $(Q, M)$. The coproduct is given by the formula

$$
\Delta\left[\operatorname{mot}_{G}, f, v\right]=\sum_{e_{i}}\left[\operatorname{mot}_{G}, f, e_{i}\right]^{\mathfrak{o r}} \otimes\left[\operatorname{mot}_{G}, e_{i}^{\vee}, v\right]^{\mathfrak{d r}},
$$

where $e_{i}$ is a basis of $\omega_{d R}^{\text {gen }}\left(\operatorname{mot}_{G}\right)$ and $e_{i}^{\vee}$ the dual basis as above. It is not an algebra since it is not closed under products when $(Q, M) \neq(0,0)$.

The space of motivic Feynman periods of type $(Q, M)$ is the subspace

$$
\mathcal{F P}_{Q, M}^{\mathfrak{m}} \subset \mathcal{P}_{Q, M}^{\mathfrak{m}}
$$

spanned by motivic Feynman periods $I_{G}^{\mathfrak{m}}(\omega)$, where $G$ is motic of type $(Q, M)$. It is a right comodule over $\mathcal{F P}_{Q, M}^{\mathrm{dr}}$. The coaction is given by equation (7.2):

$$
\Delta: \mathcal{F P}_{Q, M}^{\mathfrak{m}} \longrightarrow \mathcal{F} \mathcal{P}_{Q, M}^{\mathfrak{m}} \otimes_{k_{Q, M}} \mathcal{F} \mathcal{P}_{Q, M}^{\text {dr }}
$$

Define the cosmic Galois group $C_{Q, M}$ for convergent Feynman periods to be

$$
C_{Q, M}=\operatorname{Spec} \mathcal{F} \mathcal{P}_{Q, M}^{\mathfrak{d r}} .
$$

It is an affine group scheme over $k_{Q, M}$, and acts on $\omega_{d R}^{\text {gen }}\left(\operatorname{mot}_{G}\right)$ for all $G$ motic of type $(Q, M)$. The coaction (7.3) is equivalent to a group action

$$
C_{Q, M} \times \mathcal{F P}_{Q, M}^{\bullet} \longrightarrow \mathcal{F P}_{Q, M}^{\bullet} \quad \text { with } \bullet=\mathfrak{m}, \mathfrak{d r} .
$$

The cosmic Galois group itself should be taken with a pinch of salt (in the same way as the Galois group of all algebraic numbers): it is an enormous pro-algebraic group scheme over $k_{Q, M}$, and in practice one is interested in its finite-dimensional representations. 
7.4. First applications. The notion of motivic Feynman amplitude leads to a number of immediate consequences. We briefly mention just a few.

Definition 7.6. The representation associated to a motivic Feynman period $I_{G}^{\mathfrak{m}}(\omega)$ is the representation of $C_{Q, M}$ which it generates:

$$
V_{G}(\omega) \subset \mathcal{F P}_{Q, M}^{\mathfrak{m}}
$$

The Galois conjugates of a motivic Feynman period are the elements of $V_{G}(\omega)$, or equivalently, the elements of the comodule it generates under the coaction (7.2).

(1) (Weight filtration). We can speak of the weights of Feynman periods. Say that a motivic Feynman period $I_{G}^{\mathfrak{m}}(\omega)$ is of weight at most $n$ if

$$
I_{G}^{\mathfrak{m}}(\omega) \in W_{n} \mathcal{F P}_{Q, M}^{\mathfrak{m}}
$$

This holds in particular if $\omega \in W_{n} \omega_{d R}^{\mathrm{gen}}\left(\operatorname{mot}_{G}\right)$. Note that the weight is a filtration, not a grading, except in very special circumstances. We can say that a Feynman period is of weight $\leq n$ if it is the period per $I_{G}^{\mathfrak{m}}(\omega)$ of a motivic Feynman period $I_{G}^{\mathfrak{m}}(\omega)$ of weight $\leq n .{ }^{13}$

(2) (Picard-Fuchs equations). There is an integrable connection

$$
\nabla: \mathcal{F} \mathcal{P}_{Q, M}^{\mathfrak{m}} \longrightarrow \mathcal{F} \mathcal{P}_{Q, M}^{\mathfrak{m}} \otimes_{k_{Q, M}} \Omega_{k_{Q, M}}^{1}
$$

See [17] $\S 7.4$ for its compatibilities with the period homomorphism and coaction. It follows from general theory and the construction of $\operatorname{mot}_{G}$ that Feynman periods are solutions to differential equations of Fuchsian type.

(3) (Invariants attached to $V_{G}(\omega)$ ). The representation $V_{G}(\omega)$ of a Feynman period carries a barrage of new information. This is discussed in [17]. For example, a motivic Feynman period has a rank (the dimension of $V_{G}(\omega)$ ); a Hodge polynomial; and a filtration by the unipotency degree (this is the coradical filtration on $\mathcal{F P}_{Q, M}^{\mathfrak{m}}$ induced by the coaction (7.3).).

(4) (Mixed Tate amplitudes). Call a motivic Feynman period mixed Artin-Tate if it is equivalent to a motivic period of a variation of Hodge-Tate type (all Hodge numbers $h_{p, q}=0$ unless $p=q$ ). Call it mixed Tate if it is equivalent to a motivic period of $H$ where $\operatorname{gr}_{n}^{W} H$ is zero if $n$ is odd, and a direct sum of constant Tate objects $\mathbb{Q}(-k)$ if $k=2 n$ is even. The weight filtration is a grading on such motivic periods. This case is discussed in $\S 7.7$.

Remark 7.7. The Betti realisation of $\operatorname{mot}_{G}$ is a local system on the complex points of some open $S=K_{Q, M}^{\mathrm{gen}} \backslash L_{G}$, where $L_{G}$ is a closed subscheme we call the Landau variety $^{14}$ of $G$. The fiber of this local system at a point $s$ carries an action of the fundamental group $\pi_{1}^{\text {top }}\left(K_{Q, M}^{\text {gen }}(\mathbb{C}) \backslash L_{G}(\mathbb{C}), s\right)$, corresponding to monodromy of amplitudes, and commutes with the action of $C_{Q, M}$. In these notes, it plays a subordinate role because we do not know how to control $L_{G}$ in general.

\footnotetext{
${ }^{13}$ The Hodge-theoretic weights here are double those commonly used in physics and the field of multiple zeta values and polylogarithms. For example, $\zeta(3)$ has weight 6 and not 3 . If one wishes to divide the weight by two, one will encounter half-integral weights. The simplest example of this phenomenon occurs already for amplitudes which are given by elliptic integrals.

${ }^{14}$ This is more complex [18] than the Landau variety as commonly understood in the physics literature. The Landau equations in the classical sense describe a certain subset, but by no means all, of the components of $L_{G}$.
} 
7.5. Face relations. The recursive structure of graph motives gives rise to relations between the periods of different Feynman graphs.

Let $G$ be a motic Feynman graph. From theorem 6.5, the irreducible components of the divisor $D \subset P^{G}$ define morphisms 'inclusion of facets':

$$
\begin{array}{rlrll}
i_{e} & : & P^{G / e} \backslash Y_{G / e} & \hookrightarrow & P^{G} \backslash Y_{G} \\
i_{\gamma} & : & \left(P^{\gamma} \backslash Y_{\gamma}\right) \times\left(P^{G / \gamma} \backslash Y_{G / \gamma}\right) & \hookrightarrow & P^{G} \backslash Y_{G}
\end{array}
$$

where $e \in E_{G}$ or $\gamma \subsetneq E_{G}$ is a motic subgraph. They induce morphisms of relative cohomology as described in [17], §10.3.

Theorem 7.8. The maps $i_{e}, i_{\gamma}$ induce morphisms

$$
\begin{aligned}
i_{e}: \operatorname{mot}_{G / e} & \longrightarrow \operatorname{mot}_{G} \\
i_{\gamma}: \operatorname{mot}_{\gamma} \otimes \operatorname{mot}_{G / \gamma} & \longrightarrow \operatorname{mot}_{G}
\end{aligned}
$$

in the category $\mathcal{H}(S)$, where $S$ is a Zariski open subset of $K_{Q, M}^{\text {gen }}$ on which the above objects are defined. On the Betti realisation, this map gives

$$
\omega_{B}\left(i_{e}\right)^{\vee}\left[\sigma_{G}\right]=\left[\sigma_{G / e}\right] \quad \text { and } \quad \omega_{B}\left(i_{\gamma}\right)^{\vee}\left[\sigma_{G}\right]=\left[\sigma_{\gamma}\right] \otimes\left[\sigma_{G / \gamma}\right] .
$$

This implies equalities of motivic periods in $\mathcal{F} \mathcal{P}_{Q, M}^{\mathfrak{m}}$

$$
\begin{aligned}
{\left[\operatorname{mot}_{G / e}, \omega, \sigma_{G / e}\right]^{\mathfrak{m}} } & =\left[\operatorname{mot}_{G}, \omega_{d R}^{\operatorname{gen}}\left(i_{e}\right)(\omega), \sigma_{G}\right]^{\mathfrak{m}} \\
{\left[\operatorname{mot}_{\gamma}, \omega_{1}, \sigma_{\gamma}\right]^{\mathfrak{m}} \times\left[\operatorname{mot}_{G / \gamma}, \omega_{2}, \sigma_{G / \gamma}\right]^{\mathfrak{m}} } & =\left[\operatorname{mot}_{G}, \omega_{d R}^{\operatorname{gen}}\left(i_{\gamma}\right)\left(\omega_{1} \otimes \omega_{2}\right), \sigma_{G}\right]^{\mathfrak{m}} .
\end{aligned}
$$

Proof. Theorem 6.5 implies that the facet map

$$
i_{e}: P^{G / e} \backslash Y_{G / e} \stackrel{\sim}{\rightarrow} D_{e} \backslash\left(Y_{G} \cap D_{e}\right)
$$

is an isomorphism of stratified spaces, where the stratification is induced by the divisor $D_{e} \cap\left(D \backslash D_{e}\right)$ on both sides of the equation. The face morphisms defined in [17] $\$ 10.4$, therefore define the required $\operatorname{morphism}_{\operatorname{mot}_{G / e}} \rightarrow \operatorname{mot}_{G}$ in the category $\mathcal{H}(S)$. In the Betti realisation, this map corresponds to taking the boundary component of a relative homology cycle which is contained in $D_{e}(\mathbb{C})$. By corollary 5.2 this gives exactly $\sigma_{G} \cap D_{e}(\mathbb{C}) \cong \widetilde{\sigma}_{G} \cap D_{e}(\mathbb{C}) \cong \widetilde{\sigma}_{G / e} \cong \sigma_{G / e}$.

The corresponding equation in the case of the face map $i_{\gamma}$ follows from a similar argument, using the isomorphism of stratified spaces

$$
i_{\gamma}:\left(P^{\gamma} \backslash Y_{\gamma}\right) \times\left(P^{G / \gamma} \backslash Y_{G / \gamma}\right) \stackrel{\sim}{\rightarrow} D_{\gamma} \backslash\left(Y_{G} \cap D_{\gamma}\right),
$$

where the stratification on the left is the product of the stratifications induced by the divisors (5.2) on $P^{\gamma}$ and $P^{G / \gamma}$, which follows from theorem 6.5.

This theorem implies an analogous statement corresponding to the inclusions of faces of higher codimension. We shall call (7.7) the face relations. ${ }^{15}$ They are preserved by the action of the cosmic Galois group $C_{Q, M}$.

\footnotetext{
${ }^{15}$ Although the face relations are stated here as identities between periods of a category of Betti and de Rham realisations, it is obvious that they should be true 'motivically' for any reasonable definition of the word, since they come from the morphisms $i_{e}, i_{\gamma}$ of algebraic varieties.
} 
7.6. Multiplicative structure. It is important to observe that in the second equation of (7.7), one of $\gamma$ or $G / \gamma$ has no dependence on external kinematics (4.3), and hence defines a constant motivic period in the sense of [17] §7.3.1. Therefore even if one is only interested in processes with $Q$ external momenta and $M$ non-zero particle masses, one is inexorably led to consider the case of Feynman integrals with no kinematics (these only depend on the graph polynomial $\Psi_{G}$ and not $\left.\Xi_{G}(q, m)\right)$. The following proposition gives another example of the special role played by periods of Feynman graphs of type $(0,0)$.

Proposition 7.9. The vector space $\mathcal{F P}_{Q, M}^{\mathfrak{m}}$ is closed under multiplication by elements of $\mathcal{F P}_{0,0}^{\mathfrak{m}}$. In other words, multiplication defines a map

$$
\mathcal{F P}_{0,0}^{\mathfrak{m}} \otimes_{\mathbb{Q}} \mathcal{F} \mathcal{P}_{Q, M}^{\mathfrak{m}} \longrightarrow \mathcal{F} \mathcal{P}_{Q, M}^{\mathfrak{m}}
$$

Thus the space of Feynman periods of type $(0,0)$ is a commutative algebra, and the space of Feynman periods of type $(Q, M)$ is a module over it.

Proof. Let $\gamma$ be a motic graph of type $(0,0)$, and let $H$ be a motic Feynman graph of type $(Q, M)$. Choose any vertex $v$ of $H$. By inserting $\gamma$ into $v$ and attaching the edges of $H$ (both internal and external) which are incident to $v$ to vertices of $\gamma$ in any way, defines a new graph $G$ of type $(Q, M)$, such that $\gamma \subset G$ is motic and $G / \gamma \cong H$ is also motic. By theorem 3.6 (ii), $G$ is motic. Now apply the face equation in the second line of (7.7). This proves that a product of motivic periods of $\gamma$ and $H$ are motivic periods of $G$.

The same statement is evidently true also for de Rham periods: multiplication yields a map $\mathcal{F P}_{0,0}^{\mathfrak{d r}} \otimes_{\mathbb{Q}} \mathcal{F} \mathcal{P}_{Q, M}^{\mathfrak{d r}} \longrightarrow \mathcal{F P}_{Q, M}^{\mathfrak{d r}}$, and $\mathcal{F} \mathcal{P}_{0,0}^{\mathfrak{d r}}$ is an algebra.

7.7. Single-valued amplitudes and symbols. The right-hand side of the coaction involves de Rham Feynman periods. It is an important problem to determine properties of the right-hand side of the coaction and try to interpret these quantities physically. Note that they do not have periods.

As a substitute, we have a notion of single-valued period [17] §8.3. Restricting to the space of de Rham Feynman amplitudes, it is a homomorphism

$$
\mathbf{s}^{\mathfrak{m}}: \mathcal{F} \mathcal{P}_{Q, M}^{\mathfrak{d r}} \longrightarrow \mathcal{P}_{Q, M}^{\mathfrak{m}} \otimes_{\mathbb{Q}} \overline{\mathcal{P}}_{Q, M}^{\mathfrak{m}} .
$$

Composing with the period homomorphism defines a real analytic single-valued function on $K_{Q, M}^{\text {gen }}(\mathbb{C})$, with possible poles, which we call the single-valued period.

In the case when a de Rham Feynman period $\xi$ is unipotent [17] 99.2 , we can associate various notions of symbol to it. This holds in particular when $\xi$ is mixed Artin-Tate, and therefore can be applied to large classes of Feynman periods. The symbol is a class in the reduced bar construction

$$
\operatorname{smb}(\xi) \in H^{0}\left(\mathbb{B}\left(\Omega_{k_{Q, M}}^{1}\right)\right)
$$

and has an invariant called its length. The cohomological symbol $\mathrm{cmb}_{\ell}(\xi)$ is the length- $\ell$ part of the symbol of length $\leq \ell$ and can be viewed as an integrable word in logarithmic one-forms on $k_{Q, M}$. See [17] $\S 9.4$. This notion of symbol is a generalisation of the notion of symbol used extensively in the physics literature. 
7.8. Total Feynman motive. Using the face maps we can take the limit over all graphs of type $(Q, M)$ and assemble them into a single object.

Definition 7.10. The total (convergent) Feynman motive of type $(Q, M)$ is

$$
\operatorname{mot}_{Q, M}=\underset{G}{\lim _{\longrightarrow}} \operatorname{mot}_{G}
$$

where the limit is over all motic graphs of type $(Q, M)$ and the morphisms are given by the face maps $i_{e}$ of (7.6). It is an ind object of $\operatorname{Rep}\left(C_{Q, M}\right)$.

The periods of (7.8) do not a priori contain the renormalised amplitudes of graphs. It would be interesting to write down the object which does capture the amplitudes of all graphs of type $(Q, M)$ after renormalisation. Note also that (7.8) has a number of variants: for instance one can take the limit over all planar graphs, or indeed any family of graphs closed under the operation of contracting edges.

\section{WEIGHTS AND STABILITY}

We now apply results on weight filtrations from [17] 99.

8.1. Motives of descendants. We attach a motive to the motic descendants of graphs (defined in $\S 4.2$ ), in the following way. To a motic descendent $\gamma_{1} \otimes \ldots \otimes \gamma_{n}$ of $G$ of type $(Q, M)$, assign the object

$$
\operatorname{mot}\left(\gamma_{1} \otimes \ldots \otimes \gamma_{n}\right)=\operatorname{mot}\left(\gamma_{1}\right) \otimes \ldots \otimes \operatorname{mot}\left(\gamma_{n}\right) \quad \in \quad \mathcal{H}(S),
$$

where $S$ is some Zariski-open in the space of kinematics $K_{Q, M}^{\mathrm{gen}}$. Define a Betti class in $\left(\omega_{B} \operatorname{mot}\left(\gamma_{1} \otimes \ldots \otimes \gamma_{n}\right)\right)^{\vee}$ by

$$
\left[\sigma_{\gamma_{1} \otimes \ldots \otimes \gamma_{n}}\right]=\left[\sigma_{\gamma_{1}}\right] \times \ldots \times\left[\sigma_{\gamma_{n}}\right] .
$$

Exactly one of the graphs $\gamma_{i}$ is of type $(Q, M)$; the others are of type $(0,0)$. There is a canonical face map $\operatorname{mot}\left(\gamma_{1}\right) \otimes \ldots \otimes \operatorname{mot}\left(\gamma_{n}\right) \rightarrow \operatorname{mot}(G)$ that sends $\sigma_{\gamma_{1} \otimes \ldots \otimes \gamma_{n}}$ to $\sigma_{G}$, defined by inclusion of the corresponding face of $\sigma_{G}$, or by iterating (7.6). By the face equations (7.7) the motivic periods of descendants of $G$ (i.e., the motivic periods $\left.\left[\operatorname{mot}\left(\gamma_{1} \otimes \ldots \otimes \gamma_{n}\right),\left[\sigma_{\gamma_{1} \otimes \ldots \otimes \gamma_{n}}\right],[\omega]\right]^{\mathfrak{m}}\right)$ are also motivic periods of $G$.

The degree (4.5) coincides with the cohomological degree of the corresponding motive, and the dimension of the corresponding facet in the Feynman polytope.

Lemma 8.1. Let $G$ be a Feynman graph of type $(Q, M)$. Then $W_{0} \operatorname{mot}(G)=\mathbb{Q}(0)$.

Proof. Let $N_{G}=\left|E_{G}\right| \geq 2$. Apply the results from [17], corollary 10.6. The irreducible components of $D \subset P^{G}$, defined in (5.2), are in one-to-one correspondence with the facets of the Feynman polytope $\widetilde{\sigma}_{G}$, whose boundary is homotopic to an $n$-1-sphere, where $n=N_{G}-1$. Its cohomology in degree $n-1$ is one-dimensional. The case when $N_{G} \leq 1$ is trivial, since $P^{G}$ is a just a point.

Remark 8.2. If $\operatorname{deg}(G)=0$ then $\operatorname{mot}(G)=H^{0}(\operatorname{Spec} \mathbb{Q})=\mathbb{Q}(0)$. It follows that there are only finitely many motives attached to the set of all possible tensors $\gamma_{1} \otimes \ldots \otimes \gamma_{n}$ of bounded degree. 
8.2. Weight relations. Let $G$ be a Feynman graph of type $(Q, M)$. Recall that $\operatorname{mot}(G)=H^{N_{G}-1}\left(P^{G} \backslash Y_{G}, D \backslash\left(D \cap Y_{G}\right)\right)$. Let $D^{(k)}$ denote the union of the $k$ dimensional facets of $D$. Then there is a morphism

$$
W_{k} H^{k}\left(D^{(k)} \backslash D^{(k)} \cap Y_{G}\right) \longrightarrow W_{k} \operatorname{mot}(G)
$$

which is surjective (proposition 10.4 in [17]). The dual map on Betti homology sends the class of the Feynman polytope $\left[\sigma_{G}\right]$ to the class of the union $\left[\sigma_{G}^{(k)}\right]$ of its $k$-dimensional facets. This implies the following theorem.

Theorem 8.3. Every motivic period of $\operatorname{mot}(G)$ of weight $\leq k$ is equivalent to a motivic period of the form $\left[H^{k}\left(D^{(k)} \backslash D^{(k)} \cap Y_{G}\right),\left[\sigma_{G}^{(k)}\right],[\omega]\right]^{\mathfrak{m}}$.

Its period is a $k$-dimensional integral

$$
\int_{\sigma_{G}^{(k)}} \omega
$$

where $[\omega] \in H_{d R}^{k}\left(D^{(k)} \backslash D^{(k)} \cap Y_{G}\right)$. By triangulating the domain of integration into affine regions, as discussed in Appendix 1, and taking limits, this integral can be written as a sum of regularised limits of integrals over each facet of $D^{(k)}$. Since each facet is isomorphic to a product of graph hypersurface complements of motic descendants of $G$ of total degree $\leq k$, this gives a heuristic justification, via the argument of $\S 11.4$, for conjecture 1 . The missing ingredient is to define a notion of regularisation on the level of motivic periods, which is beyond the scope of the notes. This would also have applications to the theory of renormalisation.

Remark 8.4. It is not the case that $P^{G} \backslash Y_{G}$ are affine, since there exist cohomology classes in $P^{G} \backslash Y_{G}$ of degree greater than its dimension. Therefore proposition 10.7 in [17] cannot be applied, and we cannot deduce that the face maps surject onto $W_{k} \operatorname{mot}_{G}$, which would have implied that all motivic periods of $\operatorname{mot}_{G}$ of weight $\leq k$ are in the image of the face maps. We do not know, therefore, whether the motivic periods of weight $\leq k$ of $\operatorname{mot}(G)$ relative to $\sigma_{G}$ are generated by the motivic periods of weight $\leq k$ of its motic descendents of degree $\leq k$, as one might hope.

8.3. Stability. A first application of this theory is to show that the weight-graded parts of the total motive $\operatorname{gr}_{k}^{W} \operatorname{mot}_{Q, M}$ stabilize.

Theorem 8.5. Let $G$ be a Feynman graph of type $(Q, M)$. Then $\operatorname{gr}_{k}^{W} \operatorname{mot}(G)$ is a sub-quotient of

$$
\bigoplus_{\gamma=\gamma_{1} \times \ldots \times \gamma_{r}} \bigoplus_{i_{1}+\ldots+i_{r} \leq k} \operatorname{gr}_{k}^{W} H^{i_{1}}\left(P^{\gamma_{1}} \backslash Y_{\gamma_{1}}\right) \otimes \ldots \otimes H^{i_{r}}\left(P^{\gamma_{r}} \backslash Y_{\gamma_{r}}\right)
$$

where the direct sum is over motic descendents of $G$ of degree $i_{1}+\ldots+i_{r} \leq k$.

Proof. Apply corollary of 10.5 of [17] to $\operatorname{mot}(G)$. We deduce that $\operatorname{gr}_{k}^{W} \operatorname{mot}(G)$ is a sub-quotient of $\bigoplus_{|I| \geq n-k} \operatorname{gr}_{k}^{W} H^{n-|I|}\left(D_{I} \backslash D_{I} \cap Y_{G}\right)_{/ S}$, where $D_{I}$ are the codimension $|I|$, and hence dimension $n-|I| \leq k$ facets of $D$. By theorem 6.5,

$$
D_{I} \backslash\left(D_{I} \cap Y_{G}\right) \cong P^{\gamma_{1}} \backslash Y_{\gamma_{1}} \times \ldots \times P^{\gamma_{r}} \backslash Y_{\gamma_{r}},
$$

where $\gamma_{1} \otimes \ldots \otimes \gamma_{r}$ is a descendant of $G$ of degree $n-|I|$. The statement follows from the Kunneth formula. (Note that the degree of $\gamma_{i_{j}}$ is unrelated to $i_{j}$.) 
The theorem gives a constraint on the Hodge polynomials of the motivic periods of $G$ of weight $\leq k$. Combined with the Galois coaction, this gives a constraint on motivic periods to all orders. For example, we can deduce the following corollary.

Corollary 8.6. Suppose that all $1 P I$ graphs of type $(0,0)$ up to $N+1$ edges have mixed Tate cohomology in all degrees. Then the Galois conjugates of any motivic Feynman amplitude of type $(0,0)$ which is of weight $\leq N$ is mixed Tate.

By computation, one knows that the assumption of the corollary is true for $\mathrm{N}$ up to about 10. This corollary therefore already provides a very strong constraint on the possible periods which can occur to all orders in perturbation theory.

We can be more precise and try to bound not only the weight-graded parts, but also the extensions between them. This is the spirit of conjecture 1 . In this direction we can prove the following weaker version of the conjecture.

Theorem 8.7. The space $W_{k} \mathcal{F} \mathcal{P}_{Q, M}^{\mathfrak{m}}$ is finite-dimensional.

Remark 8.8. A proof of this theorem is given in $\S 8.5$ using affine models. A different way to find an upper bound for the vector space of periods per $W_{k} \mathcal{F P}_{Q, M}^{\mathfrak{m}}$ is as follows. Apply theorem 8.3 to write any motivic period of weight $\leq k$ as a motivic period of a union $D^{(k)}$ of $k$-dimensional facets. Using our canonical affine covering of $\mathbb{P}^{G}$, and triangulating as in Appendix 1, its period can be written as a sum of periods of affine pieces of each facet. Since the number of graphs with at most $k+1$ edges is finite, and each facet is a product of such graphs, there are only finitely many such affine pieces. This argument gives a crude but effective upper bound for the periods of weight $\leq k$ in terms of periods of the relative cohomology of (blowups of ) graph hypersurfaces of graphs with $\leq k+1$ edges where we now integrate over a cube $[0,1]^{n}$ (see comments after corollary 11.2). These can in principle be computed for small $k$.

8.4. A principle of small graphs. Combined with the action of the cosmic Galois group, $C_{Q, M} \times \mathcal{F P}_{Q, M}^{\mathfrak{m}} \longrightarrow \mathcal{F P}_{Q, M}^{\mathfrak{m}}$ or rather, the fact that the motivic periods of any graph $G$ are closed under the action of $C_{Q, M}$, theorem 8.7 gives constraints on Feynman amplitudes to all orders. The space $W_{k} \mathcal{F} \mathcal{P}_{Q, M}^{\mathfrak{m}}$ is determined from the finitely many 'small' graphs with at most $k+1$ edges. This forces constraints on the Galois conjugates of Feynman periods of all graphs. For an example, see $§ 9.3$.

8.5. Affine motive and proof of finiteness theorem 8.7. Let $G$ be a Feynman graph of type $(Q, M)$. Define the affine motive $\operatorname{mot}_{G}^{a}$ of $G$ in an identical way to definition 6.4 except that we replace $P^{G}$ with its affine open $A^{G}$ of $\S 5.4$ :

$$
\operatorname{mot}_{G}^{a}=H^{N_{G}-1}\left(A^{G} \backslash Y_{G}, D \backslash\left(D \cap Y_{G}\right)\right)_{/ S},
$$

as an object of $\mathcal{H}(S)$, for some Zariski-open $S$ in the space of kinematics $K_{Q, M}^{\text {gen }}$. It follows from the construction of $A^{G}$, which is obtained by removing from $P^{G}$ hyperplanes with strictly positive coefficients, that

$$
\tilde{\sigma}_{G} \subset A^{G}(\mathbb{C}) \text {. }
$$

See the proof of theorem 6.7. Thus $\operatorname{mot}_{G}^{a}$ has a canonical Betti element defined by $\sigma_{G}$ in an identical manner to definition 6.8:

$$
\left.\left[\sigma_{G}\right] \in \Gamma\left(U_{Q, M}^{\text {gen }},\left(\operatorname{mot}_{G}^{a}\right)_{B}^{\vee}\right)\right)
$$


Furthermore, the inclusion $A^{G} \backslash Y_{G} \subset P^{G} \backslash Y_{G}$ defines a morphism of objects

$$
i: \operatorname{mot}_{G} \longrightarrow \operatorname{mot}_{G}^{a}
$$

in $\mathcal{H}(S)$, which respects the Betti-clases $\left[\sigma_{G}\right]$ on both sides. This morphism defines an equivalence on the level of motivic periods [17] 2 .

Corollary 8.9. There is an equality of motivic periods

$$
I_{G}^{\mathfrak{m}}(\omega)=\left[\operatorname{mot}_{G}^{a}, i_{d R}[\omega],\left[\sigma_{G}\right]\right]^{\mathfrak{m}},
$$

where the left-hand side was defined in definition 7.3. In particular, every Feynman period of $G$ is a period of the affine motive $\operatorname{mot}_{G}^{a}$. We could thus use the affine motives $\operatorname{mot}_{G}^{a}$ to study the weights, representations and so on of Feynman periods.

Note that the affine motive is excessively large: it has many periods which are unrelated to Feynman graphs. One advantage of the previous corollary, however, is that it enables us to express every Feynman period as an integral of a globallydefined algebraic differential form, since, by a theorem due to Grothendieck, the de Rham cohomology of an affine variety is the cohomology of the complex of global regular differential forms. The price to pay is that the integrand may involve linear denominators of the form $\sum_{e \in \gamma} \alpha_{e}$, where $\gamma$ is a motic subgraph of $G$. This remark may or may not be of practical use in computing Feynman periods.

Definition 8.10. Let us call the affine motivic periods of a graph $G$ to be the space of motivic periods of $\operatorname{mot}_{G}^{a}$ with respect to $\sigma_{G}$. By the above remarks, it contains the space of motivic periods of $G$.

Now, it follows from the product structure on the spaces $A^{G}$ that the analogue of theorem 6.5 holds on replacing $P^{G}$ by $A^{G}$, and hence the face relations (theorem 7.8) hold for $\operatorname{mot}_{G}^{a}$. Now apply proposition 10.7 in [17], which exploits Artin vanishing for the cohomology of affine schemes, to deduce the following theorem.

Theorem 8.11. The affine motivic periods of $G$ of weight $\leq k$ are $k_{Q, M-l i n e a r}$ combinations of the affine motivic periods of motic descendents of $G$ of degree $\leq k$.

Since there are only finitely many descendents of bounded degree (remark 8.2), we immediately deduce theorem 8.7.

\section{The constant cosmic Galois group}

The motivic periods of graphs of type $(Q, M)=(0,0)$, which have no dependence on external kinematics, play a special role in the Galois theory of all Feynman amplitudes and are of particular number-theoretic interest.

9.1. A Galois theory of graph periods. We recall the main definitions in this case. We shall make no restrictions on the 'physicality' of the graphs under consideration, i.e., our graphs can have arbitrary vertex-degrees for the time being.

Definition 9.1. For any graph $G$ of type $(0,0)$, recall that

$$
\operatorname{mot}(G)=H^{N_{G}-1}\left(P^{G} \backslash Y_{G}, D \backslash\left(D \cap Y_{G}\right)\right),
$$

is an effective object in the category $\mathcal{H}$ defined in [17] $\S 2$. It consists of triples $\left(V_{B}, V_{d R}, c\right)$ where $V_{B}, V_{d R}$ are finite-dimensional $\mathbb{Q}$ vector spaces, and $c$ is an isomorphism $c: V_{d R} \otimes \mathbb{C} \stackrel{\sim}{\rightarrow} V_{B} \otimes \mathbb{C}$. These spaces are equipped with filtrations which define a mixed Hodge structure. In this context, the word effective means that the 
Hodge numbers of $\operatorname{mot}(G)$ satisfy $h_{p, q}=0$ if $p$ or $q$ is negative. Denote the ring of motivic graph periods to be the $\mathbb{Q}$-vector space spanned by the motivic periods:

$$
\mathcal{F} \mathcal{P}_{0,0}^{\mathfrak{m}}=\left\langle\left[\operatorname{mot}(G),\left[\sigma_{G}\right],[\omega]\right]^{\mathfrak{m}}\right\rangle_{\mathbb{Q}} \quad \subset \quad \mathcal{P}_{\mathcal{H}}^{\mathfrak{m},+}
$$

where $G$ is motic (i.e., $1 \mathrm{PI}$ ) and $\left[\sigma_{G}\right] \in \operatorname{mot}(G)_{B}^{\vee}$ is the canonical Betti framing. It actually lands in the subspace $\mathcal{P}^{\mathfrak{m},+} \subset \mathcal{P}_{\mathcal{H}}^{\mathfrak{m},+}$ defined in [17], Definition 3.4. Define the space of motivic periods of a fixed graph $G$ to be the vector space

$$
\mathcal{F P}^{\mathfrak{m}}(G)=\left\langle\left[\operatorname{mot}(G),\left[\sigma_{G}\right],[\omega]\right]^{\mathfrak{m}} \quad \text { for } \quad[\omega] \in \operatorname{mot}(G)_{d R}\right\rangle_{\mathbb{Q}}
$$

spanned by its motivic graph periods. We say that a graph $G$ has weight at most $n$ and write $w(G) \leq n$ if $W_{n} \mathcal{F P}^{\mathfrak{m}}(G)=\mathcal{F P}^{\mathfrak{m}}(G)$, in accordance with $\S 7.4 .{ }^{16}$

In the special case when $G$ is overall log-divergent $\left(N_{G}=2 h_{G}\right)$ and primitive $\left(N_{\gamma}>2 h_{\gamma}\right.$ for all $\left.\gamma \subsetneq E_{G}\right)$, the mixed Hodge structure underlying $\operatorname{mot}(G)$ coincides with the graph motive of [10]. In this case the amplitude is given by the integral

$$
I_{G}=\operatorname{per}\left(\left[\operatorname{mot}(G),\left[\omega_{G}\right],\left[\sigma_{G}\right]\right]^{\mathfrak{m}}\right)=\int_{\sigma_{G}} \frac{\Omega_{G}}{\Psi_{G}^{2}}
$$

which converges by lemma 6.10 . Thanks to $[13,48]$ we know hundreds of examples of periods (9.2), and many identities between them. These identities, when taken alone, do not give much control on the possible integrals (9.2), since every new algebra generator is arbitrary. However, if these identities hold on the level of the motivic periods $I_{G}^{\mathfrak{m}}$, as we expect, then when combined with the action of the cosmic Galois group and stability, we obtain a very rigid structure, since the Galois conjugates are constrained by the periods of smaller graphs.

9.2. Invariants and classification. Motivic periods over $\mathbb{Q}$ are studied in some detail in [17], §3. Two constructions worth mentioning are the unipotency degree (or coradical filtration) $C_{i} \mathcal{P}_{\mathcal{H}}^{\mathfrak{m},+}$ and the decomposition into primitives. Say that a motivic Feynman period is of unipotency degree at most $n$ if it lies in $C_{n} \mathcal{F} \mathcal{P}_{0,0}^{\mathfrak{m}}$. In the case of motivic multiple zeta values, the unipotency degree is bounded above by the depth. The decomposition into primitives is a homomorphism [17], 55

$$
\Phi: \operatorname{gr}^{C} \mathcal{F} \mathcal{P}_{0,0}^{\mathfrak{m}} \longrightarrow \operatorname{gr}_{0}^{C} \mathcal{F} \mathcal{P}_{0,0}^{\mathfrak{m}} \otimes_{\mathbb{Q}} T^{c}\left(\operatorname{gr}_{1}^{C} \mathcal{O}\left(U_{\mathcal{H}}^{d R}\right)\right)
$$

where $T^{c}$ denotes the tensor coalgebra, or shuffle algebra, and $\operatorname{gr}_{1}^{C} \mathcal{O}\left(U_{\mathcal{H}}^{d R}\right)$ is a vector space which can be made explicit. The unipotency grading on the left-hand side coincides with the length grading of tensors on the right. This map generalises the (highest-length part of) the decomposition of motivic multiple zeta values into an alphabet of letters $f_{2 n+1}$ to all motivic periods.

We have constructed a map from graphs to representations

$$
\begin{aligned}
\{\text { Graphs of type }(0,0)\} & \longrightarrow \operatorname{Rep}_{\mathbb{Q}}\left(C_{0,0}\right) \\
G & \mapsto \mathcal{F P}^{\mathfrak{m}}(G)
\end{aligned}
$$

where $C_{0,0}$ is the constant cosmic Galois group. This is more subtle than the naive map which sends $G$ to the object $\operatorname{mot}(G)_{d R}$, since it takes into account the Betti framing $\sigma_{G}$. In the notation of [17] $§ 2.4$ we have

$$
\mathcal{F P}^{\mathfrak{m}}(G) \cong\left({ }_{\sigma} \operatorname{mot}(G)\right)_{d R}
$$

\footnotetext{
${ }^{16}$ This is a more subtle notion than the naive weight of a graph defined by the weight of the object $\operatorname{mot}_{G}$ in $\mathcal{H}$. If $W_{n} \operatorname{mot}(G)=\operatorname{mot}(G)$ then it is certainly true that the periods of $G$ have weight $\leq n$, but the examples given below show that the converse is false.
} 
where ${ }_{\sigma} \operatorname{mot}(G)$ denotes the smallest quotient of $\operatorname{mot}(G)$ in the category $\mathcal{H}$ such that $\sigma_{G} \in\left({ }_{\sigma} \operatorname{mot}(G)\right)_{B}^{\vee}$. We can apply any of the invariants of motivic periods defined in [17] to $\mathcal{F P}^{\mathfrak{m}}(G)$. The challenge, then, is to relate invariants of motivic graph periods to topological invariants of their graphs, and find relations between graphs through which the map (9.3) factorizes (see $\$ 9.4$ ).

For example, we called $G$ mixed Artin-Tate if all elements of $\mathcal{F P}^{\mathfrak{m}}(G)$ have Hodge numbers $h_{p, q}=0$ if $p \neq q$. Graphs of vertex-width $\leq 3$ are of this type [18].

Furthermore, let us call $G$ separated ${ }^{17}$ if it satisfies

$$
\left(\operatorname{mot}_{G}\right)_{d R}=W_{0}\left(\operatorname{mot}_{G}\right)_{d R} \oplus F^{1}\left(\operatorname{mot}_{G}\right)_{d R} .
$$

In this case, [17] $\S 4.3$ provides a canonical projection

$$
\pi^{\mathfrak{d} \mathfrak{r}, \mathfrak{m}+}: \mathcal{F}^{\mathfrak{m}}(G) \longrightarrow \mathcal{F} \mathcal{P}^{\mathfrak{d} \mathfrak{r}}(G)
$$

from the motivic periods of $G$ to the de Rham periods of $G$, and gives a handle on the right-hand terms in the motivic coaction. There is evidence to suggest, using methods from [20], that a large class of graphs indeed satisfy this property. In particular, all graphs of mixed Artin-Tate type are separated.

For any separated Feynman graph $G$ of type $(0,0)$, apply the projection followed by the single-valued map $\mathbf{s}^{\mathfrak{m}}[17], \S 4.1$ to obtain a linear map

$$
\mathcal{F} \mathcal{P}^{\mathfrak{m}}(G) \stackrel{\pi^{\mathfrak{d} r, \mathfrak{m}}+}{\longrightarrow} \mathcal{F} \mathcal{P}^{\mathfrak{d r}}(G) \stackrel{\mathbf{s}^{\mathfrak{m}}}{\longrightarrow} \mathcal{P}_{\mathcal{H}}^{\mathfrak{m}}
$$

This defines canonical single-valued versions of its motivic periods $\mathbf{s}^{\mathfrak{m}} \pi^{\mathfrak{d} \mathfrak{r}, \mathfrak{m}+} I_{G}^{\mathfrak{m}}(\omega)$. For multiple zeta values, the corresponding single-valued versions occur in string perturbation theory, and have a physical significance since they relate open and closed superstring amplitudes [53, 55, 33]. Indeed, the formula for the closed string vertex operator for the emission of a closed string state as a product of open string vertex operators precisely mimics the definition of the single-valued motivic periods ([17], last line of $\S 4.1$.

9.3. Small-graphs principle. By way of illustration, we compute the motivic periods of graphs with at most three edges and deduce some non-trivial consequences. We first dispense with two degenerate families of graphs.

Lemma 9.2. If $G$ has a single vertex, or a single loop, then $\operatorname{mot}(G) \cong \mathbb{Q}(0)$.

Proof. Let $n=E_{G}-1$. Suppose that $h_{G}=1$. Then $\Psi_{G}=\sum_{e \in E_{G}} \alpha_{e}$ and $X_{G}$ is a hyperplane $H$. Since $G$ has no non-trivial motic subgraphs, $P^{G}=\mathbb{P}^{n}$ and $P^{G} \backslash Y_{G}=\mathbb{P}^{n} \backslash H \cong \mathbb{A}^{n}$. Similarly, every stratum $D_{I} \cap\left(D_{I} \backslash Y_{G}\right)$ is an affine space and has the cohomology of a point. Therefore the relative cohomology spectral sequence $E_{1}^{p, q}=\bigoplus_{|I|=p} H^{q}\left(D_{I} \cap\left(D_{I} \backslash Y_{G}\right)\right)$, which converges to $\operatorname{mot}(G)$, satisfies $E_{1}^{p, q}=0$ if $q>0$ and hence $\operatorname{mot}(G) \cong \mathbb{Q}(0)$.

Now suppose that $G$ has one vertex. Every subgraph of $G$ is motic, and the graph polynomial is $\Psi_{G}=\prod_{e \in E_{G}} \alpha_{e}$. Since the graph hypersurface $X_{G}$ is the union $L$ of coordinate axes, its strict transform $Y_{G}$ in $P^{G}$ is empty. Therefore

$$
\operatorname{mot}(G)=H^{n}\left(P^{G}, D\right) \cong H^{n}\left(P^{G} \backslash D\right)^{\vee}(-n),
$$

by Poincaré-Verdier duality. Since $P^{G} \backslash D \cong \mathbb{P}^{n} \backslash L \cong \mathbb{G}_{m}^{n}$, the right-hand side is $H^{n}\left(\mathbb{G}_{m}^{n}\right)^{\vee}(-n)=\mathbb{Q}(-1)^{\otimes(-n)}(-n)=\mathbb{Q}(0)$.

\footnotetext{
${ }^{17}$ Or, better, the weaker condition $\sigma\left(\operatorname{mot}_{G}\right)_{d R}=W_{0}\left(\sigma\left(\operatorname{mot}_{G}\right)\right)_{d R} \oplus F^{1}\left(\sigma\left(\operatorname{mot}_{G}\right)\right)_{d R}$
} 
All two-edge motic (i.e. 1PI) graphs are covered by the previous lemma. The four 1PI graphs with three edges are depicted below. Their graph polynomials are underneath. The two outer graphs are trivial by the previous lemma. The

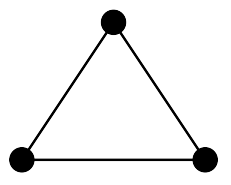

$\alpha_{1}+\alpha_{2}+\alpha_{3}$

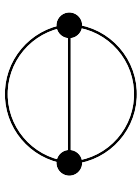

$\alpha_{1} \alpha_{2}+\alpha_{1} \alpha_{3}+\alpha_{2} \alpha_{3}$

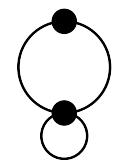

$\alpha_{1}\left(\alpha_{2}+\alpha_{3}\right)$

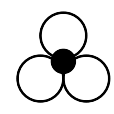

$\alpha_{1} \alpha_{2} \alpha_{3}$

middle two have non-global periods which are in fact trivial (they $\operatorname{satisfy} \operatorname{mot}(G) \cong$ $\mathbb{Q}(0) \oplus \mathbb{Q}(-1))$. This can be seen in two ways: either by a similar analysis to $\S 12$, or by observing that $\operatorname{mot}(G)$ defines a mixed Tate motive which is unramified at all primes. Every Kummer extension of mixed Tate motives which is defined over $\mathbb{Z}$ splits as a direct sum of Tate motives, so $\operatorname{mot}(G)$ has no non-trivial motivic periods.

Lemma 9.3. We have $W_{2} \mathcal{F} \mathcal{P}_{0,0}^{\mathfrak{m}}=W_{0} \mathcal{F} \mathcal{P}_{0,0}^{\mathfrak{m}} \cong \mathbb{Q}$

Proof. By theorem 8.5 and the above calculations, $\operatorname{gr}_{i}^{W} \mathcal{F} \mathcal{P}_{0,0}^{\mathfrak{m}}$ vanishes for $i=1$, and is a direct sum of Tate motives $\mathbb{Q}(0)$ and $\mathbb{Q}(-1)$ for $i=0,2$ respectively since it is generated by the cohomology of graphs of degree $\leq 2$. Therefore $W_{2} \mathcal{F} \mathcal{P}_{0,0}^{\mathfrak{m}}$ is a linear combination of Kummer motivic periods (motivic logarithms), which are the motivic periods of extensions of $\mathbb{Q}(-1)$ by $\mathbb{Q}(0)$. By theorem 8.3 the motivic periods of $\operatorname{mot}(G)$ of weight $\leq 2$ are equivalent to the motivic periods of its 2 -dimensional skeleton $D^{(2)}$, each of whose faces is of one of the above types, or, more trivially, corresponds to a product of two 2-edge graphs (when $\left|E_{G}\right|=2, \mathbb{P}^{G} \backslash Y_{G}$ is either $\mathbb{P}^{1}$ if $G$ has one vertex or $\mathbb{A}^{1}$ if it has two, see lemma 9.2). From the explicit description of $\mathbb{P}^{G}$ as a blow-up, one can check that $D^{(2)}$ is a mixed Tate motive over $\mathbb{Q}$ unramified at all primes by applying the criterion of [35], proposition 4.3. It therefore has no non-trivial periods. It follows that the only possible periods in weight $\leq 2$ are rational numbers (periods of $\mathbb{Q}(0)$ ), and rational multiples of the Lefschetz motivic period $\mathbb{L}^{\mathfrak{m}}$, whose period is $2 \pi i$. The latter is anti-invariant under the action of the real Frobenius involution. On the other hand, motivic periods of graphs are invariant under the real Frobenius, since the Betti class $\sigma_{G}$ is fixed under its action. This rules out the second case.

Since a motivic $\operatorname{logarithm} \log ^{\mathfrak{m}}(x)$ is determined by its period $\log (x)$, an alternative approach to proving this lemma would be by direct computation of the periods of the two and three-edge graphs, along the lines of the method of Appendix 2.

This innocuous-looking statement provides a constraint to all orders in perturbation theory, i.e., an equation satisfied by all motivic Feynman amplitudes.

Theorem 9.4. Let $G$ be a primitive log-divergent graph, and $I_{G}^{\mathfrak{m}}$ its motivic amplitude. Then every Galois conjugate which is of weight $\leq 2$ is rational.

In fact, no element of $\mathcal{F} \mathcal{P}_{0,0}^{\mathfrak{m}}$ can have a Galois conjugate of the form $\log ^{\mathfrak{m}}(p)$ for $p$ prime. This theorem is consistent with the coaction conjecture 2 below.

Example 9.5. In [46], Schnetz and Panzer give examples of amplitudes of graphs $P_{9,36}=P_{9,75}$ and $P_{9,107}=P_{9,111}$ with 9 loops which are Euler sums and are 
constrained in a non-trivial way by this theorem. One verifies by replacing them with their motivic versions (assuming the period conjecture for Euler sums) that they never have a Galois conjugate $\log ^{\mathfrak{m}}(2)$.

In a similar way, one easily checks that the motivic period $\operatorname{Li}_{2}^{\mathfrak{m}}\left(\zeta_{6}\right)$, where $\zeta_{6}$ is a primitive $6^{\text {th }}$ root of unity does not occur in $W_{4} \mathcal{F} \mathcal{P}_{0,0}^{\mathfrak{m}}$. This gives a non-trivial constraint on the amplitudes $P_{7,11}, P_{8,33}$ and $P_{9,136}=P_{9,149}$ at seven, eight and nine loops respectively. See [46] for further details.

9.4. A programme for a Galois theory of graphs. There are several known families of algebraic relations between amplitudes of graphs of type $(0,0)$. We expect that many of these relations also hold between motivic periods. This suggests the following list of relations through which the map (9.3) might factorize:

(1) (Tadpoles). $G \sim G / e$ where $e$ is a tadpole (self-edge).

(2) (Series-Parallel operations). $G \sim G^{\prime}$ where $G^{\prime}$ is obtained from $G$ by subdividing an edge or duplicating an edge.

(3) (Planar duals). $G \sim G^{\vee}$ where $G$ is planar, and $G^{\vee}$ is its dual graph.

(4) (One and two-vertex joins). If $G$ is a one or two-vertex join of graphs $G_{1}$ and $G_{2}$ then we expect a relationship between $\mathcal{F P}^{\mathfrak{m}}(G)$ and $\mathcal{F P}^{\mathfrak{m}}\left(G_{1}\right) \mathcal{F P}^{\mathfrak{m}}\left(G_{2}\right)$.

(5) (Completion and twist identities). $G \sim G^{\prime}$ where $G$ and $G^{\prime}$ are twist-related or obtained from a four-regular graph by deleting a vertex [48].

Note that (3) could be extended to non-planar graphs if one considers graph motives of matroids. If one replaces $\operatorname{mot}(G)$ with a cubical version $\operatorname{mot}_{c}(G)$ following [18], then (3) holds automatically. The series and parallel operations are (planar) dual to each other. The three-edge examples of $\S 9.3$ are equivalent to the empty graph by (2). The relation (5) is considerably more speculative than the others.

In addition, we can hope for precise information about the weights of graphs. We expect that the highest weight-graded quotient of $\mathcal{F} \mathcal{P}^{\mathfrak{m}}(G)$ should be related to the $c_{2}$-invariant of a graph, ${ }^{18}$ which is known to satisfy several further combinatorial identities. This suggests, at the very least for graphs satisfying $N_{G}=2 h_{G}$, that

(6) $w(G) \leq 2 N_{G}-6$, and $w(G) \leq 2 N_{G}-8$ if $G$ has weight-drop.

(7) $w(G) \leq 2 N_{G}-8$ if $G$ contains a sub-divergence.

(8) $w(G) \leq n$ if and only if $w\left(G^{\prime}\right) \leq n$ whenever $G, G^{\prime}$ are equivalent under double-triangle reduction.

It is highly likely that there are further relations between motivic periods of graphs which have three-valent vertices or triangles, which remain to be discovered. ${ }^{19}$

Finally, one would like to have some control on the degree of unipotency of motivic periods. This has not been investigated.

9.5. Coaction conjecture and speculation. Recall that a graph $G$ of type $(0,0)$ is said to be in $\phi^{n}$ if every vertex of $G$ has degree at most $n$.

Definition 9.6. Let $\mathcal{F P}_{\phi^{4}}^{\mathfrak{m}} \subset \mathcal{F} \mathcal{P}_{0,0}^{\mathfrak{m}}$ denote the $\mathbb{Q}$-vector space spanned by the motivic amplitudes (9.2) of primitive log-divergent graphs in $\phi^{4}$ theory.

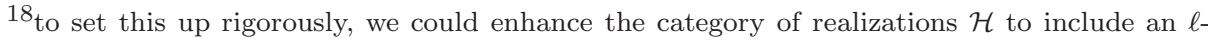
adic component. The $c_{2}$-invariant should be obtained from the action of Frobenius on the highest non-trivial weight-graded piece of $\left(\operatorname{mot}_{G}\right)_{\ell}$.

19 we know, for example, that graphs of vertex width $\leq 3$ evaluate to multiple zeta values but the numbers of such graphs greatly exceeds the dimension of the space of multiple zeta values of the appropriate weight, so there must exist many relations between these amplitudes.
} 
The following extraordinary conjecture was formulated in [46] and called the coaction conjecture. It was a principal motivation for the present paper.

Conjecture 2. [46] $\mathcal{F} \mathcal{P}_{\phi^{4}}^{\mathfrak{m}}$ is stable under the action of $C_{0,0}$.

This conjecture goes far beyond what we can presently prove, but has been verified numerically in hundreds of examples [46]. However, proving any or all of the properties of $\$ 9.4$ would lead to spectacular consequences for graph amplitudes in the direction of this conjecture. For example, let us assume only (2), and call two graphs sp-equivalent if they are obtained from each other by series-parallel operations. The smallest graph not equivalent to the trivial graph is the wheel with three spokes $W_{3}$ with six edges (whose amplitude is $6 \zeta(3)$; we expect that $\zeta^{\mathfrak{m}}(3)$ is its unique non-trivial motivic period) followed by the wheel with four spokes $W_{4}$ with eight edges. By stability, we would deduce that

$$
W_{7} \mathcal{F} \mathcal{P}_{0,0}^{\mathfrak{m}}=\mathbb{Q} \oplus \zeta^{\mathfrak{m}}(3) \mathbb{Q} \text {. }
$$

In particular, no $\zeta^{\mathfrak{m}}(2)$ occurs, which would then imply that no motivic period at any loop order can have a Galois conjugate $\zeta^{\mathfrak{m}}(2)$. Since $W_{3}$ is primitive logdivergent in $\phi^{4}$ this would also prove conjecture 2 up to weight seven:

$$
W_{n} \mathcal{F} \mathcal{P}_{\phi^{4}}^{\mathfrak{m}}=W_{n} \mathcal{F} \mathcal{P}_{(0,0)}^{\mathfrak{m}} \quad \text { for } n \leq 7,
$$

since the right-hand side is stable under the action of $C_{0,0}$ by definition. Things become interesting at nine edges. There are three non-trivial sp-equivalence classes of graphs: the graph obtained by deleting an edge from the complete graph $K_{5}$; the complete bipartite graph $K_{3,3}$; and a planar graph which is the skeleton of triangular prism. By continuing in this manner, and computing motivic periods of ever-larger graphs, one could deduce infinite families of constraints on motivic periods of Feynman graphs to all orders. This would go a long way to explain the remarkable structure observed in [46].

Example 9.7. To illustrate how the topology of graphs can impinge upon their periods, consider any class of graphs $\mathfrak{C}$ which is stable under edge contraction and motic subgraphs. It defines a subspace $\mathcal{F P}_{\mathfrak{C}}^{\mathfrak{m}} \subset \mathcal{F P}_{(0,0)}^{\mathfrak{m}}$ which is stable under the action of the constant cosmic Galois group $C_{0,0}$. For example, the motivic periods of planar graphs are Galois-stable.

Specialising further still, consider the following thought-experiment for the wheel with $n$-spokes graphs $W_{n}$. Since there are several missing elements we shall be very brief. The key topological property of the wheel graphs is that, modulo spequivalence, the vector space they span is stable under motic descendants. Another way to say this is contracting an edge in a wheel leads to a graph which is either sp-equivalent to another wheel, or the trivial graph. Every motic subgraph of a wheel has the same property.

Bloch-Esnault-Kreimer have proved that (e.g., as an object of $\mathcal{H}$ ),

$$
H^{2 n-1}\left(\mathbb{P}^{2 n-1} \backslash X_{W_{n}}\right) \cong \mathbb{Q}(3-2 n) .
$$

Let us assume (2) of $\S 9.4$ and furthermore that the wheel motives are mixed Tate over $\mathbb{Z}$ and have no non-trivial non-global motivic periods (i.e., the conclusion, but not necessarily the hypotheses of proposition 10.7 in [17] hold). This would imply that all elements in $(\sigma \operatorname{mot}(G))_{d R}$ are images of classes $(9.4)$ via face maps and hence have weights $\equiv 2(\bmod 4)$. By the following proposition,

$$
\mathcal{F} \mathcal{P}^{\mathfrak{m}}\left(W_{n}\right)=\mathbb{Q} \oplus \mathbb{Q} \zeta^{\mathfrak{m}}(3) \oplus \mathbb{Q} \zeta^{\mathfrak{m}}(5) \oplus \ldots \oplus \mathbb{Q} \zeta^{\mathfrak{m}}(2 n-3) \text {. }
$$


In particular, this would imply that all periods of the wheels graphs are linear combinations of odd zeta values only. It is known that the wheel amplitude (9.2) itself is an explicit rational multiple of a single odd zeta value of highest weight. In this case, therefore, we expect to see a direct relationship emerging between the topological properties of wheel graphs in the motic Hopf algebra and the Galoistheoretic properties of their periods.

Proposition 9.8. Let $M$ be an effective mixed Tate motive over $\mathbb{Z}$ such that

$$
\operatorname{gr}_{4 n}^{W} M=0 \quad \text { for all } n \geq 1 .
$$

Then the real (i.e., Frobenius-invariant) motivic periods of $M$ are $\mathbb{Q}$-linear combinations of 1 and $\zeta^{\mathfrak{m}}(2 n+1)$, for $n \geq 1$.

Proof. Choose generators $\sigma_{2 n+1}$ of the de Rham graded Lie algebra of $\mathcal{M T}(\mathbb{Z})$ in odd degrees $-2 n-1$ for $n \geq 1$, where the degree is the 'MZV-weight', or one half of the Hodge-theoretic weight. The non-rational motivic periods of $M$ have only odd degrees by assumption. Consider a motivic period $\xi_{2 m+1}$ of $M$ of degree $2 m+1>0$. Since $\sigma_{2 n+1} \xi_{2 m+1}$ has even degree $2(n-m)$, it is zero unless $n=m$ and $\sigma_{2 n+1} \xi_{2 m+1} \in \mathbb{Q}$. Therefore $\xi_{2 n+1}$ has de Rham Galois conjugates itself and 1. It is therefore primitive (unipotency degree $\leq 1$ ). By theorem 3.3 of $[15]$ it is in the space $\zeta^{\mathfrak{m}}(2 n+1) \mathbb{Q} \oplus\left(\mathbb{L}^{\mathfrak{m}}\right)^{2 n+1} \mathbb{Q}$. Since it has real periods and $\operatorname{per}\left(\mathbb{L}^{\mathfrak{m}}\right)=2 i \pi$ is imaginary, it is a rational multiple of $\zeta^{\mathfrak{m}}(2 n+1)$.

Remark 9.9. The previous argument fails for a weight-drop graph such as the bipartite graph $K_{3,4}$, since its motive is non-trivial in weight $16 \equiv 0(\bmod 4)$ and the previous proposition does not apply. It has motic graph sub-quotients $W_{3}$ and $W_{4}$, so the same argument would allow the motivic amplitude of $K_{3,4}$ to have Galois conjugates $\zeta^{\mathfrak{m}}(3)$ and $\zeta^{\mathfrak{m}}(5)$. This is entirely consistent with the fact that

$$
I_{K_{3,4}}=-\frac{216}{5} \zeta(5,3)-81 \zeta(5) \zeta(3)+\frac{522}{5} \zeta(8)
$$

which, assuming the period conjecture for multiple zeta values, indeed has nontrivial Galois conjugates $\zeta(3)$ and $\zeta(5)$.

In conclusion, the Galois theory of graphs described here, with a few extra speculative ingredients such as those outlined in $\S 9.4$, seems to predict quite accurately the observed patterns of periods in amplitude computations at low loops.

\section{EXAMPLES WITH GENERAL KINEMATICS AND CONJECTURES}

10.1. General kinematics. It is possible to undertake a classification of the motivic periods of graphs with few edges and arbitrary external kinematics as above. By the small graphs principle and Galois action, it leads, in the same way as $\S 9.3$, to all order constraints on Feynman periods of any type $(Q, M)$. We shall not discuss this here, but only make a few brief comments.

First of all, the cohomology of generic one-loop graphs was studied in [11] and can be re-expressed in the language of motivic periods. Applying formula (7.2) it provides a computation of the motivic coaction. The case of graphs with subdivergences can be treated using the techniques described here, and one example is treated in full detail in an appendix $\S 12$. The recent preprints [1] and [2] give conjectural formulae for the coaction on some examples of graphs (with the caveat that one side of the coaction needs to be expressed in terms of de Rham periods). 
I expect that these formulae can be proved from first principles using the cohomological techniques described here. A very interesting observation of loc. cit. is that the coaction formulae apparently continue to hold on the level of $\varepsilon$-expansions in dimensional regularisation.

Note also that a complete analysis of Feynman graphs with up to three edges would include the sunrise graph, which involves the cohomology of a family of elliptic curves and has a very extensive literature. The results of [14] likewise can be used to deduce information about the corresponding motivic periods .

Remark 10.1. Other interesting classes of graphs to study in this framework are those of type $(Q, M)=(2,0)$ or $(0,1)$ which depend on a single scale. When the scale factorizes out of the graph polynomials, the corresponding amplitudes effectively depend upon a single number. The massive banana graphs (see [12]), for example, would seem to generate a small family of motivic periods which are stable under the cosmic Galois group, and hence should have interesting arithmetic properties. We suspect that this could explain why certain combinations of periods related to banana graphs are periods of pure motives, and hence, by Deligne's conjecture, are critical values of the underlying $L$-functions.

10.2. Graphs with many external legs. The parametric representation is inefficient for graphs with many edges, and the number of edges does not accurately predict the expected weight of the amplitude. The existence of the momentum space representation suggests the weight depends on the loop number.

Conjecture 3. Let $G$ be a Feynman graph with $h$ loops, in $d \in 2 \mathbb{N}$ space-time dimensions, and let $\omega_{G}$ be the integrand of the Feynman amplitude. Then

$$
\omega_{G} \in \omega_{d R}^{\mathrm{gen}} W_{d h} \operatorname{mot}_{G}
$$

Remark 10.2. This conjecture only gives a bound on the weights of amplitudes, and not general Feynman periods, which could potentially have higher weights.

The heuristic rationale behind the conjecture is as follows:

- There should exist objets $\operatorname{mot}_{G}^{\mathrm{mom}}$ in a category of realisations such that the amplitude in momentum space is the period of $\left[\operatorname{mot}_{G}^{\mathrm{mom}}, \omega_{G}^{\mathrm{mom}}, \sigma_{G}^{\mathrm{mom}}\right]^{\mathfrak{m}}$.

- The Schwinger trick (universal quadric) should give an equivalence

$$
\left[\operatorname{mot}_{G}^{\mathrm{mom}}, \omega_{G}^{\mathrm{mom}}, \sigma_{G}^{\mathrm{mom}}\right]^{\mathfrak{m}}=\left[\operatorname{mot}_{G}, \omega_{G}, \sigma_{G}\right]^{\mathfrak{m}}
$$

This was partly carried out in [10], equation (10.4), in the case of no kinematics or subdivergences, and for the absolute (not relative) cohomology.

- The momentum space integrand should satisfy $\omega_{G}^{\text {mom }} \in W_{d h}\left(\operatorname{mot}_{G}^{\operatorname{mom}}\right)_{d R}$.

This conjecture, combined with the stability conjecture 1, would yield powerful identities for Feynman amplitudes. In particular, it suggest the following.

Conjecture 4. Let $G$ be as in conjecture 3. The amplitude of $G$ is a (regularised) period of the motic descendants of $G$ of degree $\leq d h$.

Let $d=4$. Since, for every $h$ there are only finitely many graph topologies of degree $\leq d h$, this would give a finite set of 'master integrals' for graphs with arbitrarily many external legs, at every loop order.

Examples 10.3. Conjecture 3 is certainly true for one-loop graphs. Let $G$ be such a graph. Then its Feynman amplitude is of weight $\leq 4$. It is expressible as 
a Feynman period of quotients of $G$ with at most five edges (in this case one can do slightly better and replace 'five' with 'three'). This is a theorem due to Nickel [43], reproved in [11], and can be made effective. The analogue of this theorem for graphs with two loops is not presently known, it seems, and the programme outlined above suggests a generalisation to all higher loop orders.

10.3. Further directions. Some directions for further research include:

(1) One would like to incorporate ultraviolet divergent graphs and the theory of renormalisation along the lines of [21]. Since the geometry of the Feynman polytope is very close to the BPHZ forest formula, the theory of renormalisation fits very naturally in the present framework. One approach, which is closest to that used in physics, would be to allow integrals with logarithmic terms in the numerators. This can be done by defining a notion of motivic periods with coefficients (one needs to interpret an integral of a family of motivic periods ${ }^{20}$ as a motivic period).$^{21}$ Another approach, which is perhaps less satisfactory, is to differentiate with respect to a scale in order to turn all integrands into algebraic differential forms, as in [21]. Indeed, one can define the graph motives of UV divergent graphs simply by using the decomposition into angles and scales of [21] and taking the renormalised graph motive defined there.

(2) It would be interesting for applications to understand situations with infrared singularities when the genericity assumptions (1.17) are not satisfied. The graph factorisation theorems partially break down in this case, but by enlarging the class of polynomials considered, one might still retrieve a Galois theory of graphs. The QED contributions to the anomalous magnetic moment $g-2$ are a fascinating case study.

(3) One would like to rethink the problems of resummation of the perturbative expansion in the context of motivic periods. Taking a sum of amplitudes viewed merely as complex numbers ignores the fact that they are periods and all the structure that that entails. We expect the perturbative expansion can be lifted canonically to a series whose coefficients are motivic periods. The invariants of motivic periods defined in [17] should enable one to sum this perturbative series in a more organised manner, e.g., according to various types, which may lead to better convergence properties.

(4) In our theory, the domain of integration is trivial and all the content of the physical theory is in the integrand. For this reason the de Rham Galois group plays a privileged role. The remarks in this chapter also suggest that the graph motive in parametric space is not optimal (at least for graphs with many edges), and one must also consider momentum space or other integral representations, which will give a different bound on the space of Galois conjugates of amplitudes. It seems to be an important fact that amplitudes have several quite different integral representations, each giving different constraints on their Galois theory.

(5) We worked exclusively in Euclidean space. In order to analytically continue to Minkowski space, one would like to know where the singularities of the Feynman integrals are. A worrying possibility is that the set of singularities

\footnotetext{
${ }^{20}$ For example, one can make sense of a formula of the form $\zeta^{\mathfrak{m}}(2)=-\int_{0}^{1} \log { }^{\mathfrak{m}}(1-x) \frac{d x}{x}$

${ }^{21}$ This also seems to be a possible way to study dimensional regularisation: the coefficients of a Taylor expansion in $\varepsilon$ are integrals with logarithmic numerators.
} 
of graphs of a fixed type $(Q, M)$ could become dense in the space $K_{Q, M}$ as the loop number increases. This is why our de Rham fiber functor is at the generic point. It would be interesting to know if there is an open region in kinematic space where all Feynman amplitudes are non-singular.

(6) There is good evidence to suggest that superstring amplitudes have a Galois theory of their own (at least at tree-level [47]). This seems entirely reasonable given that the moduli spaces $\mathfrak{M}_{g, n}$ have the same product-structure on their stratification as the one exploited here for amplitudes.

\section{Appendix I: SOME COHOMOlOGICAL TOOLS FOR PERIOdS}

Not every cohomology class in the de Rham realisation of the graph motive can be represented by a global differential form such as (6.13). A study of nonglobal periods is necessary for understanding the conjugates of amplitudes under the cosmic Galois group. Therefore in this section we provide some tools for studying such non-global cohomology classes and their periods. The first is a complex which we use to show that the periods of graphs are limits of divergent integrals of globallydefined forms. The second is a spectral sequence which allows us to import known results about the cohomology of graph hypersurfaces in projective space to the study of graph motives. It is related to the motic Hopf algebra.

11.1. A relative algebraic Čech-de Rham complex. Let $D \subset X$ be a simple normal crossing divisor in a smooth scheme $X$ over $\mathbb{Q}$. Let $U_{i} \subset X$, for $i \in I$, be a covering of $X$ by a finite collection of smooth affine varieties defined over $\mathbb{Q}$. Let $D_{j}$, for $j \in J$, denote the irreducible components of $D$. Write as usual $U_{P}=\cap_{i \in P} U_{i}$ for $\emptyset \subsetneq P \subset I$ and $D_{Q}=\cap_{i \in Q} D_{i}$ for $Q \subset J$ with the convention $D_{\emptyset}=X$.

Consider the triple complex

$$
\Omega^{n, p, q}\left(\left\{U_{i}\right\}, D\right)=\bigoplus_{|P|=p,|Q|=q} \Omega^{n}\left(U_{P} \cap D_{Q}\right)
$$

where $\Omega^{n}\left(U_{P} \cap D_{Q}\right)$ denotes the global sections of the sheaf of Kahler differential forms over $\mathbb{Q}$. The differentials $\Omega^{n, p, q}\left(\left\{U_{i}\right\}, D\right) \rightarrow \Omega^{n+1, p, q}\left(\left\{U_{i}\right\}, D\right)$ are given by the usual differential $d$ in the de Rham complex. The differentials $\Omega^{n, p, q}\left(\left\{U_{i}\right\}, D\right) \rightarrow$ $\Omega^{n, p+1, q}\left(\left\{U_{i}\right\}, D\right)$ are given by the differentials in the usual Cech complex, and the differentials $\Omega^{n, p, q}\left(\left\{U_{i}\right\}, D\right) \rightarrow \Omega^{n, p, q+1}\left(\left\{U_{i}\right\}, D\right)$ are given by restriction of differential forms to closed subsets $D_{Q \cup\{q\}} \subset D_{Q}$ with the standard sign convention. The relative algebraic de Rham cohomology

$$
H_{d R}^{n}(X, D)=H^{n}\left(\operatorname{Tot}\left(\Omega^{n, p, q}\left(\left\{U_{i}\right\}, D\right)\right)\right.
$$

is the cohomology of the total complex associated to the triple complex (11.1). A cohomology class of degree $n$ in the latter can be represented by a collection

$$
\omega_{Q}^{P} \in \Omega^{n+1-p-q}\left(U_{P} \cap D_{Q}\right)
$$

where $P \subset I$ and $Q \subset J$ with $|P|=p,|Q|=q$ that are mapped to zero by the total differential. Associated to the triple complex (11.1) are a number of spectral sequences, for example

$$
E_{1}^{p, q}=\bigoplus_{|P|=p+1} H_{d R}^{q}\left(U_{P}, U_{P} \cap D\right) \quad \Longrightarrow \quad H_{d R}^{p+q}(X, D)
$$

where the differential is induced by inclusions as for the Čech complex. 
11.2. A relative Stokes' theorem. Let $C_{n}\left(U_{P} \cap D_{Q}\right)$ denote the complex of singular $n$-chains with coefficients in $\mathbb{Q}$ on the topological space $U_{P} \cap D_{Q}(\mathbb{C})$. By analogy with (11.1), define a triple complex of singular chains

$$
C_{n, p, q}\left(\left\{U_{i}\right\}, D\right)=\bigoplus_{|P|=p,|Q|=q} C_{n}\left(U_{P} \cap D_{Q}\right)
$$

where the differentials are given by the boundary map on chains, and the inclusion maps, with the appropriate signs. The homology of the total complex is the relative Betti homology

$$
H_{B}^{n}(X, D)^{\vee}=H_{n}(X(\mathbb{C}), D(\mathbb{C}))=H_{n}\left(\operatorname{Tot}\left(C_{n, p, q}\left(\left\{U_{i}\right\}, D\right)\right)\right) .
$$

A relative homology class can be represented by a collection of chains

$$
\sigma_{Q}^{P} \in C_{n+1-p-q}\left(U_{P} \cap D_{Q}\right)
$$

whose total differential is zero. Denote such a collection by $\sigma=\left\{\sigma_{Q}^{P}\right\}$.

Given such a chain of degree $n$, and a cohomology class $\omega \in H_{d R}^{n}(X, Z)$ represented by a collection (11.2), define the period (or integration pairing) by

$$
\int_{\sigma} \omega:=\sum_{P, Q} \int_{\sigma_{Q}^{P}} \omega_{Q}^{P} .
$$

Note that there can be signs in this formula depending on sign conventions for the differentials in the complexes defined earlier. These are not important for the general discussion which follows. The following theorem is a corollary of Grothendieck's theorem [37]. I was unable to find a suitable reference in the literature. ${ }^{22}$

Theorem 11.1. The pairing (11.5) is well-defined and computes the isomorphism

$$
\operatorname{comp}_{B, d R}: H_{d R}^{n}(X, D) \otimes_{\mathbb{Q}} \mathbb{C} \stackrel{\sim}{\longrightarrow} H_{B}^{n}(X, D)^{\vee} \otimes_{\mathbb{Q}} \mathbb{C} .
$$

Proof. (Sketch). The pairing is well-defined by Stokes' formula, along with the definition of the differentials in the complexes (11.1) and (11.3). By some standard homological algebra, the result follows from Grothendieck's algebraic de Rham theorem for affine varieties, which implies that integration defines a natural isomorphism $H_{d R}^{i}\left(U_{P} \cap D_{Q}\right) \otimes_{\mathbb{Q}} \mathbb{C} \stackrel{\sim}{\longrightarrow} H_{B}^{i}\left(U_{P} \cap D_{Q}\right) \otimes_{\mathbb{Q}} \mathbb{C}$ for all $P, Q$.

11.3. Sectors and blow-ups in projective space. We can apply the above to the following situation. With the notation of $\S 5$, let $S$ be a finite set and $B \subset 2^{S}$ be a set of subsets of $S$ closed under unions. Let $P^{B}$ denote the corresponding blow-up of $\mathbb{P}^{S}$, and $D^{B} \subset P^{B}$ the normal crossing divisor defined in (5.2). Let $Y \subset P^{B}$ be a closed subvariety with the property that $Y \cap \widetilde{\sigma}_{B}=\emptyset$. We set

$$
X=P^{B} \backslash Y \quad \text { and } \quad D=D^{B} \backslash\left(D^{B} \cap Y\right) .
$$

The spaces $P^{B}$ come with a natural affine covering $\left\{U_{\mathcal{F}, c}\right\}=\left\{\mathbb{A}^{\mathcal{F}, c} \backslash\left(\mathbb{A}^{\mathcal{F}, c} \cap Y\right)\right\}$ where the $\mathbb{A}^{\mathcal{F}, c}$ are isomorphic to affine spaces $\mathbb{A}^{n}$. We have in mind, of course, the case where $B$ is the set of motic subgraphs of a Feynman graph, and $Y$ the strict transform of graph hypersurfaces.

The polytope $\widetilde{\sigma}_{B}$ defined in $\S 5.2$ can be decomposed into regions in the following way. Choose any point which lies in the interior of $\sigma$ :

$$
z=\left(z_{1}: \ldots: z_{n}\right) \in \mathbb{P}^{S}(\mathbb{R}) \quad \text { where } z_{i}>0 \text { for all } i .
$$

\footnotetext{
${ }^{22}$ Although, whilst writing up these notes, Huber and Müller-Stach kindly sent me a preliminary draft of their book project on periods, which contains similar considerations.
} 
It defines a point on every open $U_{\mathcal{F}, c} \subset \mathbb{A}^{\mathcal{F}, c}$ in our covering. Let $\beta_{1}, \ldots, \beta_{n}$ be the coordinates on $\mathbb{A}^{\mathcal{F}, c}$ defined by (5.9). Then the inverse image of $z$ is given by equations $\beta_{i}=z_{a_{i}} / z_{b_{i}}$ for some indices $a_{i}, b_{i}$. The equations $\beta_{i}=0$ and $\beta_{i}=z_{a_{i}} / z_{b_{i}}$ define a hypercube $H_{z}$ in $U_{\mathcal{F}, c}$. Let $\sigma_{B}^{A}(z)$ be a face of this hypercube:

$$
\sigma_{B}^{A}(z):=\left\{\left(\beta_{1}, \ldots, \beta_{n}\right) \in H_{z}: \beta_{i}=0 \text { for } i \in A, \beta_{i}=z_{a_{i}} / z_{b_{i}} \text { for } i \in B\right\},
$$

where $A, B \subset\{1, \ldots, n\}$ are disjoint. One can verify that $\sigma$ is tessellated by a set of $\sigma_{Q}^{P}(z)$, over the different charts $\mathcal{F}, c$, for certain sets $P, Q$, and that $\left\{\sigma_{Q}^{P}(z)\right\}$ defines a relative Betti homology class representing $\sigma$.
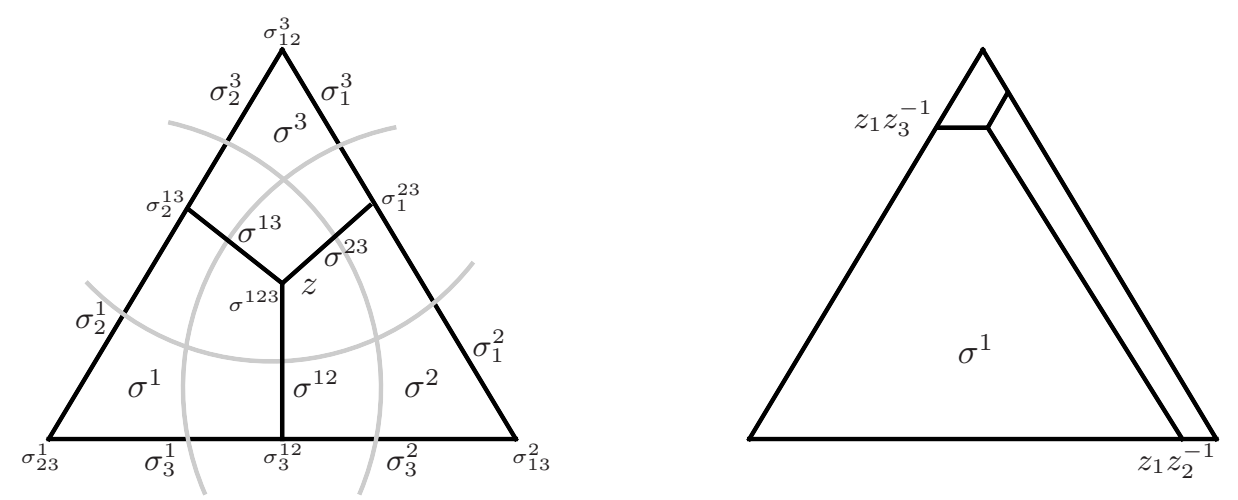

FiguRE 1. A decomposition of the coordinate simplex in $\mathbb{P}^{3}$ (here $B=\emptyset)$, defined by hyperplanes $z_{i} \alpha_{i}=z_{j} \alpha_{j}$ for $0<z_{i}<\infty$ and $i=1,2,3$. The affine open sets $U_{i}: \alpha_{i} \neq 0$ for $i=1,2,3$ are depicted schematically by grey arcs. On the right, $z_{2}, z_{3} \rightarrow 0$.

Corollary 11.2. Any relative period of $P^{B} \backslash Y$ over the domain $\sigma$ is given by

$$
\int_{\sigma} \omega=\sum_{P, Q} \int_{\sigma_{Q}^{P}(z)} \omega_{Q}^{P},
$$

where the $\omega_{Q}^{P}$ are differential forms (11.2) on an affine $U^{P} \cap D_{Q}$.

This corollary gives a means, albeit an inefficient one, to compute non-global Feynman periods. It is adapted to the method of parametric integration [44]. For instance, letting all $z_{i}=1$, each term in the sum in local coordinates is an integral of an algebraic differential form over a cube $[0,1]^{m}$ for some $m \leq n$.

Remark 11.3. This procedure is not to be confused with the notion of sector decomposition in the physics literature. In that setting, one integrates the (pull-back of) the same globally-defined form $\omega$ over each sector $\sigma^{P}$ and sums the contributions. In the above, we are integrating different forms $\omega_{Q}^{P}$ over each sector.

11.4. Limits and regularisation. The integral (11.7) does not depend on the point $z$. Since each open affine $U_{i}(\mathbb{C})$ contains the preimage of $z$ for any point $z$ (11.6) in the interior of the coordinate simplex $\sigma$, we can take limits in (11.7):

$$
\int_{\sigma} \omega=\lim _{z \rightarrow \infty} \sum_{P, Q} \int_{\sigma_{Q}^{P}(z)} \omega_{Q}^{P}
$$


as $z$ tends to any point on the boundary of $\sigma$. Many of the terms in the sum on the right-hand side will tend to zero and can be dropped. By repeatedly taking limits, one obtains an expression for the integral on the left-hand side as limits of possibly divergent integrals over facets of $\sigma_{B} \cdot{ }^{23}$

11.5. The exceptional locus spectral sequence. In this section, let $X, D$ denote fibres of $X, D$ as defined in $\S 11.1$. For reasons which will become apparent in a moment, let us write $E=D$. The divisor $E$ defines a stratification on $X$ by closed subvarieties. Write

$$
E_{J}^{o}=E_{J} \backslash\left(E_{J} \cap \bigcup_{j \notin J} E_{j}\right) .
$$

For instance, $E_{\emptyset}^{o}=X \backslash E$. There is a 'Gysin' or residue spectral sequence

$$
E_{1}^{p, q}=\bigoplus_{|J|=p} H^{q-p}\left(E_{J}^{o}\right)(-p) \quad \Longrightarrow \quad H^{p+q}(X)
$$

where the differentials $d_{1}$ are given by residues along the irreducible components of $E$ and $p, q \geq 0$. From now on, let $X=P^{G} \backslash Y_{G}$ where $G$ is a Feynman graph, and let $E=D$ be defined by (5.2). If $G$ has no masses or momenta, we obtain the spectral sequence considered by Bloch in [9]. The open strata $E_{J}^{o}$ are complements of graph hypersurfaces in $\mathbb{G}_{m}^{n}$. This spectral sequence is hard to control since the cohomology of the latter is large and there are many cancellations.

Returning now to the case of a general Feynman graph $G$, and $X=P^{G} \backslash Y_{G}$, we see that it is more economical to take $E \subset D$ to be the exceptional divisor:

$$
E=\cup_{\gamma \subset G} D_{\gamma}
$$

where the union is only over the set of motic subgraphs of $G$. We shall call the corresponding spectral sequence (11.8) the exceptional locus spectral sequence.

Theorem 11.4. In this situation, the non-empty strata $E_{J}$ are indexed by strictly increasing sequences of motic subgraphs of $G$ :

$$
J: \quad \gamma_{1} \subsetneq \gamma_{2} \subsetneq \cdots \subsetneq \gamma_{r} .
$$

If we write $\gamma_{i}^{\prime}=\gamma_{i} / \gamma_{i-1}$ for the successive quotients, where $\gamma_{0}$ denotes the empty graph, then there is a canonical isomorphism

$$
E_{J}^{o} \cong\left(\mathbb{P}^{N_{\gamma_{1}^{\prime}}-1} \backslash X_{\gamma_{1}^{\prime}}\right) \times \cdots \times\left(\mathbb{P}^{N_{\gamma_{r}^{\prime}}-1} \backslash X_{\gamma_{r}^{\prime}}\right)
$$

When $J=\emptyset$ is the empty set, $E_{\emptyset}^{o} \cong \mathbb{P}^{N_{G}-1} \backslash X_{G}$. Therefore the $E_{1}$ terms of the spectral sequence (11.8) only involve the cohomology of graph hypersurface complements in projective space of quotients of motic subgraphs of $G$. In particular,

$$
E_{1}^{p, q}=0
$$

if $q \geq N_{G}$ or if $p \geq h_{G}+1$. If $G$ has no masses or momenta, $E_{1}^{p, q}=0$ for $p \geq h_{G}$.

\footnotetext{
${ }^{23}$ It would be interesting, by studying the asymptotic behaviour of these integrals as $z$ tends to the boundary, to define a consistent notion of regularisation of divergent integrals over faces of the polytope $\sigma_{B}$ which commutes with these limits. In this case, we could write the period of any non-global form as a linear combination of regularised integrals of global forms over faces of $\sigma_{B}$. There is no shortage of regularisation techniques for Feynman integrals in the physics literature. For instance, if $P^{B}=P^{G}$ is obtained from a Feynman graph, and $Y_{G}$ the graph hypersurface, the boundary strata of $P^{G}$ are related to the motic Hopf algebra, and closely resembles the combinatorics of the BPHZ forest formula (see [21]). This suggests a possible way to renormalise divergent integrals using the subtraction of counter-terms.
} 
Proof. By theorem 5.1 and the fact that the union of two motic subgraphs is motic, two irreducible components $E_{\gamma_{1}}, E_{\gamma_{2}}$ of $E$ meet if and only if $\gamma_{1}, \gamma_{2}$ are nested. Iterating, we see that every $E_{J}$ corresponds to a nested sequence of motic graphs (11.9), and furthermore, by applying theorem 6.5, that

$$
E_{J} \cong\left(P^{\gamma_{1}^{\prime}} \backslash Y_{\gamma_{1}^{\prime}}\right) \times \ldots \times\left(P^{\gamma_{r}^{\prime}} \backslash Y_{\gamma_{r}^{\prime}}\right)
$$

Now every divisor $E_{j}$ with $j \notin J$ which meets $E_{J}$ corresponds to a motic subgraph $\gamma \subset G$ such that $\gamma_{i-1} \subsetneq \gamma \subsetneq \gamma_{i}$ for some $i$. The latter are in one-to-one correspondence with the motic subgraphs of $\gamma_{i}^{\prime}=\gamma_{i} / \gamma_{i-1}$ by theorem 3.6. Therefore $E_{J}^{0}$ is obtained from $E_{J}$ by removing all the exceptional divisors in each factor, which gives (11.10).

That $E_{1}^{p, q}=0$ for $q \geq E_{G}$ is a consequence of the fact that $\mathbb{P}^{N_{\gamma_{i}^{\prime}}-1} \backslash X_{\gamma_{i}^{\prime}}$ is affine of dimension $N_{\gamma_{i}^{\prime}}-1$, since $X_{\gamma_{i}^{\prime}}$ is a non-empty hypersurface by lemmas 1.11 and 1.13, and hence $H_{B / d R}^{r}\left(\mathbb{P}^{N_{\gamma_{i}^{\prime}}-1} \backslash X_{\gamma_{i}^{\prime}}\right)=0$ for $r \geq N_{\gamma_{i}^{\prime}}$. Finally $E_{1}^{p, q}=0$ whenever $p+1$ is strictly larger than the maximal length of any chain (11.9). This is $h_{G}+1$ if $G$ has kinematics, and $h_{G}$ otherwise by lemma 4.4 .

The terms (11.10) are in one-to-one correspondence with the terms in the $r$-fold iteration of the reduced motic coproduct.

The previous theorem implies that the graph motives defined here are extensions of the cohomology of the complements of graph hypersurfaces in projective space.

Remark 11.5. There are some variants. Firstly, if $G$ has a motic subgraph $\gamma$ with exactly one edge $e$, then the graph hypersurface has an irreducible component $V\left(\alpha_{e}\right)$. If we remove all such components from $X_{G}$ then we can consider the smaller spectral sequence using $E=\cup_{\gamma} D_{\gamma}$, where $\gamma$ are motic subgraphs of $G$ with $\geq 2$ edges. Finally, there is an obvious variant on replacing $X_{G}$ but $X_{G}^{\prime}$.

\section{APPENDIX II: WORKED EXAMPLE}

For the benefit of physicists who may not be accustomed to the techniques of the previous section, we give a complete worked example in a simple situation. The amplitudes computed here can be obtained directly, but we shall use completely general methods without taking any shortcuts, except in the very final section. Consider the graph

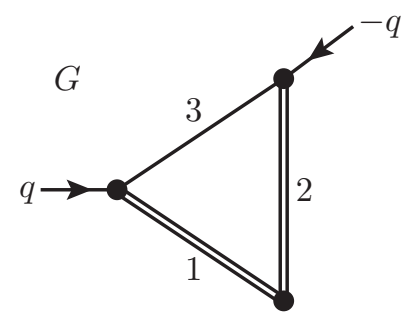

Its graph polynomial is

$$
\Xi_{G}=q^{2} \alpha_{3}\left(\alpha_{1}+\alpha_{2}\right)+\left(m_{1}^{2} \alpha_{1}+m_{2}^{2} \alpha_{2}\right)\left(\alpha_{1}+\alpha_{2}+\alpha_{3}\right)
$$

whose zero locus defines a family of quadrics $X_{\Xi_{G}} \subset \mathbb{P}^{3}$. For generic values of $q, m_{1}, m_{2}$, this quadric meets the coordinate axes at a single point $\alpha_{1}=\alpha_{2}=0$ 
which corresponds to the motic subgraph of $G$ spanned by the edges 1,2 . Let $P^{G} \rightarrow \mathbb{P}^{2}$ be the blow up of $\mathbb{P}^{2}$ at the point $D_{1} \cap D_{2}$, i.e., $\alpha_{1}=\alpha_{2}=0$, and let $Y_{G} \subset P^{G}$ be the strict transform of $X_{\Xi_{G}}$ (only). The situation is depicted below.
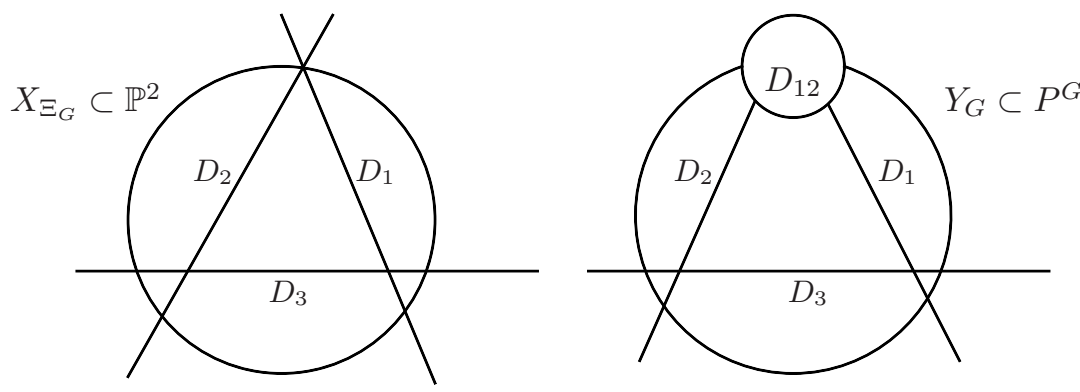

The exceptional divisor is called $D_{12}$. For simplicity, we shall only consider the graph hypersurface $X_{\Xi_{G}}$, and not $X_{\Psi_{G}}$, and compute the fibres of

$$
\operatorname{mot}_{G}^{\prime}=H^{2}\left(P^{G} \backslash Y_{G}, D \backslash D \cap Y_{G}\right)
$$

over $U_{2,2}^{\text {gen }}$, where $D$ is defined in (5.2). It satisfies $\operatorname{gr}_{2}^{W} \operatorname{mot}_{G}^{\prime} \cong \mathbb{Q}(-1) \oplus \mathbb{Q}(-1)$, and hence has two non-trivial periods. These were computed in an indirect manner in [19], §5.3.2, using the fact they are necessarily logarithms of projective invariants of seven points in $\mathbb{P}^{2}$. We give full details of the period computation using the general methods described above. The calculations are somewhat tedious but are more subtle than they may at first appear.

12.0.1. Exceptional locus spectral sequence. Let $G$ be the graph above. It has exactly one non-trivial motic subgraph $\gamma$, which is the subgraph spanned by the edges 1,2 . Since $D_{12}$ is isomorphic to a copy of $\mathbb{P}^{1}$, the relevant terms $E_{1}^{p, q}$ in the exceptional locus spectral sequence, for $(p, q) \in[0,1] \times[1,2]$, are therefore

$$
\begin{aligned}
& H^{2}\left(\mathbb{P}^{2} \backslash X_{\Xi_{G}}\right) \rightarrow H^{1}\left(\mathbb{P}^{1} \backslash X_{\Xi_{\gamma}}\right)(-1) \\
& H^{1}\left(\mathbb{P}^{2} \backslash X_{\Xi_{G}}\right)
\end{aligned}
$$

since $G / \gamma$ has only one edge and hence $\mathbb{P}^{0} \backslash X_{\Xi_{G / \gamma}}$ is a point. Since $X_{\Xi_{G}}$ is an odddimensional quadric, $H^{i}\left(\mathbb{P}^{2} \backslash X_{\Xi_{G}}\right)=0$ for $i=1,2$, and since $\Xi_{\gamma}=\left(q^{2}+m_{1}^{2}\right) \alpha_{1}+$ $\left(q^{2}+m_{2}^{2}\right) \alpha_{2}$, we have $\mathbb{P}^{1} \backslash X_{\Xi_{\gamma}}=\mathbb{A}^{1}$. Therefore the above $E_{1}^{p, q}$ terms in (12.1) are

$$
\begin{array}{ll}
0 & 0 \\
0 & \mathbb{Q}(-1)
\end{array}
$$

and hence $H^{2}\left(P^{G} \backslash Y_{G}\right)=\mathbb{Q}(-1)$. Now consider the relative cohomology spectral sequence $E_{1}^{p, q}=\bigoplus_{|I|=p} H^{q}\left(D_{I} \backslash D_{I} \cap Y_{G}\right)$ converging to $\operatorname{mot}_{G}^{\prime}$. The terms $E_{1}^{1,1}$ all vanish except for

$$
H^{1}\left(D_{3} \backslash Y_{\Xi_{G / 3}}\right)=H^{1}\left(\mathbb{G}_{m}\right) \cong \mathbb{Q}(-1)
$$

since the other faces $D_{\bullet} \backslash\left(D_{\bullet} \cap Y_{G}\right)$, for $\bullet \in\{1,2,12\}$, are copies of $\mathbb{A}^{1}$. The face $D_{3}$ is given by $\alpha_{3}=0$ and $\Xi_{G / 3}=\left(m_{1}^{2} \alpha_{1}+m_{2}^{2} \alpha_{2}\right)\left(\alpha_{1}+\alpha_{2}\right)$. Since $E_{1}^{2,0}$ is in weight zero, and by the previous computations $E_{1}^{0,2}=H^{2}\left(P^{G} \backslash Y_{G}\right)=\mathbb{Q}(-1)$, and $E_{1}^{1,2}=0$, we deduce that $\operatorname{gr}_{2}^{W} \operatorname{mot}_{G}^{\prime}=\mathbb{Q}(-1)^{\oplus 2}$. We shall compute the period of a class $[\widetilde{\omega}] \in\left(\operatorname{mot}_{G}^{\prime}\right)_{d R}$ which maps to a generator $[\omega] \in H_{d R}^{2}\left(P^{G} \backslash Y_{G}\right) \cong \mathbb{Q}(-1)$. Note that its image is zero in $H_{d R}^{2}\left(\mathbb{P}^{2} \backslash X_{\Xi_{G}}\right)=0$. The other period comes from the face $D_{3}$ via a face map, so is a period of the quotient graph $G / 3$. 
12.0.2. Affine covering. The prescription of $\S 5$ defines the following affine spaces corresponding to maximal flags of subgraphs of $G$, where $B=\{\emptyset,\{1,2\},\{1,2,3\}\}$,

$$
\begin{array}{rlll}
\mathbb{A}_{12,1}=\mathbb{A}^{\mathcal{F}, c} & \text { where } & & (\mathcal{F}, c)=\left(\emptyset \subset\{1,2\} \subset\{1,2,3\}, j_{1}=2, j_{2}=3\right) \\
\mathbb{A}_{12,2}=\mathbb{A}^{\mathcal{F}, c} & \text { where } & & (\mathcal{F}, c)=\left(\emptyset \subset\{1,2\} \subset\{1,2,3\}, j_{1}=1, j_{2}=3\right) \\
\mathbb{A}_{1}=\mathbb{A}^{\mathcal{F}, c} & \text { where } & & (\mathcal{F}, c)=\left(\emptyset \subset\{1,2,3\}, j_{1}=2\right) \\
\mathbb{A}_{2}=\mathbb{A}^{\mathcal{F}, c} & \text { where } & (\mathcal{F}, c)=\left(\emptyset \subset\{1,2,3\}, j_{1}=1\right) .
\end{array}
$$

Let $\left(\alpha_{1}: \alpha_{2}: \alpha_{3}\right)$ be projective coordinates on $\mathbb{P}^{2}$. The affine rings of the above spaces are $\mathcal{O}\left(\mathbb{A}_{12,1}\right)=\mathbb{Z}\left[\beta_{1}^{12,1}, \beta_{2}^{12,1}\right]$, where, by abuse of notation,

$$
\beta_{1}^{12,1}=\frac{\alpha_{1}}{\alpha_{2}} \quad, \quad \beta_{2}^{12,1}=\alpha_{2},
$$

and similarly $\mathcal{O}\left(\mathbb{A}_{12,2}\right)=\mathbb{Z}\left[\beta_{1}^{12,2}, \beta_{2}^{12,2}\right]$, with

$$
\beta_{1}^{12,2}=\alpha_{1} \quad, \quad \beta_{2}^{12,2}=\frac{\alpha_{2}}{\alpha_{1}},
$$

and $\alpha_{3}=1$ in both cases. Denote the coordinate rings of $\mathbb{A}_{1}$ and $\mathbb{A}_{2}$ by $\mathbb{Z}\left[\alpha_{1}, \alpha_{3}\right]$ $\left(\alpha_{2}=1\right)$ and $\mathbb{Z}\left[\alpha_{2}, \alpha_{3}\right]\left(\alpha_{1}=1\right)$ respectively. The charts $\mathbb{A} \bullet$ provide a canonical affine covering of $P^{G}$. The exceptional divisor $D_{12}$ is given by the equations $\beta_{2}^{12,1}=$ 0 and $\beta_{1}^{12,2}=0$ in the charts $\mathbb{A}_{12,1}$ and $\mathbb{A}_{12,2}$ respectively.

Let $q^{2}, m_{1}^{2}, m_{2}^{2}$ satisfy the genericity conditions (1.18), namely

$$
q^{2}+m_{1}^{2} \neq 0 \quad, \quad q^{2}+m_{2}^{2} \neq 0 \quad, \quad m_{1}^{2} \neq 0 \quad, \quad m_{2}^{2} \neq 0
$$

and for a fixed choice of such $q, m_{1}, m_{2}$, let us write

$$
\begin{aligned}
& Q=q^{2} \alpha_{3}\left(\alpha_{1}+\alpha_{2}\right)+\left(m_{1}^{2} \alpha_{1}+m_{2}^{2} \alpha_{2}\right)\left(\alpha_{1}+\alpha_{2}+\alpha_{3}\right), \\
& \bar{Q}=q^{2}\left(\alpha_{1}+\alpha_{2}\right)+\left(m_{1}^{2} \alpha_{1}+m_{2}^{2} \alpha_{2}\right)\left(\alpha_{1}+\alpha_{2}+1\right) .
\end{aligned}
$$

Let us denote by $U_{\bullet} \subset \mathbb{A}_{\bullet}$ the open subsets obtained by removing the strict transform of $V(Q)$. Thus $\mathcal{O}\left(U_{12, i}\right)=\mathbb{Z}\left[\beta_{1}^{12, i}, \beta_{2}^{12, i}, Q_{i}^{-1}\right]$ for $i=1,2$, where

$$
\begin{aligned}
& Q_{1}=q^{2}\left(\beta_{1}^{12,1}+1\right)+\left(m_{1}^{2} \beta_{1}^{12,1}+m_{2}^{2}\right)\left(\beta_{1}^{12,1} \beta_{2}^{12,1}+\beta_{2}^{12,1}+1\right) \\
& Q_{2}=q^{2}\left(\beta_{2}^{12,2}+1\right)+\left(m_{1}^{2}+m_{2}^{2} \beta_{2}^{12,2}\right)\left(\beta_{1}^{12,2} \beta_{2}^{12,2}+\beta_{1}^{12,2}+1\right)
\end{aligned}
$$

Likewise $\mathcal{O}\left(U_{1}\right)=\mathbb{Z}\left[\alpha_{1}, \alpha_{3},\left.Q\right|_{\alpha_{2}=1} ^{-1}\right]$ and $\mathcal{O}\left(U_{2}\right)=\mathbb{Z}\left[\alpha_{2}, \alpha_{3},\left.Q\right|_{\alpha_{1}=1} ^{-1}\right]$.

12.0.3. Absolute $\check{C}$ ech-de Rham class. The sets $U_{1}, U_{2}, U_{12,1}, U_{12,2}$ form our canonical open affine covering of $P^{G} \backslash Y_{G}$. Define four closed differential forms:

$$
\omega_{a b}=\left.d \log \bar{Q}\right|_{U_{12, a} \cap U_{b}} \in \Omega^{1}\left(U_{12, a} \cap U_{b}\right) \quad \text { where } a, b \in\{1,2\},
$$

where $\bar{Q}$ is defined in (12.2). Consider the element $\{\omega\}$ of degree 2 in the total complex of the absolute Cech-de Rham double complex ${ }^{24} \Omega^{n}\left(U_{P}\right)$ whose only nonzero components are the $\omega_{a b}$. The element $\{\omega\}$ is closed for the total differential and so defines a class

$$
[\omega] \in H_{d R}^{2}\left(P^{G} \backslash Y_{G}\right)
$$

\footnotetext{
${ }^{24}$ this is the triple complex (11.1) in the special case when the divisor $D$ is empty.
} 
12.0.4. Relative $\check{C}$ ech-de Rham class. We next wish to extend $\{\omega\}$ to a closed element in the relative Cech-de Rham triple complex (11.1) where $D$ has four irreducible components $D_{12}, D_{1}, D_{2}, D_{3}$. This will necessarily use the fact that $D_{i} \backslash\left(D_{i} \cap Y_{G}\right)$ is isomorphic to $\mathbb{A}^{1}$, for $i=1,2$, and hence has vanishing $H^{1}$.

Define four closed 1-forms

$$
\mu_{i} \in \Omega^{1}\left(D_{i} \cap U_{12, i}\right) \quad, \quad \nu_{i} \in \Omega^{1}\left(D_{i} \cap U_{i}\right)
$$

where $i \in\{1,2\}$, by

$$
\mu_{1}=\frac{2 m_{2}^{2} d \beta_{2}^{12,1}}{q^{2}+m_{2}^{2}\left(\beta_{2}^{12,1}+1\right)} \quad \text { and } \quad \nu_{1}=\frac{\left(q^{2}+m_{2}^{2}\right) d \alpha_{3}}{q^{2} \alpha_{3}+m_{2}^{2}\left(1+\alpha_{3}\right)}
$$

and $\mu_{2}, \nu_{2}$ are defined by interchanging all subscripts ' 1 ' and ' 2 ' (and ' 12,1 ' with ' 12,2 '). Note that $D_{i}$ does not meet $U_{12, j}$ or $U_{j}$ if $i \neq j$. Consider the element $\{\widetilde{\omega}\}$ of degree 2 in the total complex associated to (11.1) which is zero in every component of $\Omega^{n}\left(U_{P} \cap D_{Q}\right)$ except for the eight elements $\omega_{a b} \in \Omega^{1}\left(U_{12, a} \cap U_{b}\right)$ for $a, b \in\{1,2\}$ as above and $\mu_{i}, \nu_{i}$, for $i=1,2$. The element $\{\widetilde{\omega}\}$ is closed for the total differential essentially because of the equations

$$
\left.\omega_{i i}\right|_{D_{i}}=\mu_{i}-\nu_{i} \quad \text { for } i=1,2,
$$

on the open $U_{12, i} \cap U_{i} \cap D_{i} \subset D_{i}$ (recall that the left-hand side is $\left.d \log Q\right|_{\alpha_{i}=0}$ ). This defines a class in relative de Rham cohomology

$$
[\widetilde{\omega}] \in H_{d R}^{2}\left(P^{G} \backslash Y_{G}, D \backslash\left(D \cap Y_{G}\right)\right.
$$

whose image in absolute de Rham cohomology is $[\omega] \in H_{d R}^{2}\left(P^{G} \backslash Y_{G}\right)$.

12.0.5. The period. We can compute the period

$$
I=\int_{\sigma_{G}}\{\widetilde{\omega}\}
$$

following the prescription of $\S 11.3$. Let $t>0$ and let $z_{t} \in \mathbb{P}^{2}(\mathbb{R})$ denote the point with projective coordinates $(t: t: 1)$. Only six regions $\sigma_{Q}^{P}(z)$ of the domain of integration provide a non-zero contribution to the period integral, shown below.
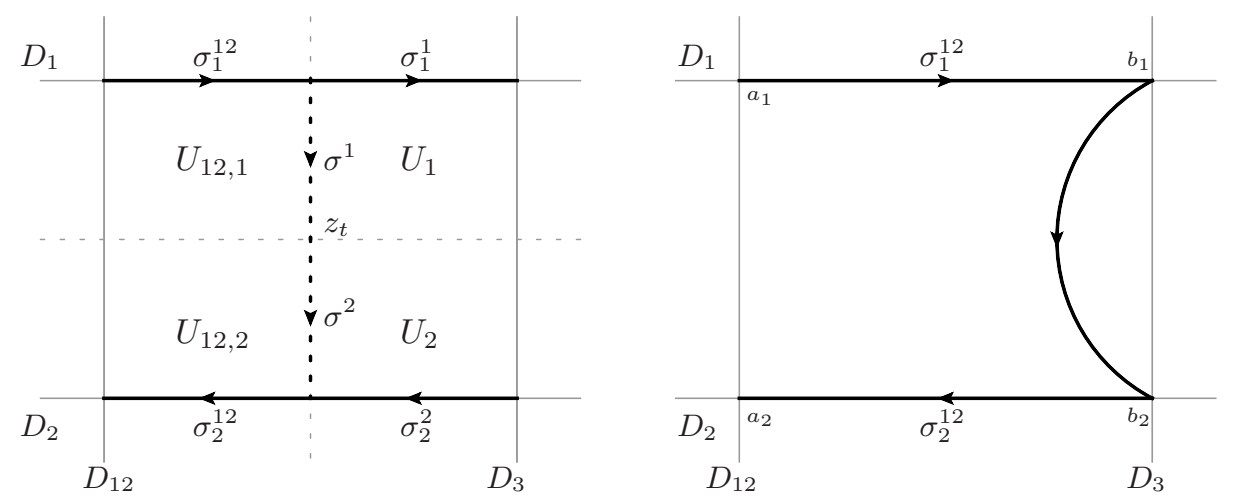

FIGURE 2. Integration of a period. Left: the non-trivial contributions to the period integral. Right: taking the limit as $t \rightarrow \infty$ and replacing the paths with tangential base points.

Here, $\sigma^{1}=\{(x: t: 1), 0 \leq x \leq t\}$ and $\sigma^{2}=\{(t: x: 1), 0 \leq x \leq t\}$. 
From the general formula (11.5) we have

$$
I=\sum_{i=1,2} \int_{\sigma_{i}^{12}(t)} \mu_{i}+\int_{\sigma_{i}^{i}(t)} \nu_{i}+\int_{\sigma^{i}(t)} \omega_{i i} .
$$

It can be computed directly, and does not depend on $t$. Instead, we shall compute it by letting $t \rightarrow \infty$ and using tangential base points.

12.0.6. Limits and tangential basepoints. Consider the following basepoints on $D_{1}$ : an ordinary base point $a_{1}=D_{1} \cap D_{12}$ (given by $\beta_{2}^{12,1}=0$ ), and a tangential base point $b_{1}$ on $D_{1}$ at the point $D_{1} \cap D_{3}$ defined by $-\partial / \partial \beta_{2}^{12,1}$. Denote the analogous basepoints on $D_{2}$ by $a_{2}, b_{2}$, as shown in the figure. Since our forms have at most logarithmic poles, we deduce on taking $t \rightarrow \infty$, that

$$
I=\int_{a_{1}}^{b_{1}} \mu_{1}+\int_{b_{2}}^{a_{2}} \mu_{2}+\int_{b_{1}}^{b_{2}} d \log \bar{Q}
$$

as shown in the previous figure. The third term is given by

$$
\lim _{t \rightarrow \infty} \log \left(\frac{\bar{Q}(t, 0)}{\bar{Q}(0, t)}\right)=\lim _{t \rightarrow \infty} \log \left(\frac{q^{2}+m_{1}^{2}(t+1)}{q^{2}+m_{2}^{2}(t+1)}\right)=\log \left(\frac{m_{1}^{2}}{m_{2}^{2}}\right) .
$$

The first term is given by

$$
\lim _{t \rightarrow-1_{\infty}} \int_{0}^{t} \frac{2 m_{2}^{2} d x}{q^{2}+m_{2}^{2}(x+1)}=2 \lim _{t \rightarrow-1_{\infty}} \log \left(\frac{q^{2}+m_{2}^{2}(t+1)}{q^{2}+m_{2}^{2}}\right)=2 \log \left(\frac{m_{2}^{2}}{q^{2}+m_{2}^{2}}\right)
$$

where $\lim _{t \rightarrow-1_{\infty}}$ denotes the regularised limit as $t \rightarrow \infty$ with respect to the tangential base point $-\partial / \partial t$ ( $m_{2}$ by $m_{1}$ in this formula and changing the sign. In total,

$$
I=2 \log \left(\frac{q^{2}+m_{1}^{2}}{q^{2}+m_{2}^{2}}\right)-\log \left(\frac{m_{1}^{2}}{m_{2}^{2}}\right) .
$$

We see that $I$ is a linear combination of limits of divergent generalised amplitudes.

On the other hand, the amplitude of the one-loop graph $G / 3$ is proportional to $\log m_{1}^{2} m_{2}^{-2}$. By the face relations, this provides another period of $G$. From the cohomology calculations, we know that the motivic periods of $\operatorname{mot}_{G}^{\prime}$ are motivic logarithms. Since these are uniquely determined by their period, we deduce the:

Corollary 12.1. The motivic periods of $\operatorname{mot}_{G}^{\prime}$ are spanned by 1 and the two motivic logarithms (on the space of generic kinematics $U_{2,2}^{\text {gen }}$ )

$$
\log ^{\mathfrak{m}}\left(\frac{m_{2}^{2}}{m_{1}^{2}}\right) \quad \text { and } \quad \log ^{\mathfrak{m}}\left(\frac{q^{2}+m_{2}^{2}}{q^{2}+m_{1}^{2}}\right) .
$$

Thus the periods of $G$ are regularised limits of linear combinations of amplitudes of the three graphs $G / 1, G / 2, G / 3$. See [19] $\S 5.3 .2$ for an interpretation of these periods in terms of hyperbolic geometry. This example illustrates how the motivic periods of small graphs can in principle be computed algorithmically. 


\section{REFERENCES}

[1] S. Abreu, R. Britto, H. Grönqvist: Cuts and coproducts of massive triangle diagrams, arXiv:1504.00206

[2] S. Abreu, R. Britto, C. Duhr, E. Gardi: From multiple unitarity cuts to the coproduct of Feynman integrals, arXiv:1401.3546

[3] L. Adams, C. Bogner, S. Weinzierl The two-loop sunrise graph in two space-time dimensions with arbitrary masses in terms of elliptic dilogarithms, arXiv:1405.5640

[4] P. Aluffi, M. Marcolli: Feynman motives and deletion-contraction relations, arXiv: $0907.3225 \mathrm{v} 1,(2009)$.

[5] Y. André: Galois theory, motives, and transcendental number theory, arXiv:0805.2569.

[6] Y. André: Ambiguity theory, old and new, arXiv:0805.2568

[7] P. Belkale, P. Brosnan: Matroids, motives, and a conjecture of Kontsevich, Duke Math. J. 116, No.1, 147-188 (2003).

[8] M. Berghoff: Wonderful renormalisation, Ph.D. thesis, Humboldt University (2015).

[9] S. Bloch: Motives associated to graphs, Japan J. Math. 2 (2007), 165196

[10] S. Bloch, H. Esnault, D. Kreimer: On motives associated to graph polynomials, Comm. Math. Phys. 267 (2006), no. 1, 181-225.

[11] S. Bloch, D. Kreimer: Feynman amplitudes and Landau singularities for 1-loop graphs, Communications in Number Theory and Physics 07/2010; 4(4), DOI:10.4310/CNTP.2010.v4.n4.a4

[12] D. Broadhurst: Multiple zeta values and modular forms in quantum field theory, in Computer algebra in Quantum Field theory (2013), pp 33-73.

[13] D. Broadhurst, D. Kreimer: Knots and numbers in $\phi^{4}$ theory to 7 loops and beyond, Int. J. Mod. Phys. C 6, 519 (1995).

[14] S. Bloch, M. Kerr, P. Vanhove: A Feynman integral via higher normal functions, arXiv:1406.2664

[15] F. Brown: Mixed Tate motives over $\mathbb{Z}$, Annals of Math., volume 175, no. 1 (2012).

[16] https://www . youtube. com/watch?v=PlJIECqRZRA

[17] F. Brown: Notes on Motivic Periods, arXiv: 1512.06410

[18] F. Brown: On the periods of some Feynman graphs, arXiv:0910.0114v1, (2009).

[19] F. Brown: Dedekind zeta motives for totally real number fields, Inventiones, Volume 194, Issue 2 (2013), Page 257-311, arXiv:0804.1654

[20] F. Brown, D. Doryn: Framings for graph hypersurfaces, arXiv:1301.3056

[21] F. Brown, D. Kreimer: Angles, scales, and parametric renormalization, Letters in Mathematical Physics, Volume 103, Issue 9 (2013), Page 933-1007, arXiv:1112.1180

[22] F. Brown, O. Schnetz: Modular forms in quantum field theory, Commun. Number Theory Phys. 7(2013) 293-325, arXiv:1304.5342

[23] F. Brown, O. Schnetz, K. Yeats: Properties of $c_{2}$ invariants of Feynman graphs, arXiv:1203.0188 (2012).

[24] P. Cartier : La folle journée, de Grothendieck à Connes et Kontsevich, Evolution des notions d'espace et de symétrie', Publications IHES (1998), archive.numdam.org/article/ PMIHES_1998__S88__23_0.pdf

[25] S. Caron-Huot, L. Dixon, A. McLeod, M. von Hippel: Bootstrapping a Five-Loop Amplitude Using Steinmann Relations, Physical Review Letters, Volume 117, Issue 24, id. 241601

[26] K. T. Chen, Iterated path integrals, Bull. Amer. Math. Soc. 83, (1977), 831-879.

[27] A. Connes, M. Marcolli: Renormalisation and motivic Galois theory, http://arxiv. org/abs/math/0409306 (2004)

[28] A. Connes, M. Marcolli: Quantum fields and motives: http://arxiv.org/abs/hep-th/ 0504085

[29] A. I. Davydychev, R. Delbourgo, A geometrical angle on Feynman integrals, J. Math. Phys. 39 (1998) 4299, arXiv:hep-th/9709216

[30] D. Doryn, On the cohomology of graph hypersurfaces associated to certain Feynman graphs, Comm. Num. Th. Phys. 4 (2010), 365-415.

[31] D. Doryn: On one example and one counterexample in counting rational points on graph hypersurfaces, arXiv:1006.3533v1 (2010).

[32] C. Dupont: Relative cohomology of bi-arrangements, arXiv:1410.6348 
[33] E. D'Hoker, M. Green, O. Gurdogan, P. Vanhove : Modular Graph Functions, arXiv: 1512.06779

[34] J. Golden, A. Goncharov, M. Spradlin, C. Vergu, A. Volovich :Motivic Amplitudes and Cluster Coordinates, http://arxiv.org/abs/1305.1617

[35] A. Goncharov, Yu. Manin: Multiple $\zeta$-motives and moduli spaces $M_{0, n}$, Compos. Math., 140(1):1-14, (2004)

[36] A. B. Goncharov, M. Spradlin, C. Vergu, A. Volovich: Classical Polylogarithms for Amplitudes and Wilson Loops, Phys. Rev. Lett. 105, 151605 (2010)

[37] A. Grothendieck: On the de Rham cohomology of algebraic varieties, Publications mathmatiques de II.H.E.S., tome 29 (1966), p. 95-103

[38] R. Kaufmann, B. Ward : Feynman categories, arXiv:1312.126

[39] G. Kirchhoff: Ueber die Auflösung der Gleichungen, auf welche man bei der Untersuchung der linearen Vertheilung galvanischer Ströme gehührt wird, Annalen der Physik und Chemie 72 no. 12 (1847), 497-508.

[40] M. Kontsevich, D. Zagier: Periods, Mathematics unlimited-2001 and beyond, 771-808, Springer, Berlin, (2001)

[41] D. Kreimer, M. Sars, W. D. van Suijlekom: Quantization of gauge fields, graph polynomials and graph homology, Annals of Physics 08/2012; 336, DOI:10.1016/j.aop.2013.04.019

[42] S. Müller-Stach, S. Weinzierl, R. Zayadeh: A second-order differential equation for the two-loop sunrise graph with arbitrary masses, arXiv:1112.4360

[43] B.G. Nickel: Evaluation of Simple Feynman Graphs, J.Math.Phys.19:542-548,1978

[44] E. Panzer : Feynman integrals and hyperlogarithms, Ph.D thesis, arXiv:1506.07243

[45] E. Panzer: Algorithms for the symbolic integration of hyperlogarithms with applications to Feynman integrals, Computer Physics Communications, 188 (2015), pp. 148-166

[46] E. Panzer, O. Schnetz: The Galois action on $\phi^{4}$ periods, preprint.

[47] O. Schlotterer, S. Stieberger: Motivic Multiple Zeta Values and Superstring Amplitudes, arXiv:1205.1516

[48] O. Schnetz: Quantum periods: A census of $\phi^{4}$ transcendentals, Jour. Numb. Theory and Phys. 4 no. 1 (2010), 1-48.

[49] O. Schnetz : The geometry of one-loop amplitudes, arXiv:1010.5334

[50] V. Smirnov: Evaluating Feynman integrals, Springer Tracts in Modern Physics 211. Berlin: Springer. ix, 247 p. (2004). [ISBN 3-540-23933-2]

[51] E. Speer: Ultraviolet and infrared singularity structure of generic Feynman amplitudes, Ann. Inst. H. Poincar Sect. A (N.S.) 23 (1975), no. 1, 1-21.

[52] E. Speer, M. Westwater: Generic Feynman amplitudes, Ann. Inst. H. Poincar Sect. A (N.S.) 14 (1971), 1-55.

[53] S. Stieberger, T. Taylor: Closed string amplitudes as single-valued open string amplitudes, Nuclear Phys. B 881 (2014), 269-287.

[54] S. Weinberg: High-Energy Behavior in Quantum Field Theory, Phys. Rev. 118, no. 3 838-849 (1960).

[55] F. Zerbini: Single-valued multiple zeta values in genus 1 superstring amplitudes, arXiv: 1512.05689 